\title{
S-WAVE VELOCITY ESTIMATION USING CONVERTED-WAVE VSP DATA
}

\author{
A Thesis \\ Presented to \\ the Faculty of the Department of Earth and Atmospheric Sciences \\ University of Houston \\ In Partial Fulfillment \\ of the Requirements for the Degree \\ Master of Science \\ By \\ Minyu Zhang \\ December 2013
}




\section{S-WAVE VELOCITY ESTIMATION USING CONVERTED-WAVE VSP DATA}

Minyu Zhang

APPROVED:

Dr. Robert Stewart, Committee Chair

Department of Earth and Atmospheric Sciences

Dr, Aibing Li, Committee member

Department of Earth and Atmospheric Sciences

Dr. Chaoshun $\mathrm{Hu}$, Committee member Chevron

Dean

College of Natural Sciences and Mathematics 


\section{Acknowledgements}

First and foremost, I would like to thank my advisor, Dr. Robert Stewart, for all the exciting moments I shared with him in our Allied Geophysics Laboratory (AGL) group. I have profoundly benefited from my association with the group over the past three years. Without his guidance and supervision I could not survive as a geophysicist. I am also fortunate to have Dr. Aibing Li and Dr. Chaoshun Hu as my committee members. I truly acknowledge their valuable time and patience.

The strong sense of community within the AGL group has been an important part of my time at UH. I would like to express my appreciation to the group members Li Chang, Yue Du, Long Huang, Eray Kocel, Jiannan Wang, Anoop William, and Jingjing Zong for their kind help.

I started the internship at Halliburton in the summer of 2013. Because of that, I was given a chance to gain valuable industry experience. I owe my deepest gratitude to Mr. Dan Quinn and Mr. Malcolm Hollingsworth for bringing me there. Advice and encouragement from my internship mentor, Dr. Ran Zhou, were always important guiding lights towards my personal and professional development. I had a fruitful summer there and I would like to express my appreciation to them.

Finally, I come to the most personal source of gratitude. My sincere thanks to my husband, Dr. Ge Zhan. This study is the result of his support, encouragement, help and love. I thank him for giving me wholehearted love during my studies. 


\title{
S-WAVE VELOCITY ESTIMATION USING CONVERTED-WAVE VSP DATA
}

\author{
An Abstract of a Thesis \\ Presented to \\ the Faculty of the Department of Earth and Atmospheric Sciences \\ University of Houston
}

In Partial Fulfillment

of the Requirements for the Degree

Master of Science

By

Minyu Zhang

December 2013 


\section{Abstract}

One of the key benefits of zero-offset vertical seismic profile (VSP) is to provide accurate velocity information in the vicinity of boreholes. For 3-component VSP, we usually pick the first break times of direct downgoing S-wave to estimate the S-wave velocity. However, the direct downgoing S-wave arrivals may be too weak to pick precisely. To mitigate this disadvantage, I present a new S-wave velocity estimation method using converted (P-to-S) waves in VSP data. Traveltimes of the strongest converted PS-wave are used to build an S-wave velocity model.

Two synthetic traveltime datasets are generated and used to test the method. The first one, without noise, is used to investigate the sensitivities of results to different initial models. The inversion results are slightly different: the RMS error between the true velocity and the inverted velocity varies from $1 \mathrm{~m} / \mathrm{s}$ to $10 \mathrm{~m} / \mathrm{s}$ for different initial models. The second test, with random noise, explores the sensitivities of results to errors in picked traveltimes. Results show that the RMS error of output velocity varies from $2 \mathrm{~m} / \mathrm{s}$ to $92 \mathrm{~m} / \mathrm{s}$ when the RMS error of input traveltimes ranges from $0.1 \mathrm{~ms}$ to $8 \mathrm{~ms}$.

In addition to the synthetic tests, real 3-component zero-offset VSP and offset VSP datasets from the Huabei oil field, China are examined using the proposed method. The VSP survey used a dynamite source and 3-component receivers, with the receiver depth from $360 \mathrm{~m}$ to $2020 \mathrm{~m}$. The inversion results show that, from $360 m$ to $900 m$ depths, the S-wave velocity derived from offset VSP fits quite well

with that obtained from zero-offset VSP. The $V_{p} / V_{s}$ is approximated 2.8 and the lithology is mudstone with sandstone. From $910 m$ to $2020 m$, the S-wave velocity 
derived from offset VSP is close to that from zero-offset VSP. The $V_{p} / V_{s}$ is around 1.7 and the lithology is primarily limestone and dolomite. 


\section{Contents}

1 Introduction 1

1.1 Motivation . . . . . . . . . . . . . . . . . . 1

1.2 Thesis overview $\ldots \ldots \ldots \ldots \ldots \ldots \ldots \ldots \ldots \ldots$

2 Method 4

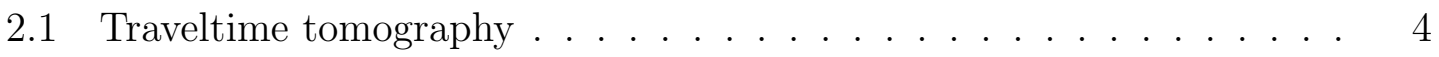

2.2 Converted PS-wave traveltime calculation $\ldots \ldots \ldots \ldots$

2.3 S-wave velocity estimation $\ldots \ldots \ldots \ldots \ldots$

2.3.1 Initial S-wave velocity building . . . . . . . . . . . . 12

2.3.2 PS-wave ray tracing . . . . . . . . . . . . . . . . 12

2.3 .3 S-wave velocity inversion . . . . . . . . . . . 13

3 Synthetic data test $\quad 16$

3.1 Synthetic model I . . . . . . . . . . . . . . . . . . . . . . 16

3.2 Synthetic model II . . . . . . . . . . . . . . . . . . . . . 20

3.3 Summary . . . . . . . . . . . . . . . . . . 24

4 Field data test $\quad 25$

4.1 Introduction . . . . . . . . . . . . . . . 25

$4.2 \quad$ VSP acquisition parameters $\ldots \ldots \ldots \ldots \ldots \ldots$

4.3 Raw data analysis . . . . . . . . . . . . . . . . . 31 
$4.43 \mathrm{C}$ VSP data processing . . . . . . . . . . . . . . . 31

4.4.1 Zero-offset VSP . . . . . . . . . . . . . . . 34

4.4 .2 Offset VSP . . . . . . . . . . . . . . . . . 47

4.5 S-wave velocity estimation . . . . . . . . . . . . . . . . . . . . . 49

4.6 VSP-CDP mapping and VSP-CCP mapping . . . . . . . . . . . 57

4.7 Summary ........................ 60

5 Conclusion $\quad 64$

5.1 Summary .............................. 64

5.2 Future research work . . . . . . . . . . . . . . . 65

$\begin{array}{ll}\text { References } & 66\end{array}$

Appendix A Groundroll analysis using VSP-type processing $\quad 68$

A.1 Workflow of groundroll processing . . . . . . . . . . . . . . . . . 69

A.1.1 Raw data analysis . . . . . . . . . . . . . . 69

A.1.2 Traveltime picking and velocity analysis . . . . . . . . . . 71

A.1.3 Wavefield separation ................ 73

A.1.4 Deconvolution . . . . . . . . . . . . . . . . . 74

A.1.5 Corridor mute and corridor stack . . . . . . . . . . . 76

A.1.6 Bin stack and F-X transform . . . . . . . . . . . . . . 76 


\section{List of Figures}

1.1 Three-component $\left(Z, H_{1}, H_{2}\right)$ VSP field data after rotation. (a) shows the zero-offset VSP where the direct S-waves are weak. (b) is the offset VSP which shows strong converted PS-waves. . . . . . . . . . . 2

2.1 Workflow of traveltime tomography. . . . . . . . . . . . . . 5

2.2 Ray path of converted PS-wave overlaid on the velocity model. . . . . 6

2.3 Converted PS-wave ray paths overlaid on the velocity model. . . . . . 9

2.4 Illustration of traveltime calculation in terms of the ray path. . . . . 10

2.5 Diagram show of the shooting method. . . . . . . . . . . . . . . 13

2.6 Workflow of converted wave traveltime inversion. . . . . . . . . . . . 14

3.1 Synthetic model I and PS-wave ray paths of a VSP shot. . . . . . . . 17

3.2 Inversion result using initial guess $1 . \quad \ldots \ldots \ldots$

3.3 Differences between true $V_{s}$ and inverted $V_{s}$ using initial guess 1. . . . 19

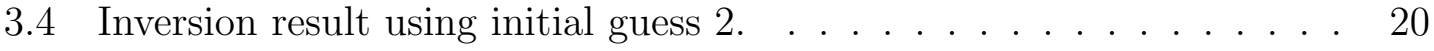

3.5 Differences between true $V_{s}$ and inverted $V_{s}$ using initial guess 2. . . 20

3.6 Synthetic model II and PS-wave ray paths of a VSP shot. . . . . . . . 21

3.7 PS-wave seismogram obtained from elastic finite-difference modeling with random noise. . . . . . . . . . . . . . . . . . . . . . . . 22

3.8 Inverted result using calculated PS-wave traveltimes. . . . . . . . . . 24

3.9 Inverted result using picked PS-wave traveltimes with errors. . . . . . 24 
4.1 Geographic location of the Huabei oil field. . . . . . . . . . . . . 26

4.2 (a) A surface seismic section of the studying area. (b) Geological

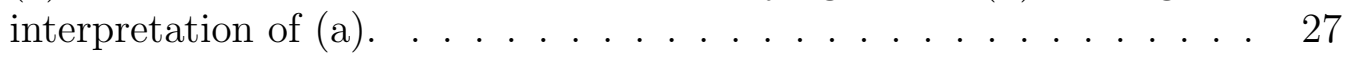

4.3 Vertical component of the (a) zero-offset VSP and the (b) offset VSP. 28

4.4 Density, GR and AC logs of this well. . . . . . . . . . . . . . . . . . . 29

4.5 Shots and receivers distribution of the VSP survey. . . . . . . . . . . 30

4.6 Raw zero-offset VSP data (a) before and (b) after rotation. . . . . . . 32

4.7 Raw offset VSP data (a) before and (b) after rotation. . . . . . . . . 33

4.8 3C zero-offset VSP raw data. . . . . . . . . . . . . . . . . . . . . . 34

4.9 Separated vertical (left) and two horizontal (middle and right) components of the zero-offset VSP. . . . . . . . . . . . . . . . . 35

4.10 Vertical component of the zero-offset VSP data. The red line is the picked first break times of direct P-wave. . . . . . . . . . . . . . . 36

$4.11 \mathrm{P}$-wave interval velocity estimated from direct $\mathrm{P}$-wave traveltimes. . . 36

4.12 The vertical component before (right) and after (left) true amplitude recovery. . . . . . . . . . . . . . . . . . 37

4.13 The vertical component before (left) and after (right) applying the bandpass filter. . . . . . . . . . . . . . . . . 38

4.14 Wavefield separation results using the FK filter. (a) is the separated downgoing waves and (b) is the upgoing waves. . . . . . . . . . . . . 40

4.15 Result after applying the median filter. (a) shows all waves; (b) is the flattened waves; (c) is separated downgoing waves; and (d) is the

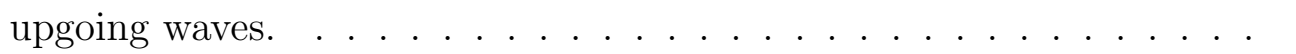

4.16 (a) Flattened downgoing waves (left) and upgoing waves (right). (b) Deconvolved downgoing waves (left) and upgoing waves (right). . . . 42

4.17 Corridor mute (left) and corridor stack (right) of the zero-offset VSP. 44

4.18 Interval velocity (pink) of zero-offset VSP compared with the acoustic velocity (blue). . . . . . . . . . . . . . . . . . 45

4.19 The VSP corridor stack projected on the surface seismic profile. . . . 46

$4.203 \mathrm{C}$ offset VSP before and after rotation. . . . . . . . . . . . . . . 48 
4.21 (a) Separated upgoing P-waves. (b) Separated upgoing PS-waves. . . 50

4.22 Upgoing P-waves (a) before and (b) after deconvolution. . . . . . . . 51

4.23 Upgoing PS-waves (a) before and (b) after deconvolution. . . . . . . . 52

4.24 Picked converted PS-wave traveltimes (a) for depth $360 m-910 m$ and (b) for all depths. . . . . . . . . . . . . . . . . 54

4.25 P-wave velocity (blue curve) and initial S-wave velocity (red curve). . 55

4.26 Ray tracing for converted PS-waves (a) for depth $360 m-910 m$ and (b) for all depths. . . . . . . . . . . . . . . . . 56 56

4.27 Inverted S-wave velocity (a) for depth $360 m-910 m$ and (b) for all depths. . . . . . . . . . . . . . . . . . 58

4.28 The ratio of $V_{p} / V_{s} \ldots \ldots \ldots \ldots \ldots \ldots$

4.29 Illustration of $\mathrm{P}$-wave reflection point and PS-wave conversion point. $\quad 60$

4.30 VSP-CDP map of P-waves versus VSP-CCP map of converted waves. 62

4.31 L-plot consisting of various P-wave and PS-wave sections. . . . . . . . 63

A.1 Physical model that includes two materials: Plexiglas and aluminum. $\quad 69$

A.2 (a) Surface geometry used for modeling the groundroll. (b) Virtual zero-offset VSP geometry transferred from the actual surface geometry. 70

A.3 Workflow of processing groundroll as a virtual zero-offset VSP. . . . . 71

A.4 (a) Raw groundroll data transferred to virtual zero-offset VSP data.

(b) Picked first break times of groundroll and a zoom view of it. . . . 72

A.5 Average velocity (left) versus interval velocity (right) . . . . . . . 73

A.6 Downgoing and upgoing waves separated by FK filtering. . . . . . . . 74

A.7 Separated downgoing and upgoing waves via the use of median filter. $\quad 75$

A.8 (a) Downgoing groundroll before and after deconvolution. (b) Upgoing groundroll before and after deconvolution. . . . . . . . . . . . 77

A.9 Corridor mute (left) and corridor stack (right) . . . . . . . . 78

A.10 Bin stack and its FX transform. . . . . . . . . . . . . . . . . . 79 


\section{List of Tables}

3.1 Properties of synthetic model I. . . . . . . . . . . . . . . . 18

3.2 Traveltimes associated with synthetic model II. . . . . . . . . . . . . 23

4.1 Generalized stratigraphy of the studying area. . . . . . . . . . . . 29

4.2 Acquisition parameters of 3C zero-offset and offset VSPs. . . . . . . . 30 


\section{Chapter 1}

\section{Introduction}

\subsection{Motivation}

The estimation of accurate $\mathrm{P}$-wave and S-wave velocities is a crucial step in seismic imaging (Stewart et al., 2002, 2003). There are many approaches for acquiring P-wave velocity from zero-offset vertical seismic profile (VSP). However, accurate estimation of S-wave velocity from zero-offset VSP is quite difficult because of technology and cost limitations (Gaiser et al., 1982; Li et al., 2005).

Figure 1.1 shows a three-component (3C) VSP field data generated from a dynamite source. On the zero-offset VSP (Figure 1.1a), the downgoing direct S-waves are hard to see, thus it is difficult to use them for S-wave velocity estimation. Fortunately, the P-to-S (PS) converted waves on the offset VSP (Figure 1.1b) are strong

enough, such that they could be possibly used to acquire S-wave velocity (Luo et al., 
2006; Hardage et al., 2011).

To overcome the inability to estimate S-wave velocity using VSP S-waves, I present a new S-wave velocity estimation method using VSP converted waves. Instead of picking the direct downgoing S-wave first breaks, the converted PS-wave traveltimes are picked and used to derive the S-wave velocity.

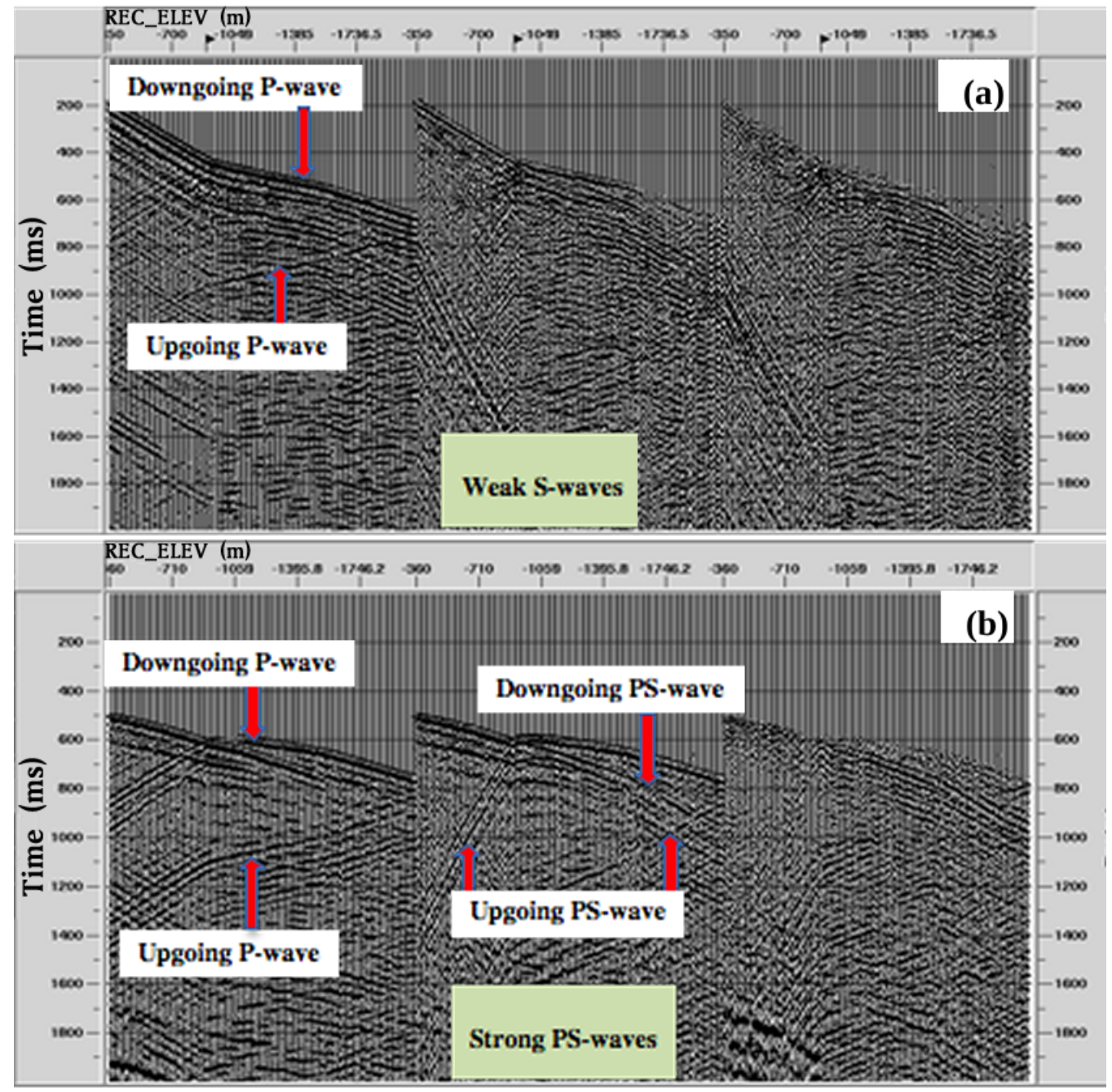

Figure 1.1: Three-component $\left(Z, H_{1}, H_{2}\right)$ VSP field data after rotation. (a) shows the zero-offset VSP where the direct S-waves are weak. (b) is the offset VSP which shows strong converted PS-waves. 


\subsection{Thesis overview}

There are five chapters in this thesis, and they are summarized below.

Chapter 1 starts with the motivation, followed by the overview of the thesis.

Chapter 2 begins with the introduction of background knowledge about traveltime tomography, followed by a discussion on how to calculate converted wave traveltimes. Two equations for computing PS-wave traveltimes are formulated. After that, details on how to estimate S-wave velocity using converted wave traveltimes are presented.

Chapter 3 shows numerical tests with synthetic VSP data. Two synthetic models are used to demonstrate the effectiveness of this method.

Chapter 4 shows numerical tests with field VSP data. It starts with an introduction of the 3C VSP field data. After that, the VSP data processing workflow is given via the demonstration with this field data. The workflow includes the processing of P-wave, converted wave, zero-offset VSP and offset VSP. Finally, the S-wave velocity associated with this data is inverted using the proposed method.

Chapter 5 summarizes the thesis work. 


\section{Chapter 2}

\section{Method}

This chapter presents the theory of the method. First, the traveltime tomography is briefly introduced. Then I show how to calculate converted wave traveltimes, where two equations are formulated to compute traveltimes of upgoing and downgoing PSwaves. Finally, the workflow of the S-wave velocity estimation method is discussed.

\subsection{Traveltime tomography}

Given an initial guess, traveltime tomography starts with forward modeling by ray tracing and computes the associated traveltimes. The tomography must then iterate to converge to the best estimate of the true model by minimizing the differences between the observed traveltimes and the modeled ones. Figure 2.1 shows the basic workflow of traveltime tomography. 


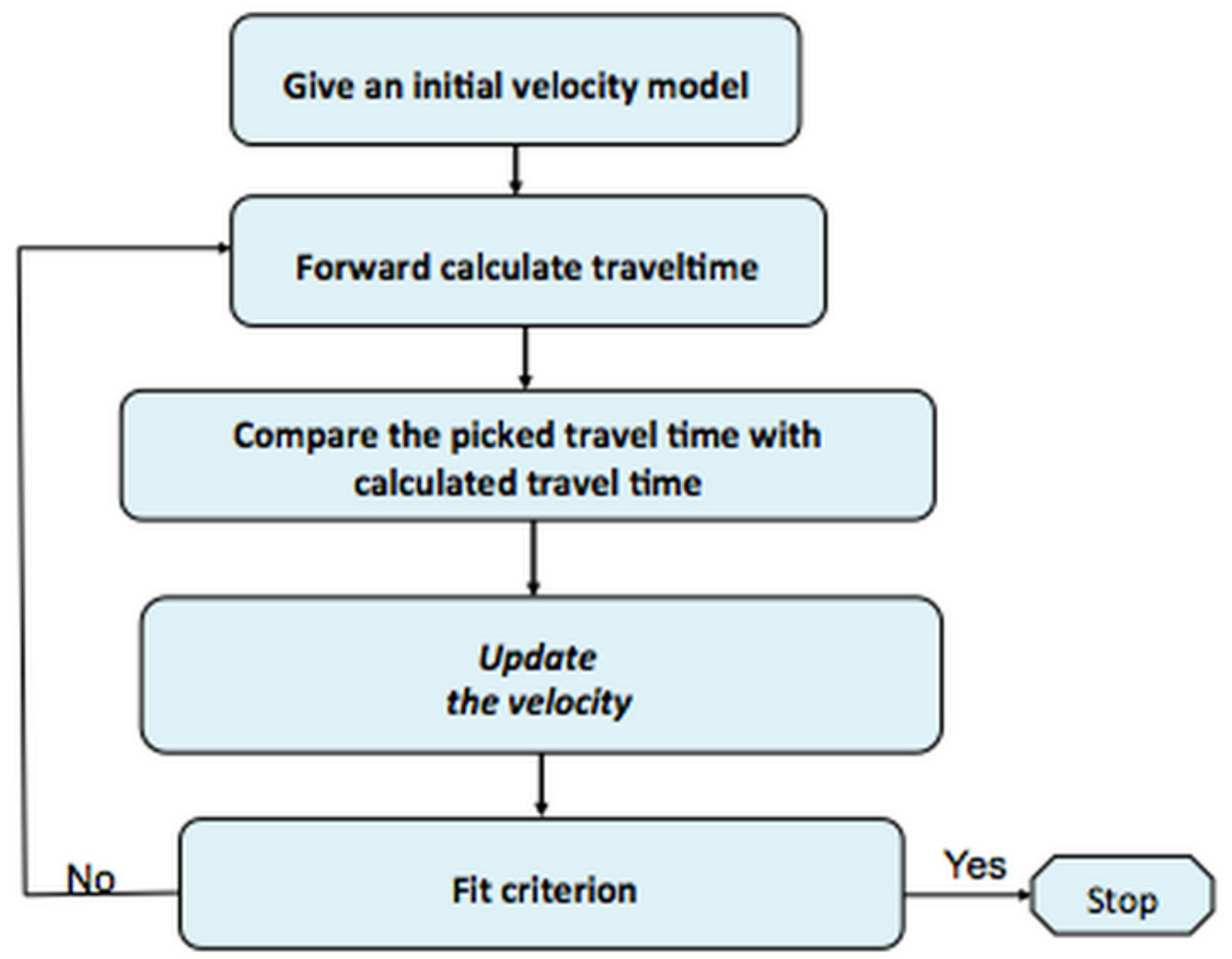

Figure 2.1: Workflow of traveltime tomography.

Traveltime tomography computes traveltimes iteratively, and traveltime differences between the modeled ones and the observed ones are used to update the velocity (Jones, 2010). Therefore, traveltime calculation is a critical step for traveltime tomography.

\subsection{Converted PS-wave traveltime calculation}

In this section, I show how to compute converted wave traveltimes according to the PS-wave ray path. 
Let's first consider a simple layer model as shown in Figure 2.2. The red point and blue point are source location and receiver location, respectively. The black line is the ray path of downgoing $\mathrm{P}$-wave, and the red line is that of upgoing S-wave.

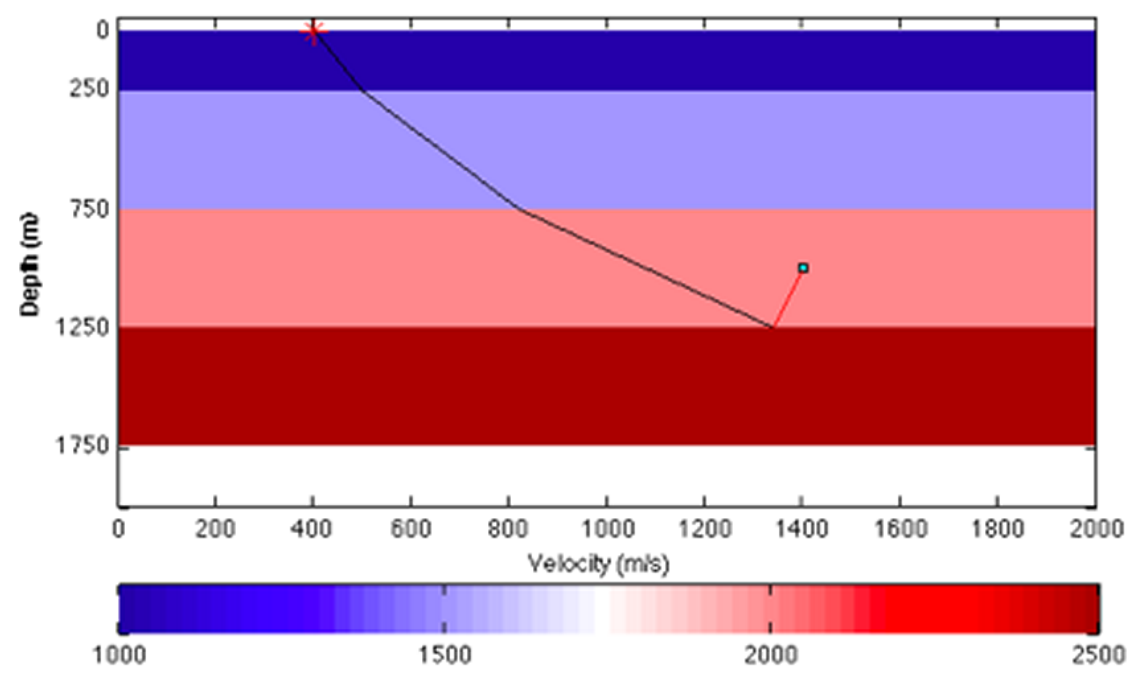

Figure 2.2: Ray path of converted PS-wave overlaid on the velocity model.

The traveltime $t$ for this ray path is the traveltime sum of downgoing $\mathrm{P}$-wave and upgoing PS-wave

$$
t=\left(\frac{l_{1}}{v_{1}}+\frac{l_{2}}{v_{2}}+\frac{l_{3}}{v_{3}}\right)+\frac{l_{3}^{s}}{v_{3}^{s}},
$$

where $l_{i}$ and $v_{i}$ are the ray length and interval velocity of the $i t h$ layer, respectively. For real case, we have more than one traveltime, so that the above expression can be generalized into a matrix notation

$$
T=L^{p} S^{p}+L^{s} S^{s}
$$

where $S^{p}$ and $S^{s}$ are the reciprocals of $\mathrm{P}$-wave velocity and $\mathrm{S}$-ware velocity, respectively; $L=\left[l_{1}, l_{2}, \ldots, l_{n}\right]$, and $T=\left[t_{1}, t_{2}, \ldots, t_{n}\right]^{T}$. 
The matrix expression for computing PS-wave traveltimes can be formulated by expanding equation 2.2. Suppose that the P-wave travels downward from the 1 st to the nth layer, and the S-wave goes upward from the $n$th to the 1 st layer, the following equation can be used to calculate the traveltimes of upgoing PS-wave

$$
\left(\begin{array}{ccccc}
l_{1} & l_{2} & l_{3} \ldots l_{n-1} & l_{n} \\
l_{1} & l_{2} & l_{3} \ldots l_{n-1} & l_{n} \\
& \vdots & \ddots & \vdots \\
l_{1} & l_{2} & l_{3} \ldots l_{n-1} & l_{n} \\
l_{1} & l_{2} & l_{3} \ldots l_{n-1} & l_{n}
\end{array}\right)\left(\begin{array}{c}
s_{1}^{p} \\
s_{2}^{p} \\
\vdots \\
s_{n-1}^{p} \\
s_{n}^{p}
\end{array}\right)+\left(\begin{array}{cccc}
l_{1}^{s} & l_{2}^{s} & l_{3}^{s} \ldots l_{n-1}^{s} & l_{n}^{s} \\
0 & l_{2}^{s} & l_{3}^{s} \ldots l_{n-1}^{s} & l_{n}^{s} \\
& \vdots & \ddots & \vdots \\
0 & 0 & 0 \ldots l_{n-1}^{s} & l_{n}^{s} \\
0 & 0 & 0 \ldots 0 & l_{n}^{s}
\end{array}\right)\left(\begin{array}{c}
s_{1}^{s} \\
s_{2}^{s} \\
\vdots \\
s_{n-1}^{s} \\
s_{n}^{s}
\end{array}\right)=\left(\begin{array}{c}
t_{1}^{p s} \\
t_{2}^{p s} \\
\vdots \\
t_{n-1}^{p s} \\
t_{n}^{p s}
\end{array}\right),
$$

where $l_{i}$ is the $\mathrm{P}$-wave ray length in the $i$ th layer; $l_{i}^{s}$ is the $\mathrm{S}$-wave ray length in the ith layer; $s_{i}^{p}$ and $s_{i}^{s}$ are the $\mathrm{P}$-wave and S-wave slowness of the $i t h$ layer, respectively; and $t_{i}^{p s}$ is the calculated PS-wave traveltime in the $i t h$ layer. In the above expression, the first term is

$$
\left(\begin{array}{cccc}
l_{1} & l_{2} & l_{3} \ldots l_{n-1} & l_{n} \\
l_{1} & l_{2} & l_{3} \ldots l_{n-1} & l_{n} \\
& \vdots & \ddots & \vdots \\
l_{1} & l_{2} & l_{3} \ldots l_{n-1} & l_{n} \\
l_{1} & l_{2} & l_{3} \ldots l_{n-1} & l_{n}
\end{array}\right)\left(\begin{array}{c}
s_{1}^{p} \\
s_{2}^{p} \\
\vdots \\
s_{n-1}^{p} \\
s_{n}^{p}
\end{array}\right)=\left(\begin{array}{c}
t_{1}^{p} \\
t_{2}^{p} \\
\vdots \\
t_{n-1}^{p} \\
t_{n}^{p}
\end{array}\right)
$$

which only involves the $\mathrm{P}$-wave traveltime calculation. We can easily get the direct $\mathrm{P}$-wave traveltimes via first break time pickings. And the $\mathrm{P}$-wave velocity can then 
be obtained by $\mathrm{P}$-wave traveltime tomography. The second term is

$$
\left(\begin{array}{cccc}
l_{1}^{s} & l_{2}^{s} & l_{3}^{s} \ldots l_{n-1}^{s} & l_{n}^{s} \\
0 & l_{2}^{s} & l_{3}^{s} \ldots l_{n-1}^{s} & l_{n}^{s} \\
& \vdots & \ddots & \vdots \\
0 & 0 & 0 \ldots l_{n-1}^{s} & l_{n}^{s} \\
0 & 0 & 0 \ldots 0 & l_{n}^{s}
\end{array}\right)\left(\begin{array}{c}
s_{1}^{s} \\
s_{2}^{s} \\
\vdots \\
s_{n-1}^{s} \\
s_{n}^{s}
\end{array}\right)=\left(\begin{array}{c}
t_{1}^{s} \\
t_{2}^{s} \\
\vdots \\
t_{n-1}^{s} \\
t_{n}^{s}
\end{array}\right)
$$

which only relates to the S-wave traveltime calculation.

In order to help to understand this equation, I use a simple layer velocity model for explanation. Figure 2.3 shows a layered velocity model overlaid by PS-wave ray paths for illustration. The red point is the shot location and the green squares are receiver locations. The black line segments are ray paths of downgoing P-wave, while the red lines are ray paths of upgoing S-wave.

Equation 2.3 is used to compute upgoing converted PS-wave traveltime. Converted wave traveltime of the first receiver is calculated as following

$$
\left(l_{1}^{p} s_{1}^{p}+l_{1}^{p} s_{1}^{p}+\ldots+l_{17}^{p} s_{17}^{p}+l_{18}^{p} s_{18}^{p}\right)+\left(l_{1}^{s} s_{1}^{s}+l_{1}^{s} s_{1}^{s}+\ldots+l_{17}^{s} s_{17}^{s}+l_{18}^{s} s_{18}^{s}\right)=t_{1}^{p s}
$$

For the last receiver, the traveltime is calculated as below

$$
\left(l_{1}^{p} s_{1}^{p}+l_{1}^{p} s_{1}^{p}+\ldots+l_{17}^{p} s_{17}^{p}+l_{18}^{p} s_{18}^{p}\right)+l_{18}^{2} s_{18}^{s}=t_{18}^{p s}
$$




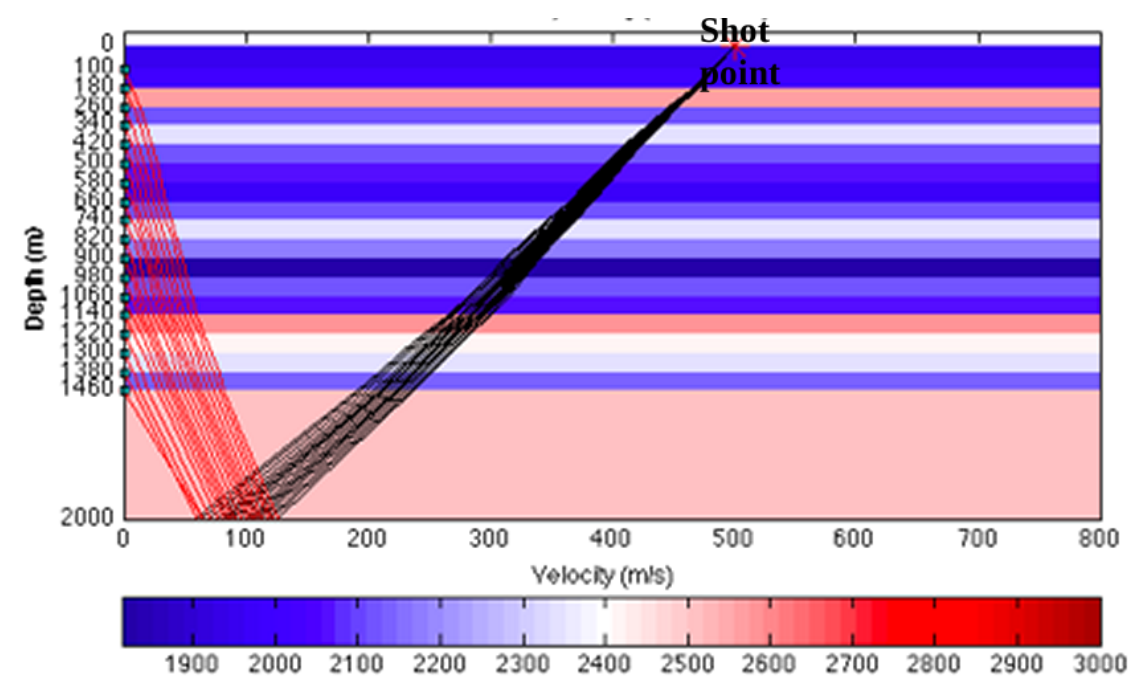

Figure 2.3: Converted PS-wave ray paths overlaid on the velocity model.

If both $\mathrm{P}$-wave and converted S-wave are downgoing, the following matrix expression is used instead of equation 2.3 to calculate the PS-wave traveltimes

$$
\left(\begin{array}{ccccc}
l_{1} & l_{2} & l_{3} \ldots l_{n-1} & l_{n} \\
l_{1} & l_{2} & l_{3} \ldots l_{n-1} & l_{n} \\
& \vdots & \ddots & \vdots \\
l_{1} & l_{2} & l_{3} \ldots l_{n-1} & l_{n} \\
l_{1} & l_{2} & l_{3} \ldots l_{n-1} & l_{n}
\end{array}\right)\left(\begin{array}{c}
s_{1}^{p} \\
s_{2}^{p} \\
\vdots \\
s_{n-1}^{p} \\
s_{n}^{p}
\end{array}\right)+\left(\begin{array}{cccc}
l_{1}^{s} & 0 & 0 \ldots 0 & 0 \\
l_{1}^{s} & l_{2}^{s} & 0 \ldots 0 & 0 \\
& \vdots & \ddots & \vdots \\
l_{1}^{s} & l_{2}^{s} & l_{3}^{s} \ldots l_{n-1}^{s} & 0 \\
l_{1}^{s} & l_{2}^{s} & l_{3}^{s} \ldots l_{n-1}^{s} & l_{n}^{s}
\end{array}\right)\left(\begin{array}{c}
s_{1}^{s} \\
s_{2}^{s} \\
\vdots \\
s_{n-1}^{s} \\
s_{n}^{s}
\end{array}\right)=\left(\begin{array}{c}
t_{1}^{p s} \\
t_{2}^{p s} \\
\vdots \\
t_{n-1}^{p s} \\
t_{n}^{p s}
\end{array}\right) .
$$

The traveltime calculation is mathematically discussed above. Next, I show how to calculate PS-wave traveltime practically. Let's consider a layer model shown in Figure 2.4. Here, $v$ and $\Delta x$ are velocity and offset of each layer, respectively; $\Delta z$ is the thickness, $\theta$ is the incidence angle, and $p$ is ray parameter

$$
p=\frac{\sin \theta}{v} .
$$




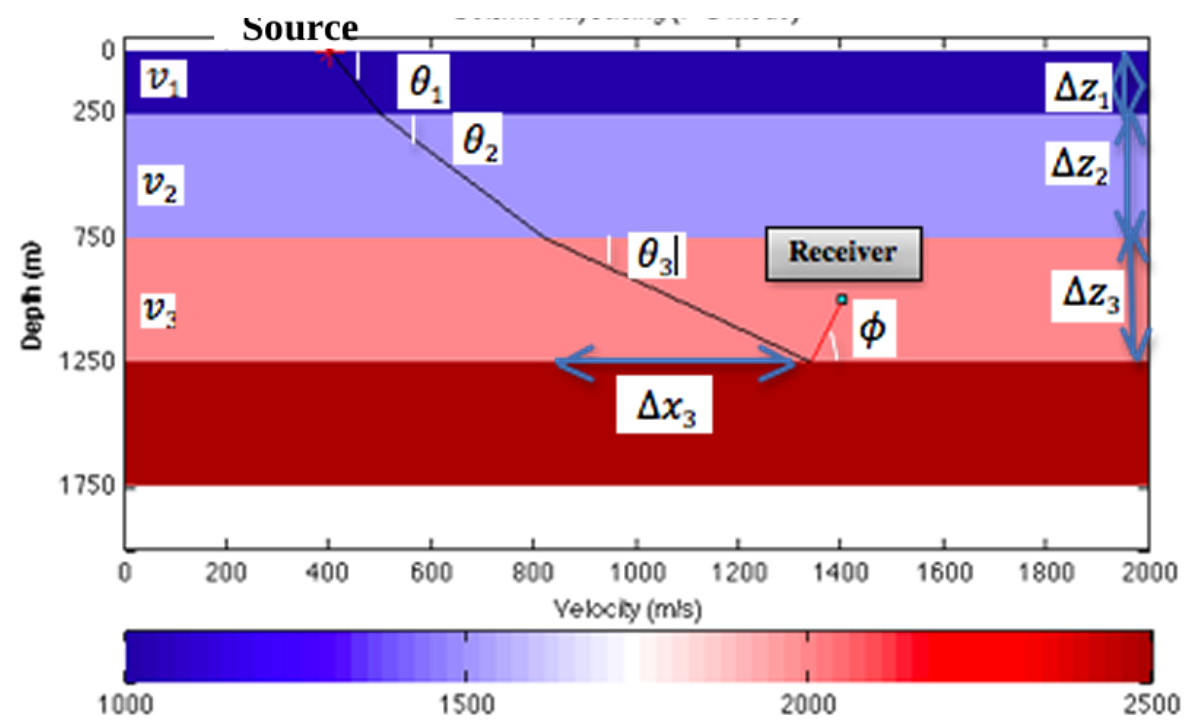

Figure 2.4: Illustration of traveltime calculation in terms of the ray path.

Because

$$
\frac{\Delta x}{\Delta d}=\sin \theta=p v
$$

we have

$$
\frac{\Delta x}{\Delta d}=\cos \theta=u v=\sqrt{1-p^{2} v^{2}},
$$

so that

$$
\frac{\Delta x}{\Delta z}=\frac{\Delta x}{\Delta d} \cdot \frac{\Delta d}{\Delta z}=\frac{p v}{\sqrt{1-p^{2} v^{2}}} .
$$

We can calculate travel distance as well as traveltime in each layer

$$
\Delta x=\frac{p v}{\sqrt{1-p^{2} v^{2}}} \Delta ; \quad \Delta t=\frac{1}{v \sqrt{1-p^{2} v^{2}}} \Delta z,
$$

because the ray path intersecting at the interface satisfies Snell's law

$$
\frac{\sin \theta_{i}}{v_{i}}=\frac{\sin \theta_{i+1}}{v_{i+1}} .
$$

For PS-wave ray path, Snells law can also describe the geometry

$$
\frac{\sin \theta}{v_{p}}=\frac{\sin \phi}{v_{s}},
$$


where $\theta$ and $\phi$ are the $\mathrm{P}$-wave and S-wave angles of incidence and reflection, respectively; and $v_{p}$ and $v_{s}$ are the corresponding $\mathrm{P}$-wave velocity and S-wave wave velocity.

We can easily calculate the traveltime. The traveltime of this ray path is

$$
t^{p s}=\sum_{j=1}^{3} \frac{\Delta z_{j}}{v_{p_{j}}} \sqrt{1-p^{2} v_{p_{j}}^{2}}+\frac{\Delta z_{3}}{v_{s_{3}} \sqrt{1-p^{\prime 2} v_{s_{3}}^{2}}},
$$

where $\Delta z_{j}$ is the thickness of the $j t h$ layer; $v_{p_{j}}$ and $v_{s_{j}}$ are $\mathrm{P}$-wave velocity and S-wave velocity in the $j$ th layer, and $p^{\prime}$ is ray parameter of S-wave.

A Matlab code segment for traveltime calculation is given below.

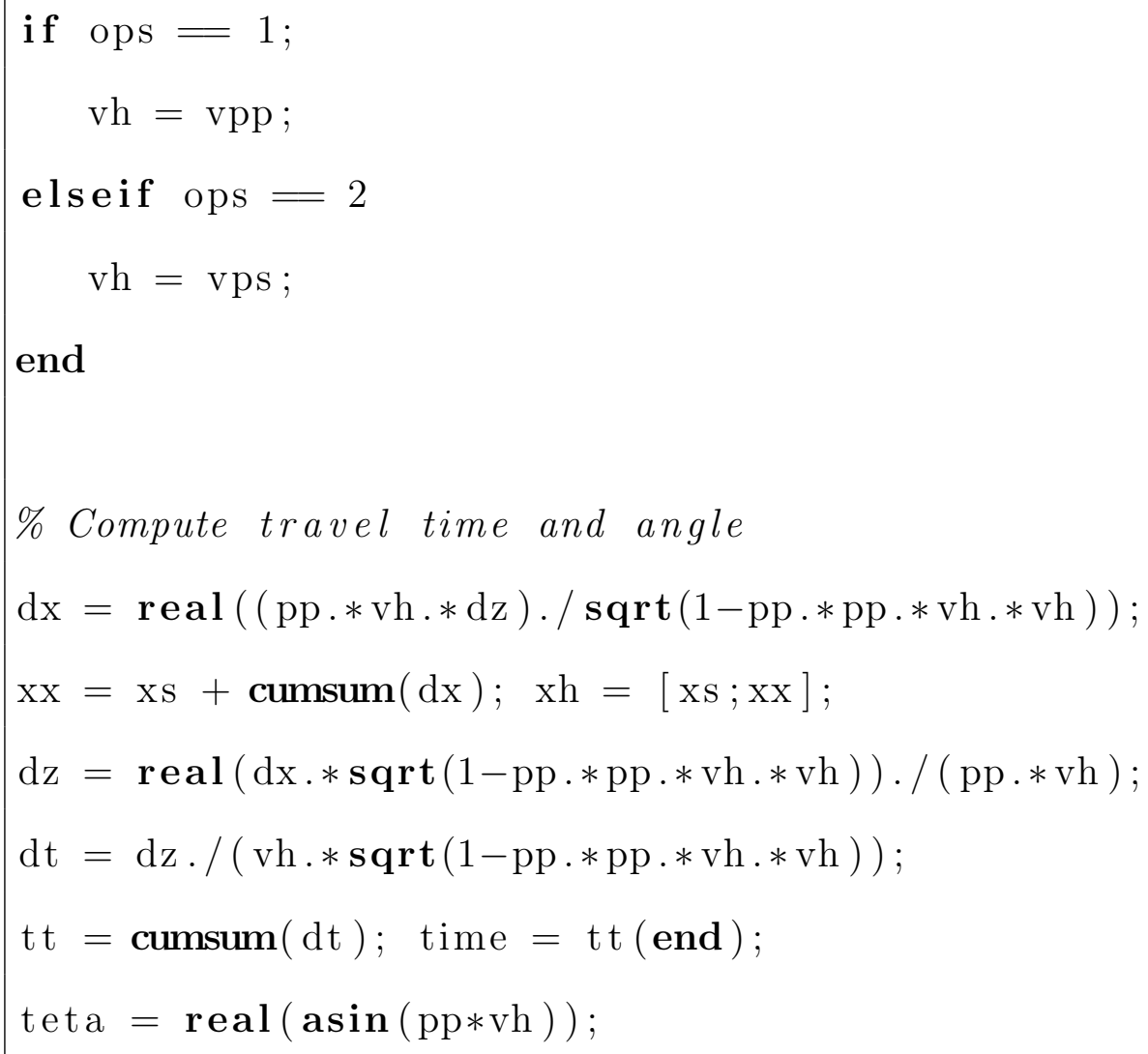




\section{$2.3 \quad$ S-wave velocity estimation}

In this section, I discuss how to invert S-wave velocity by using converted PS-wave traveltimes. The method includes four main steps: initial velocity model building, PS-wave ray tracing, PS-wave traveltime calculation, and S-wave velocity update.

\subsubsection{Initial S-wave velocity building}

The P-wave velocity can be accurately estimated first by using the picked traveltimes of direct P-wave from zero-offset VSP (Dillon and Thomson, 1984; Dillon et al., 1988; Hou et al., 2008). Then for estimating the S-wave velocity, the initial $V_{s}$ is calculated using Castagnas $V_{p}$ and $V_{s}$ relationship (Castagna et al., 1985). Different initial guesses of $V_{s}$ result in different inverted velocities. And the sensitivity test of using different initial models is presented in Chapter 3.1.

\subsubsection{PS-wave ray tracing}

The inversion method is based on ray tracing, thus we need to calculate converted wave traveltimes using ray paths (Geis et al., 1990). The shooting method is adopted for ray tracing. Figure 2.5 shows the diagram of ray paths computed using the shooting method. The red star is the source point and the blue square is the receiver point. The shooting method fixes one end of the ray path (source point), takes initial incidence angle and initial azimuth, and starts the initial ray tracing. The radius of target is analyzed using the bisection method, then the error is minimized and the 
ray path is linearly interpolated by the program. After setting a new ray parameter, the program shoots new rays and analyzes the radius of target again until the ray path achieves the end point (receiver point).

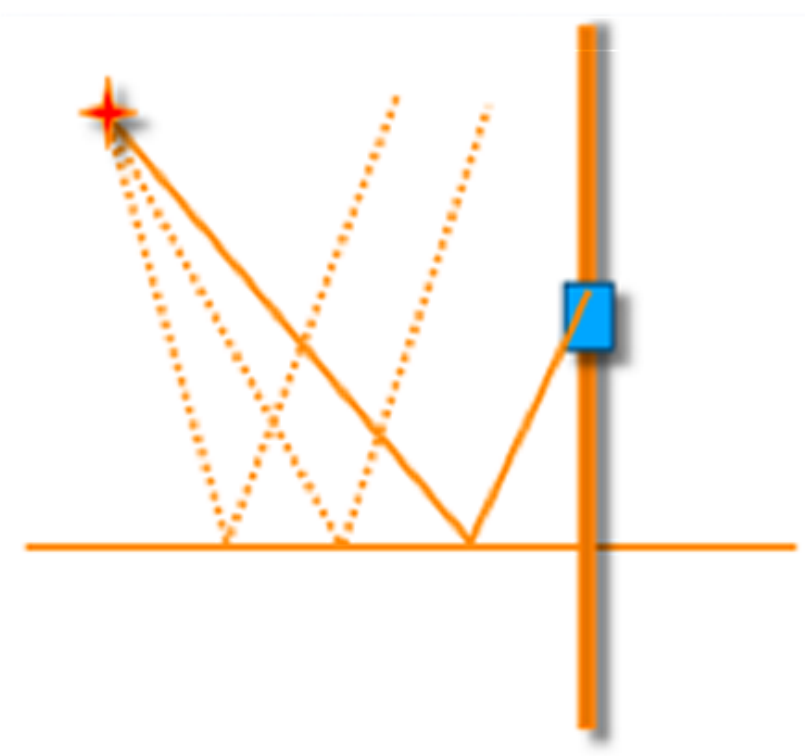

Figure 2.5: Diagram show of the shooting method.

\subsubsection{S-wave velocity inversion}

This section discusses how to invert S-wave velocity using PS-wave traveltimes. The inversion workflow is shown in Figure 2.6. And the inversion steps for two different cases are discussed as below. 


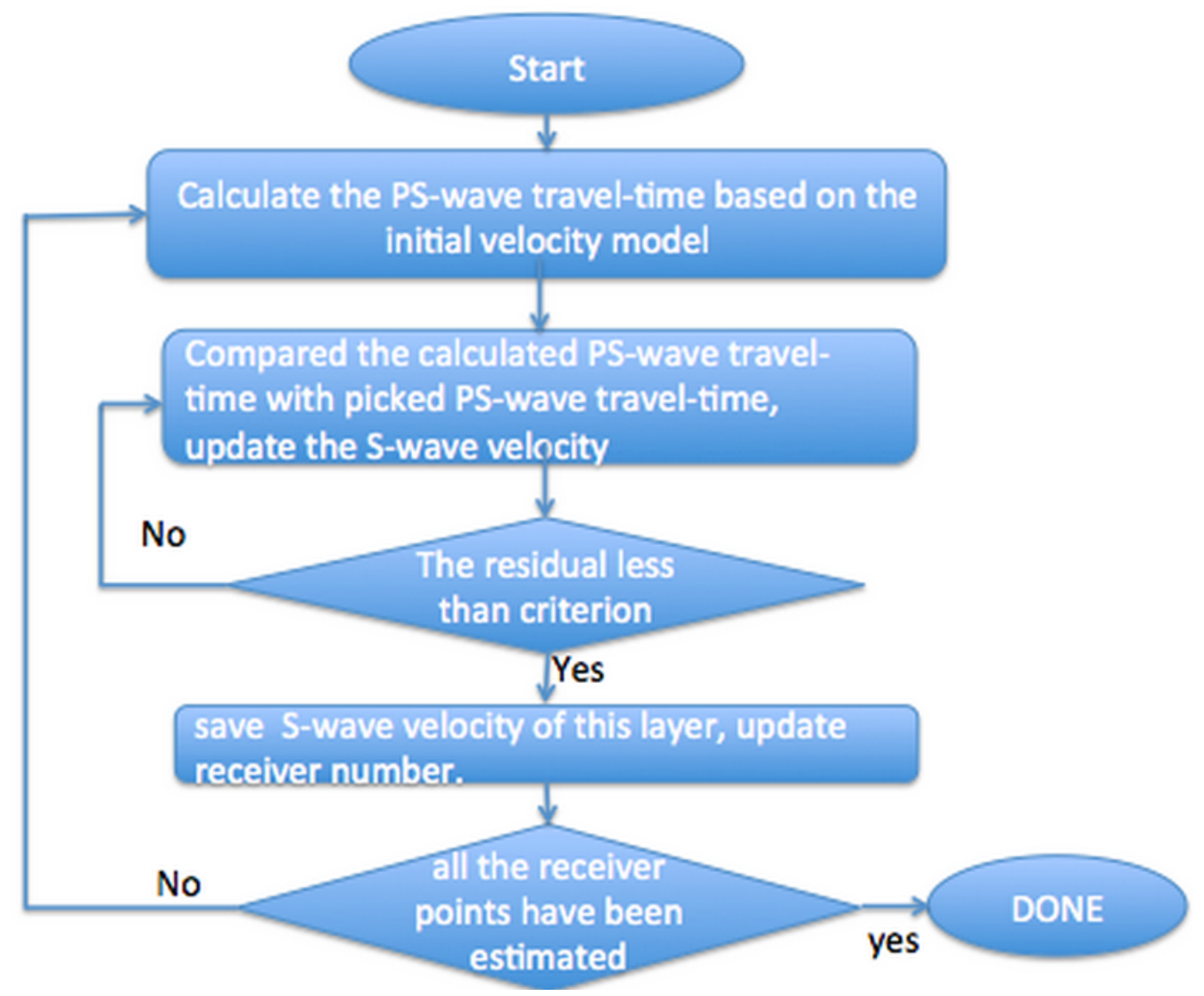

Figure 2.6: Workflow of converted wave traveltime inversion.

\subsubsection{Downgoing $\mathrm{P}$-wave to upgoing S-wave conversion}

1. Iteration starts from the $n t h$ receiver, and the S-wave velocity of the $n t h$ layer is inverted first. Based on the P-wave velocity, the initial S-wave velocity and the PS-wave ray path, we can calculate the PS-wave traveltime for the $n t h$ receiver.

2. Compare the calculated PS-wave traveltime with the picked one, and update the S-wave velocity using the traveltime difference. 
3. When the difference between the calculated and picked PS-wave traveltimes is less than a given criterion, stop the iteration and save the S-wave velocity of the $n$th layer.

4. Go back to step 1, calculate the PS-wave traveltime of the $(n-1)$ th receiver using the calculated S-wave velocity of the $n t h$ receiver.

5. When traveltimes for all receivers are calculated, we can obtain the whole Swave velocity all different receiver depths.

Note that here upgoing converted PS-wave is assumed, such that the inversion starts from the last receiver point and iteratively goes up to the first receiver point. That is, the S-wave velocity of a single layer is updated at each iteration.

\subsubsection{Downgoing $\mathrm{P}$-wave to downgoing $\mathrm{S}$-wave conversion}

The inversion steps by using downgoing converted S-wave are similar to that of using upgoing converted S-wave. The only difference is that the inversion starts from the first downgoing converted wave receiver to the last downgoing converted wave receiver. 


\section{Chapter 3}

\section{Synthetic data test}

In this chapter, two synthetic models are designed and tested to validate the S-wave velocity inversion method. The first model is used to test the sensitivities of different initial S-wave velocity models to inversion results. The second one is used to test the sensitivities of errors in picked traveltimes to the inversion.

\subsection{Synthetic model I}

In order to validate this method, a simple layer model is tested. Figure 3.1 shows the velocity model with a source at $500 \mathrm{~m}$ offset. The red star is the shot location and the green squares are VSP receivers. The total number of receivers is 18 with an interval of $80 \mathrm{~m}$. The velocity within each layer is constant. The converted PS-wave ray paths associated with this source-receivers geometry are overlaid on the model, where the black lines are downgoing $\mathrm{P}$-wave and the red lines are upgoing S-wave. 


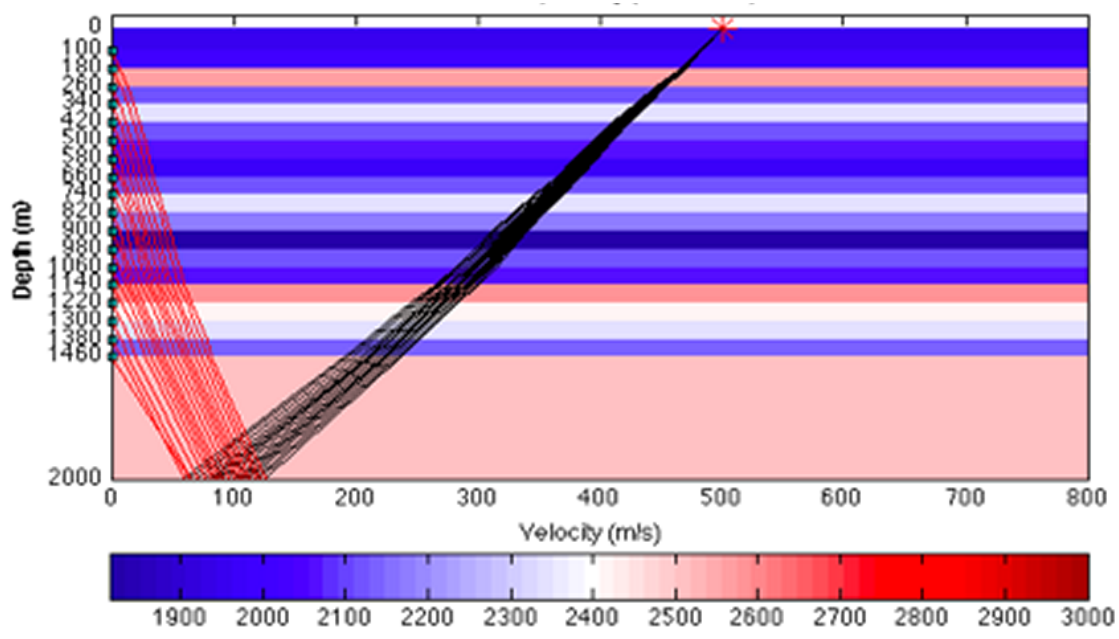

Figure 3.1: Synthetic model I and PS-wave ray paths of a VSP shot.

The PS-wave ray paths are obtained from PS-wave ray tracing using shooting method. Based on the ray paths, $V_{p}$ and $V_{s}$, the PS-wave traveltimes can be computed using equation 2.3. Table 3.1 displays the model information as well as the computed PS-wave traveltimes.

In order to know the sensitivities of different initial $V_{s}$ models to the inversion, I test the inversion with two different initial velocities. First, I set the initial $V_{s}=$ $0.5 * V_{p}$, and the inversion result is shown in Figure 3.2. The red curve is the initial guess of $V_{s}$; the blue curve is the true $V_{s}$; and the green curve is inverted $V_{s}$.

Figure 3.3 shows the differences between the true $V_{s}$ and the inverted $V_{s}$ using initial guess 1 . The mismatches at both shallow and deep depth are caused by that there are no receiver at depth of $0 m-100 m$ and $1460 m-2000 m$. And the RMS error between true $V_{s}$ and the inverted $V_{s}$ using initial guess 1 is $9.6 \mathrm{~m} / \mathrm{s}$.

Then I test a smaller initial $V_{s}=0.3 * V_{p}$, which is closer to the true $V_{s}$ compared 
Table 3.1: Properties of synthetic model I.

\begin{tabular}{|c|c|c|c|}
\hline Depth $(m)$ & $V_{p}(\mathrm{~m} / \mathrm{s})$ & $V_{s}(\mathrm{~m} / \mathrm{s})$ & $T_{p s}(s)$ \\
\hline 0 & 1945.82 & 505.0172414 & $\backslash$ \\
\hline 100 & 2010.60 & 560.8620690 & 3.472591553 \\
\hline 180 & 2563.46 & 1037.465517 & 3.330104505 \\
\hline 260 & 2113.02 & 649.1551724 & 3.253278082 \\
\hline 340 & 2335.26 & 840.7413793 & 3.130222770 \\
\hline 420 & 2111.12 & 647.5172414 & 3.035308853 \\
\hline 500 & 2061.28 & 604.5517241 & 2.911948580 \\
\hline 580 & 1965.48 & 521.9655172 & 2.779798111 \\
\hline 660 & 2121.43 & 656.4051724 & 2.626688136 \\
\hline 740 & 2340.73 & 845.4568966 & 2.505012665 \\
\hline 820 & 2169.84 & 698.137931 & 2.410652964 \\
\hline 900 & 1813.66 & 391.0862069 & 2.296284736 \\
\hline 980 & 2120.58 & 655.6724138 & 2.091852593 \\
\hline 1060 & 2055.29 & 599.387931 & 1.970055485 \\
\hline 1140 & 2586.86 & 1057.637931 & 1.836786040 \\
\hline 1220 & 2424.07 & 917.3017241 & 1.761507760 \\
\hline 1300 & 2335.94 & 841.3275862 & 1.674618234 \\
\hline 1380 & 2145.20 & 676.8965517 & 1.579834035 \\
\hline 1460 & 2508.62 & 990.1896552 & 1.461897212 \\
\hline 2000 & 2508.62 & 1413.793103 & \\
\hline & & & \\
\hline
\end{tabular}




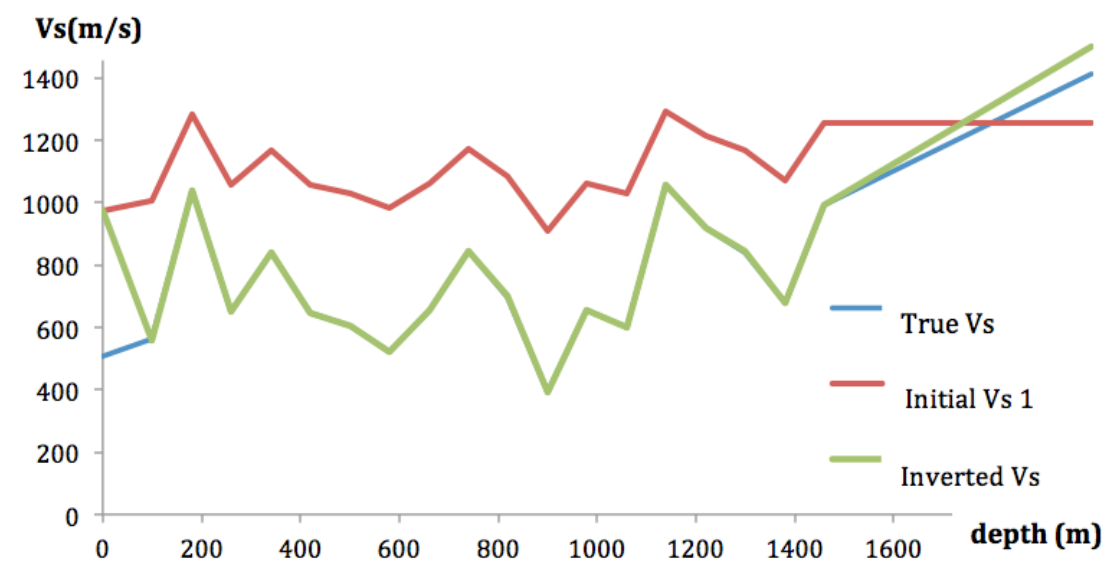

Figure 3.2: Inversion result using initial guess 1.

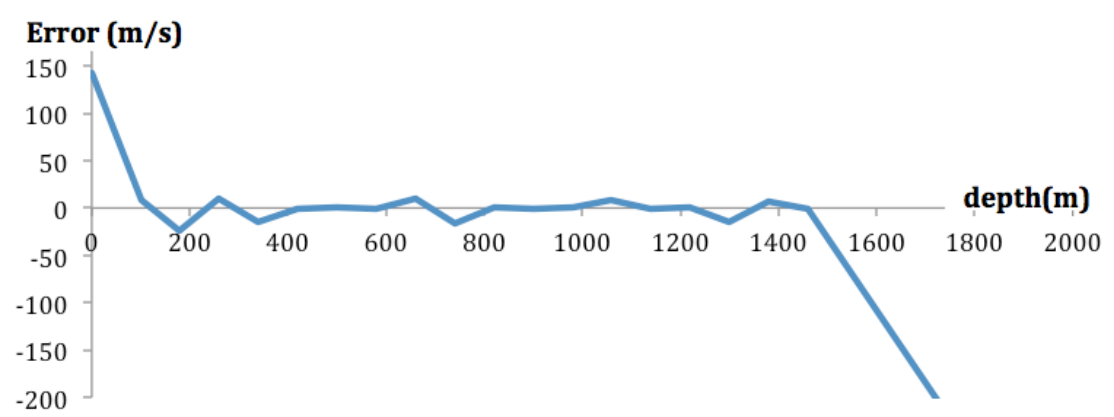

Figure 3.3: Differences between true $V_{s}$ and inverted $V_{s}$ using initial guess 1.

to the first initial guess. Figure 3.4 shows the corresponding inversion result. And the differences between true $V_{s}$ and the inverted $V_{s}$ using initial guess 2 are shown in Figure 3.5. The RMS error with initial guess 2 reduces to $0.72 \mathrm{~m} / \mathrm{s}$.

Comparing inversion results using the two different initial models, it is obvious that the closer of the initial $V_{s}$ to the true $V_{s}$, the less of the velocity residual. 


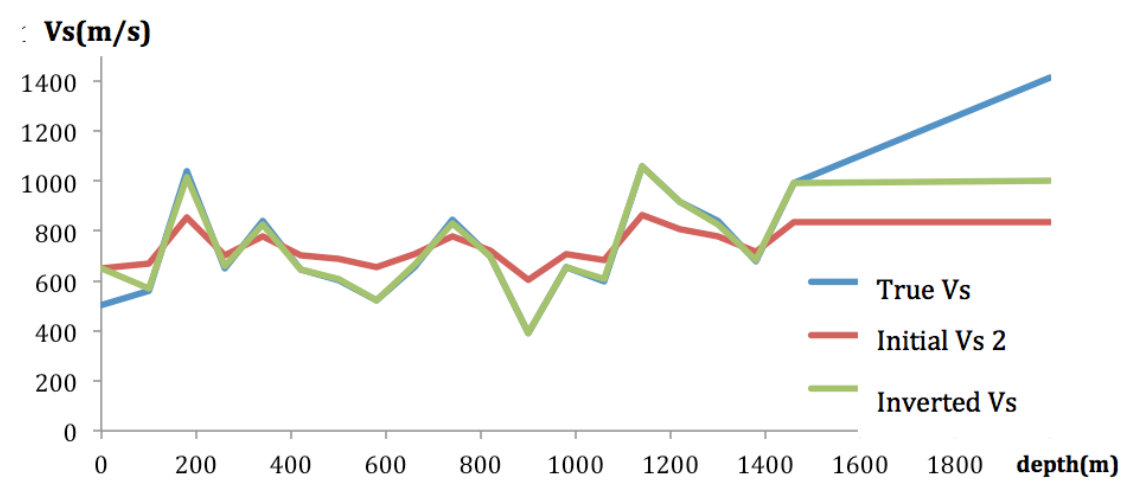

Figure 3.4: Inversion result using initial guess 2 .

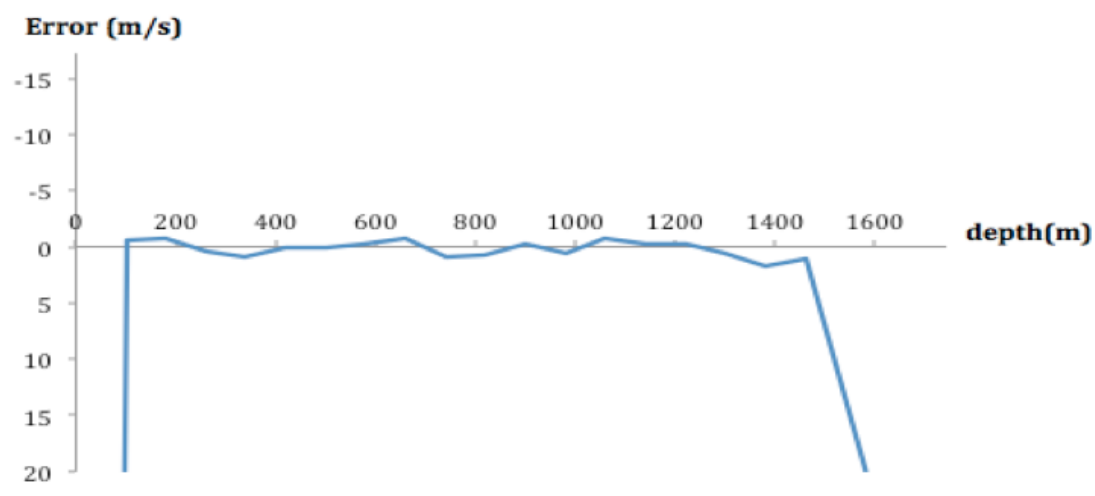

Figure 3.5: Differences between true $V_{s}$ and inverted $V_{s}$ using initial guess 2 .

\subsection{Synthetic model II}

When processing field data, we cannot always get the exact traveltimes due to the fair quality of seismic data. Besides, the picking errors vary from interpreter to interpreter. To simulate the real case, I use synthetic model II to test the sensitivities of traveltime picking errors to the inversion.

The same initial $V_{s}$ is used but with different traveltime pickings. First, I use the calculated traveltime (ground truth) for inversion; then I use traveltimes picked from the modeled PS-wave seismogram with random noise, where picking errors are 
assumed.

Figure 3.6 shows the synthetic model II with a source at $1000 \mathrm{~m}$ offset. The red star is the shot location, and the green squares are receiver locations. The number of receivers is 18 . And the receiver interval is $80 \mathrm{~m}$. The velocity within each layer is constant. The PS-wave ray paths are also displayed on the figure, where the black ray paths are corresponding to the downgoing $\mathrm{P}$-wave, and the red ray paths are associated with the upgoing S-wave. The PS-wave conversion points are at $1060 \mathrm{~m}$ depth.

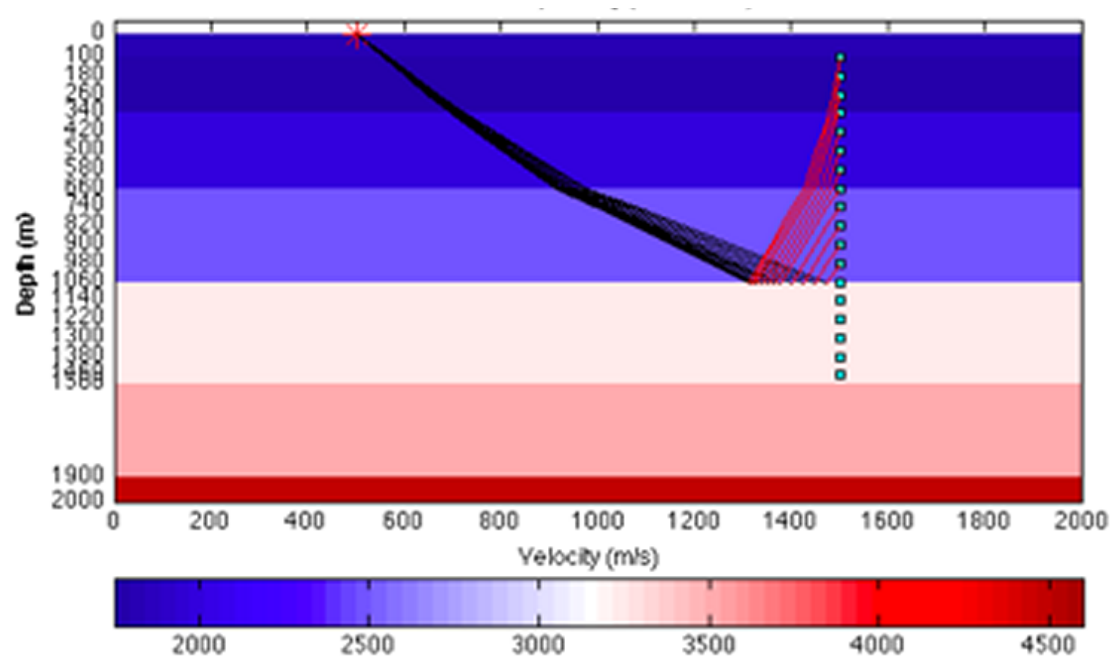

Figure 3.6: Synthetic model II and PS-wave ray paths of a VSP shot.

The true PS-wave traveltimes based on the ray paths are calculated and inverted first. To simulate the real case, I also manually pick the PS-wave traveltimes from the seismogram. Figure 3.7 shows the PS-wave seismogram with random noise associated with synthetic model II and the source-receivers geometry. The picked traveltimes are displayed as the red curve on the seismogram. Table 3.2 displays the differences 
between the calculated PS-wave traveltimes (ground truth) and the picked PS-wave traveltimes. And the picking errors are within $-10 \mathrm{~ms}$ to $13 \mathrm{~ms}$.

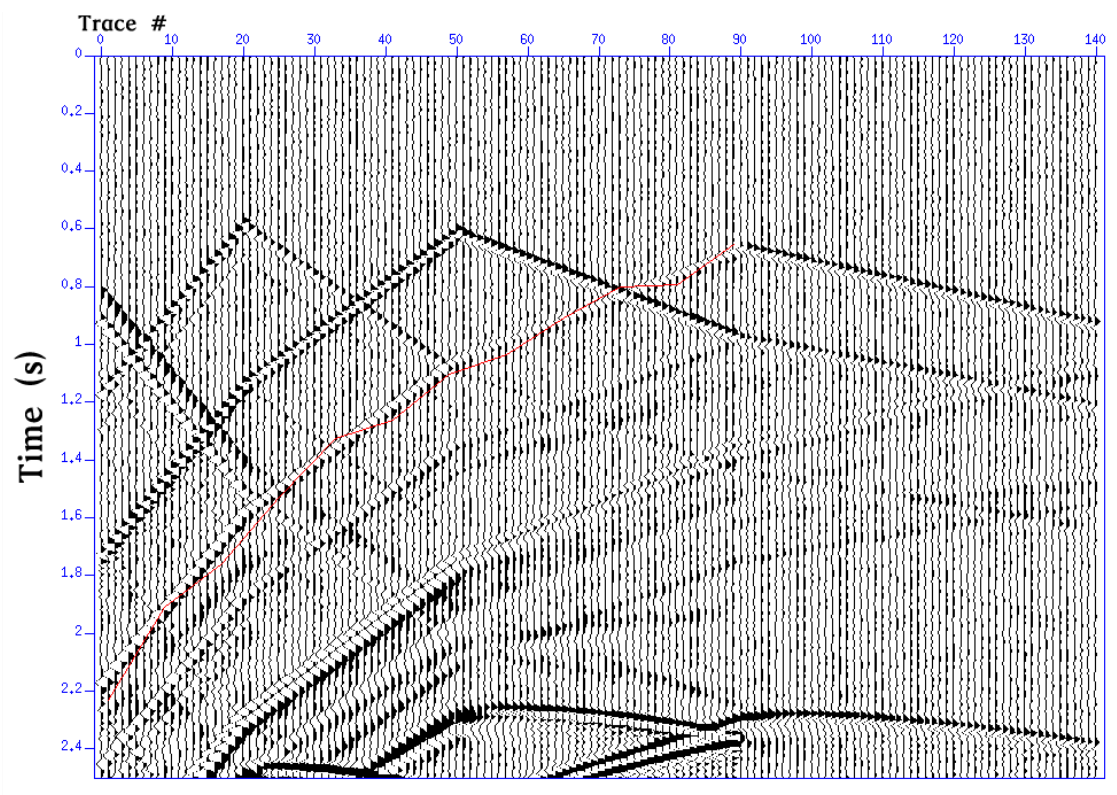

Figure 3.7: PS-wave seismogram obtained from elastic finite-difference modeling with random noise.

Figure 3.8 shows the inversion result using the exact traveltimes. The blue curve is the true S-wave velocity, and the red curve is the inverted one. The inverted Swave velocity is very close to the true one, except the shallow part $(0 m-100 m)$ where there is no receiver. Figure 3.9 displays the inversion result using the picked traveltimes with errors. The RMS error between the picked traveltimes and true traveltimes is $7.019 \mathrm{~ms}$. And the RMS error between true $V_{s}$ with the inverted $V_{s}$ is $92.3 \mathrm{~m} / \mathrm{s}$. 
Table 3.2: Traveltimes associated with synthetic model II.

\begin{tabular}{|c|c|c|}
\hline True $T_{p s}(m s)$ & Picked $T_{p s}(m s)$ & Difference $(m s)$ \\
\hline 2369.385028 & 2367.950087 & -1.43494014 \\
\hline 2132.513529 & 2129.391069 & -3.122459918 \\
\hline 1895.652509 & 1887.537096 & -8.115413222 \\
\hline 1658.802017 & 1666.175004 & 7.372986605 \\
\hline 1515.634603 & 1528.342035 & 12.70743197 \\
\hline 1372.496332 & 1371.384175 & -1.112156869 \\
\hline 1229.387392 & 1235.082178 & 5.694785895 \\
\hline 1086.307945 & 1077.717945 & -8.589999668 \\
\hline 1008.454126 & 1016.639760 & 8.185634054 \\
\hline 930.7011399 & 931.9703547 & 1.269214736 \\
\hline 853.0496010 & 858.4705334 & 5.420932412 \\
\hline 775.4998074 & 765.9462444 & -9.55356293 \\
\hline
\end{tabular}




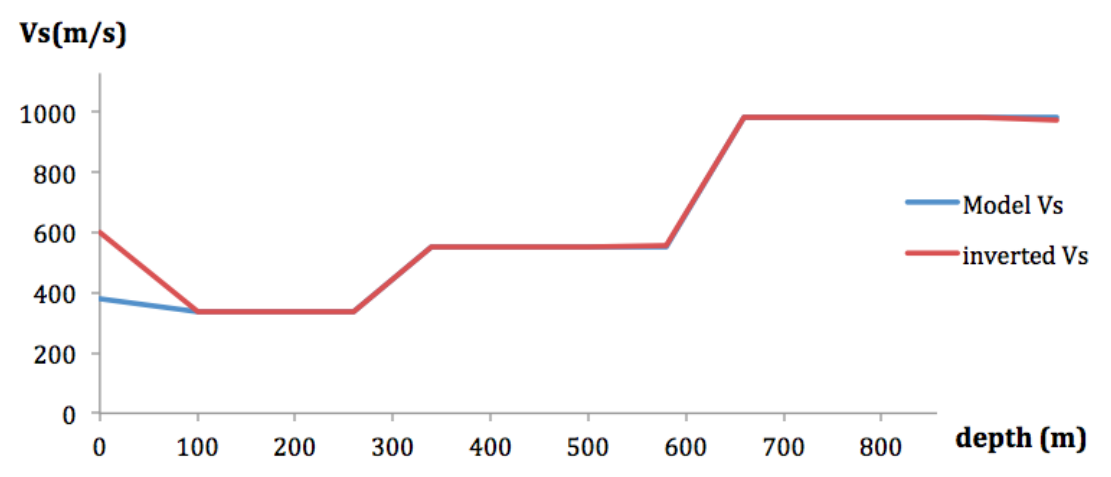

Figure 3.8: Inverted result using calculated PS-wave traveltimes.

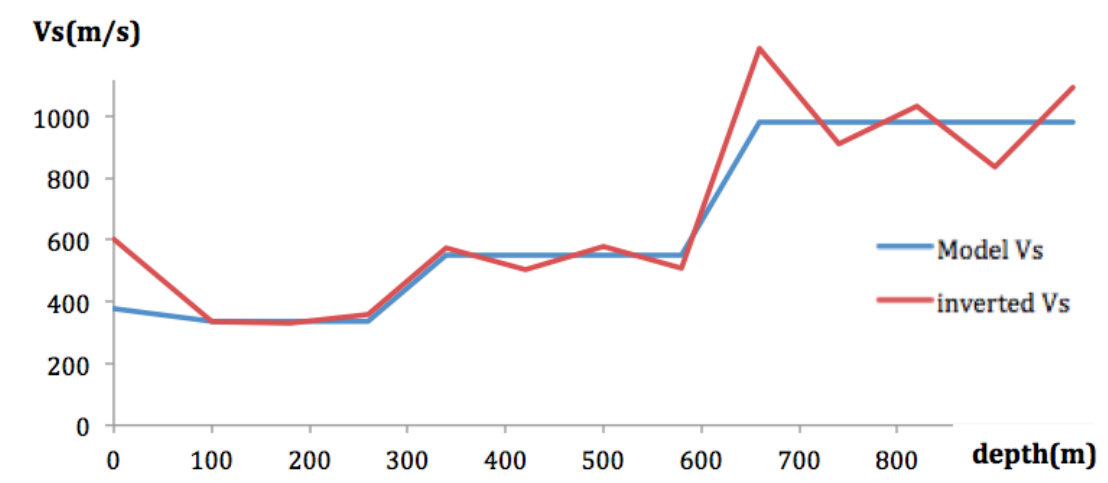

Figure 3.9: Inverted result using picked PS-wave traveltimes with errors.

\subsection{Summary}

According to the inversion results discussed above, the following conclusions can be drawn. First, equation 2.3 is valid for calculating upgoing PS-wave traveltime. Second, when the first break times of direct S-wave are not easy to pick, the proposed method can be a good alternative for building an S-wave velocity model. Third, different initial models result in different inverted velocities; and the better of the initial guess, the closer of the inverted one to the true one. In addition, accuracy of traveltime pickings is critical for achieving a precise estimate of S-wave velocity. 


\section{Chapter 4}

\section{Field data test}

In this chapter, I start with the introduction of the 3C VSP field dataset. After that, the VSP data processing workflow is discussed. The workflow includes the processing of P-wave, converted wave, zero-offset, and offset VSPs. Finally, the S-wave velocity is inverted using converted waves in the VSP data with method discussed in Chapter 2.

\subsection{Introduction}

The 3C VSP field dataset is provided by Borehole Seismic Center of BGP, China. The studying area is located in the Huabei oil field, China. Figure 4.1 shows the geographical location of this oil field. Figure 4.2a shows the time migrated surface seismic section of this area, and Figure 4.2 shows the corresponding geological interpretation. 


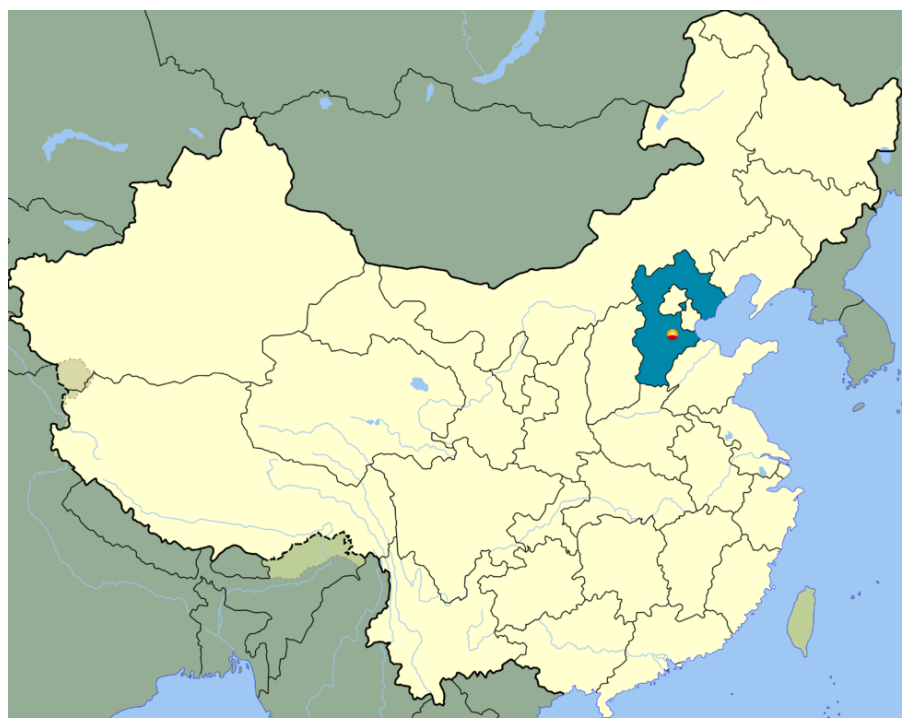

Figure 4.1: Geographic location of the Huabei oil field.

The VSP data was acquired in 2008. The maximum depth of the vertical well is about $2080 \mathrm{~m}$. There is no casing cover from depth $910 \mathrm{~m}$ to $2080 \mathrm{~m}$. The raw offset VSP has strong converted waves, which are generated from the reflector at $910 \mathrm{~m}$ depth. The lithology at this depth changes from sandstone/mudstone to dolomitic limestone. Figure 4.3 shows the vertical components of zero-offset VSP and offset VSP. Figure 4.4 displays well log information about this well, with density showing in purple, gamma ray (GR) in blue, and acoustic (AC) in red. Lithology information about this well is displayed on the right of Figure 4.4. And generalized stratigraphy of this area is given in Table 4.1.

The main hydrocarbon bearing sands are at around $2000 \mathrm{~m}$ depth, and the hydrocarbon entrapment is mainly strati-structural. The traps are formed by shale out zones of sands, associated with structural play that are considered to be the best locales for hydrocarbon entrapment in this area. 


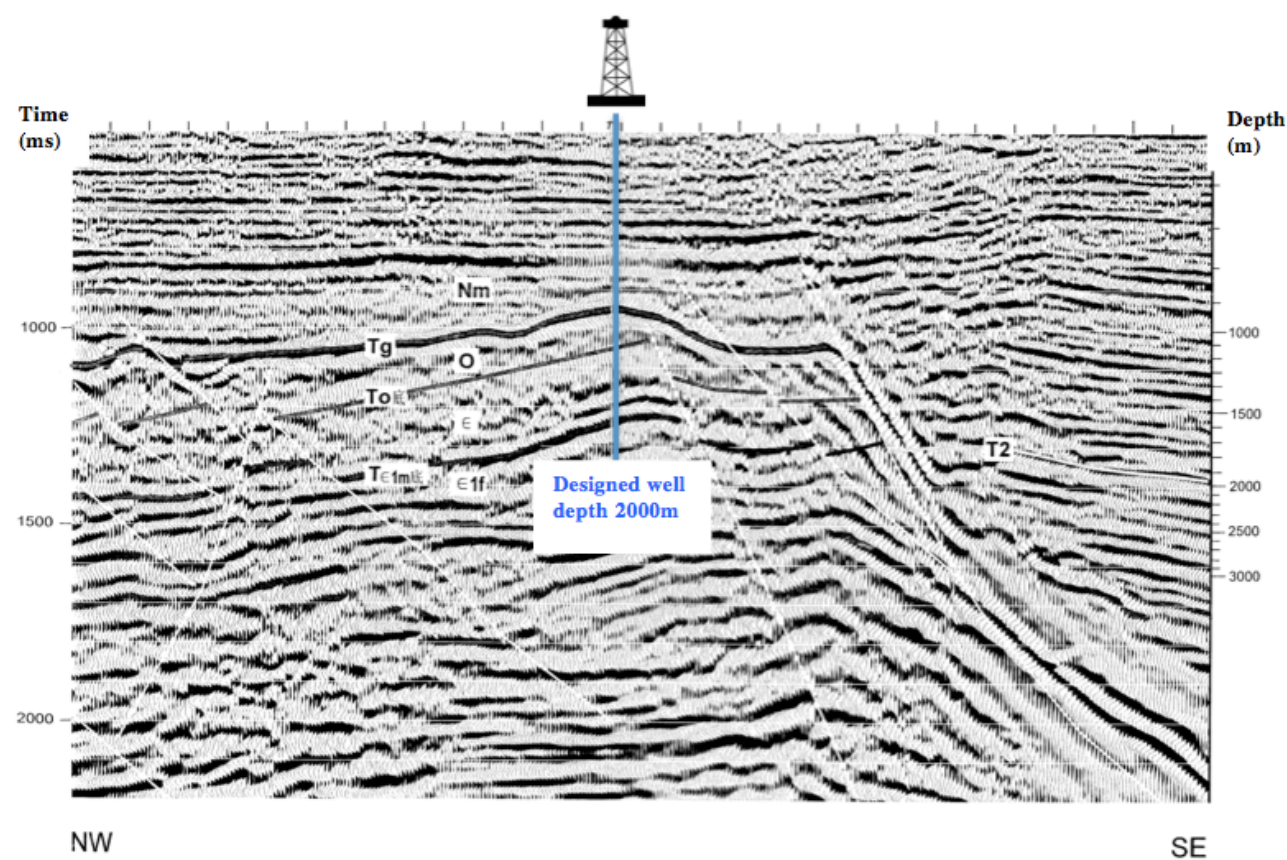

(a)

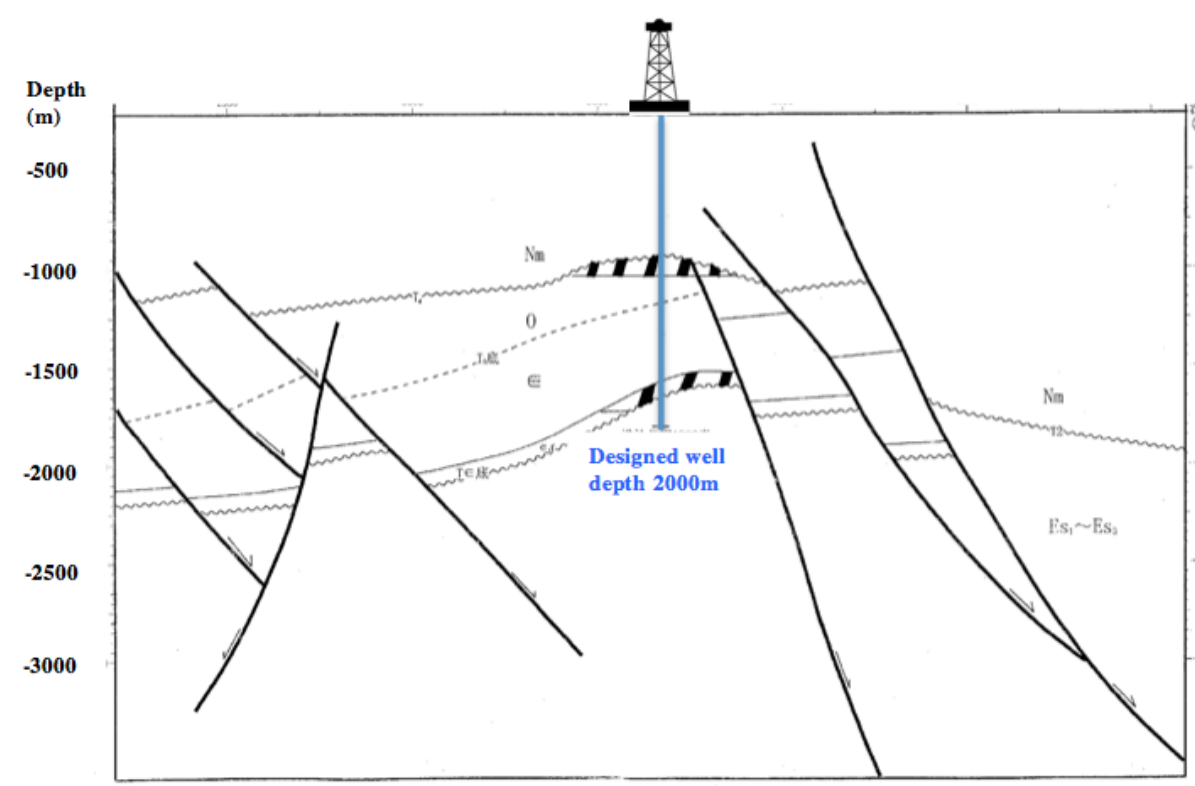

(b)

Figure 4.2: (a) A surface seismic section of the studying area. (b) Geological interpretation of (a). 


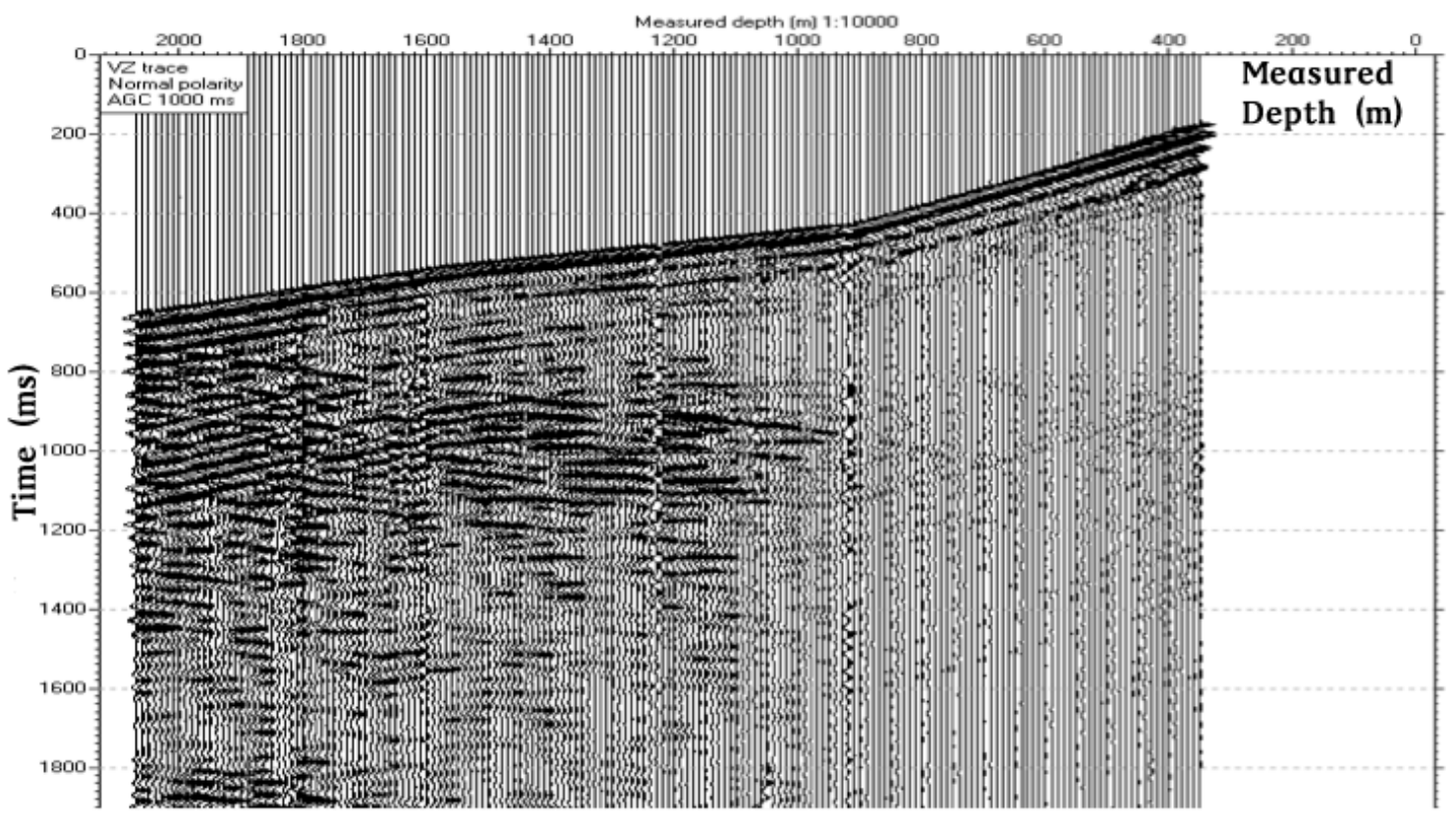

(a)

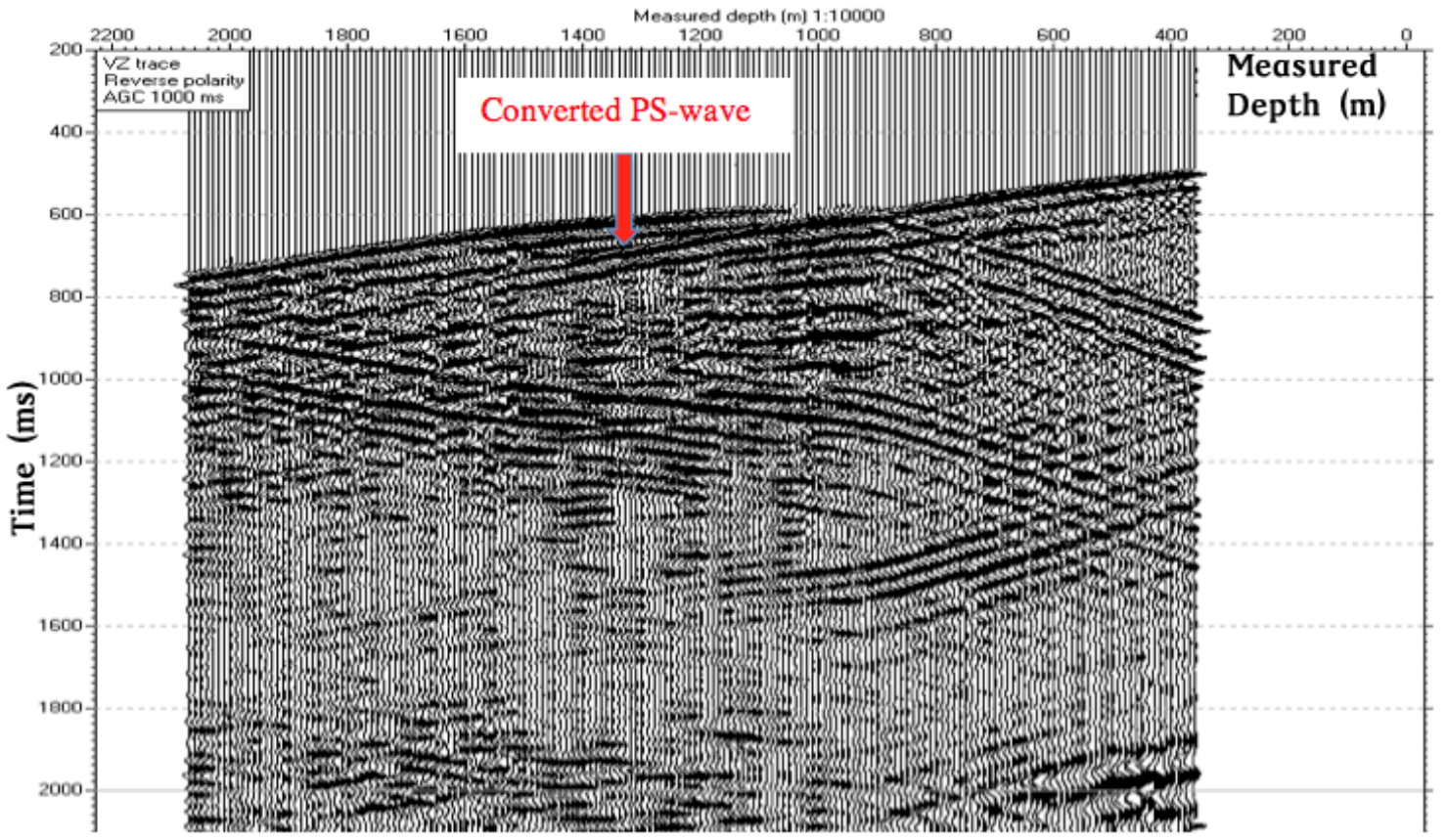

(b)

Figure 4.3: Vertical component of the (a) zero-offset VSP and the (b) offset VSP. 


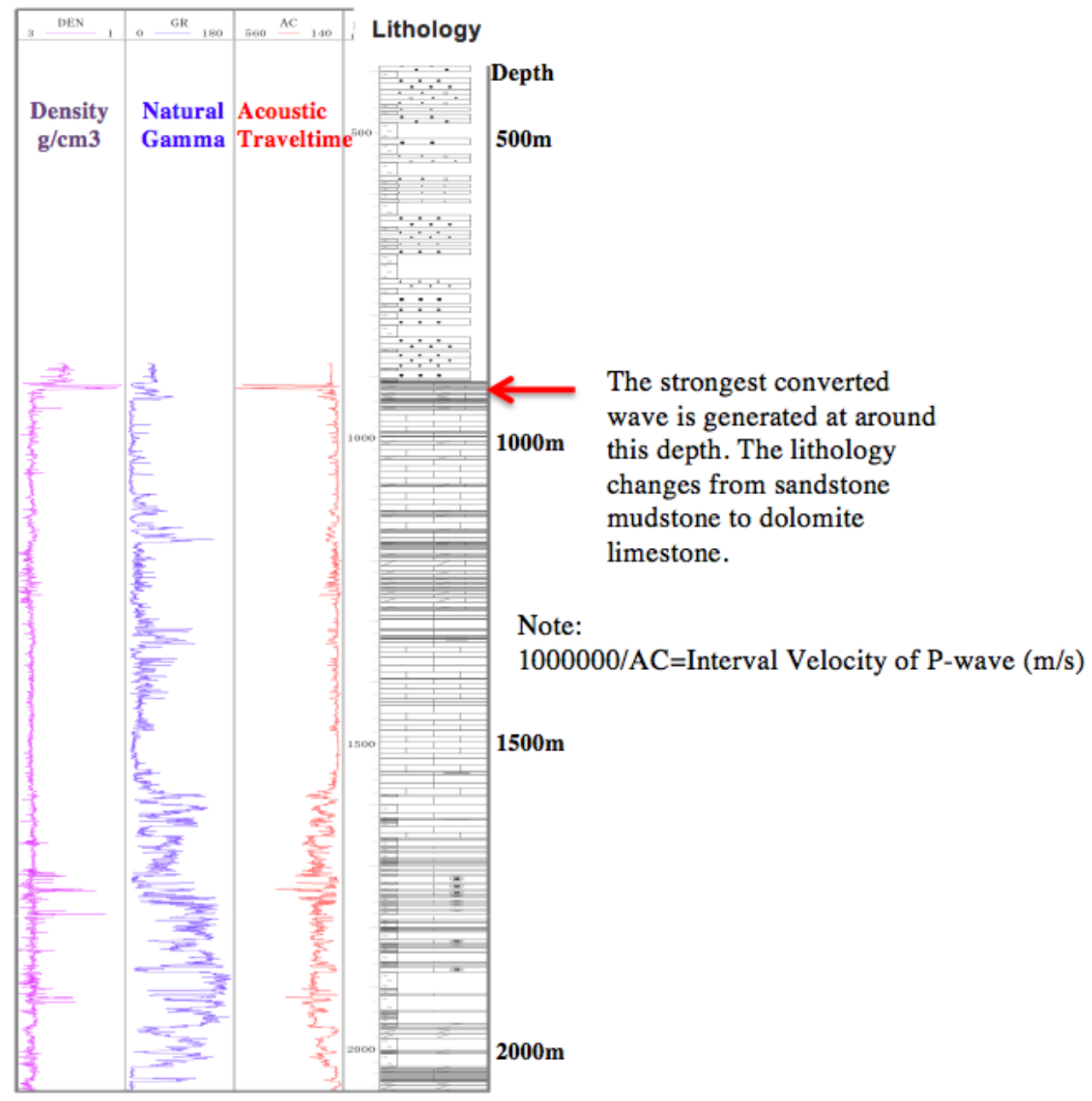

Figure 4.4: Density, GR and AC logs of this well.

Table 4.1: Generalized stratigraphy of the studying area.

\begin{tabular}{|c|l|l|l|l|}
\hline Era & Penod & Depth & Interval velocity & Lithology \\
\hline Cenozoic & Quaternary & $\begin{array}{l}\text { Surface to } \\
902 \mathrm{~m}\end{array}$ & $\begin{array}{l}\text { Around } \\
2180 \mathrm{~m} / \mathrm{s}\end{array}$ & $\begin{array}{l}\text { Sandstone } \\
\text { Mudstone }\end{array}$ \\
\hline \multirow{3}{*}{ Paleozoic } & Ordovician & $\begin{array}{l}920 \mathrm{~m} \text { to } \\
1583 \mathrm{~m}\end{array}$ & $\begin{array}{l}\text { Around } \\
6000 \mathrm{~m} / \mathrm{s}\end{array}$ & $\begin{array}{l}\text { Dolomite } \\
\text { limestone }\end{array}$ \\
\cline { 2 - 5 } & Cambrian & $\begin{array}{l}1570 \mathrm{~m} \text { to } \\
2080 \mathrm{~m}\end{array}$ & $\begin{array}{l}\text { Around } \\
4000 \mathrm{~m} / \mathrm{s}\end{array}$ & $\begin{array}{l}\text { Dolomite } \\
\text { limestone }\end{array}$ \\
\hline
\end{tabular}




\subsection{VSP acquisition parameters}

The 3C VSP survey includes one zero-offset VSP and one offset VSPs. The data acquisition parameters are listed in Table 4.2. And the geometry of this VSP survey is shown in Figure 4.5, where red points are source points and green line is the receiver line.

Table 4.2: Acquisition parameters of 3C zero-offset and offset VSPs.

\begin{tabular}{|l|l|}
\hline Source & Dynamite \\
\hline Offset distance of zero-offset VSP & $89 \mathrm{~m}$ \\
\hline Offset distance of offset VSP & $869 \mathrm{~m}$ \\
\hline Receiver depth & $350 \mathrm{~m}$ to $2070 \mathrm{~m}$ \\
\hline Receiver spacing & $10 \mathrm{~m}$ \\
\hline
\end{tabular}

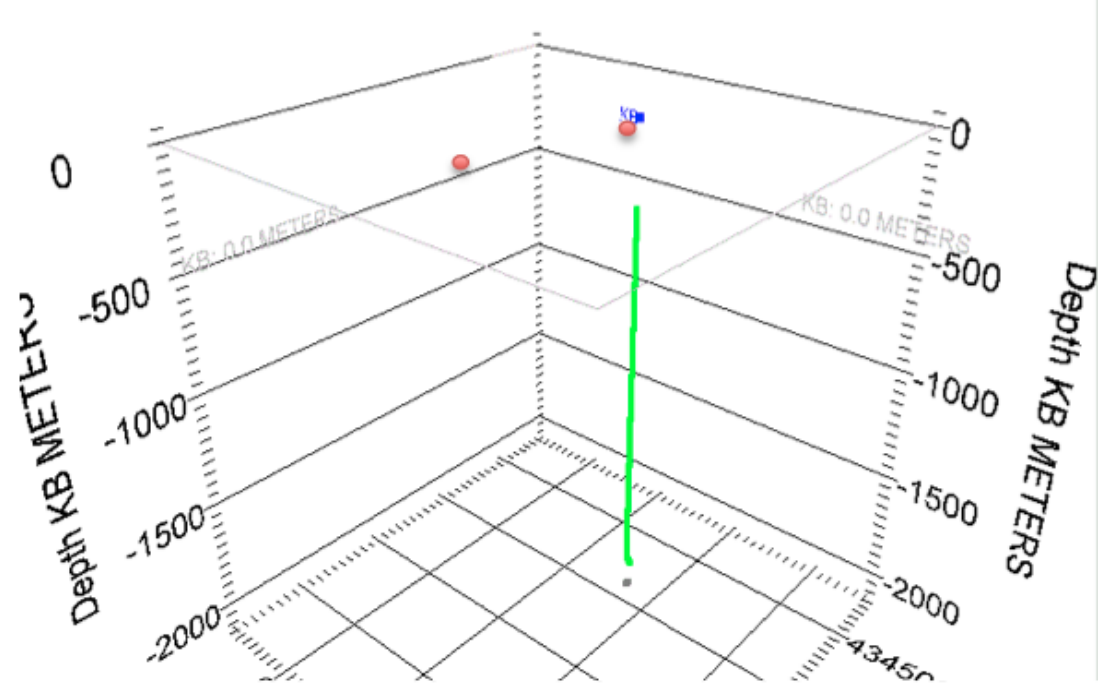

Figure 4.5: Shots and receivers distribution of the VSP survey. 


\subsection{Raw data analysis}

Figure 4.6a shows all three components of the raw zero-offset VSP data. The left panel is the vertical component, and middle and right panels are two horizontal components. The frequency spectrum of is showing on the bottom. On the $\mathrm{Z}$ component, the downgoing direct P-waves are clear to see, so that we can easily pick first break times of direct $\mathrm{P}$-wave to estimate the $\mathrm{P}$-wave velocity. Figure $4.6 \mathrm{~b}$ displays the same zero-offset VSP data after rotation. On the horizontal component (middle panel), the direct S-waves are still too weak to see, thus it is difficult to estimate the S-wave velocity by picking first break times of direct S-wave.

Figure $4.7 \mathrm{a}$ is similar as Figure 4.6 a except that the data is the raw offset VSP data. On the vertical component, both the downgoing $\mathrm{P}$-wave and upgoing $\mathrm{P}$-wave are obvious to see. Figure $4.7 \mathrm{~b}$ shows the offset VSP data after rotation. On the horizontal component (middle panel), the strong converted PS-waves are generated from seismic boundary at depth $910 \mathrm{~m}$ and can be easily identified.

\subsection{C VSP data processing}

In this section, I discuss the processing steps of the $3 \mathrm{C}$ VSP field data. The processing includes both P-wave and converted wave of zero-offset and offset VSPs. And data processing software such as ProMAX and VISTA are used. 


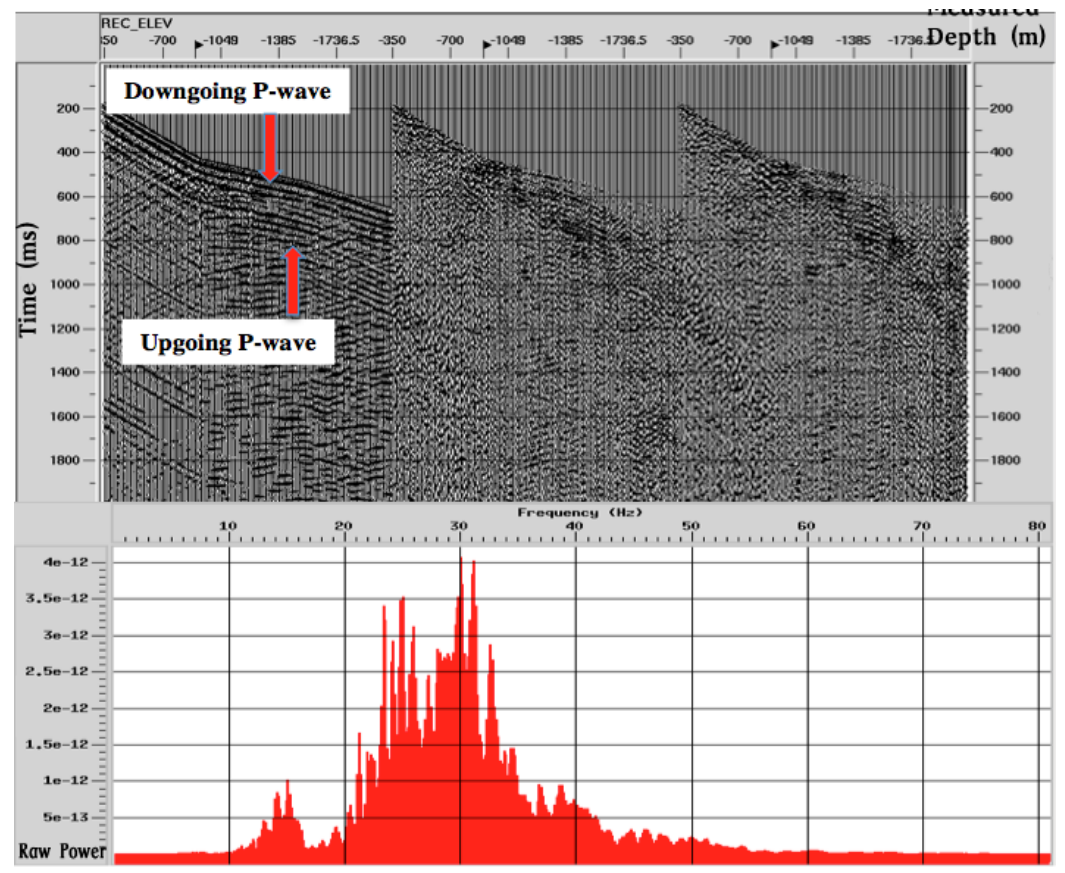

(a)

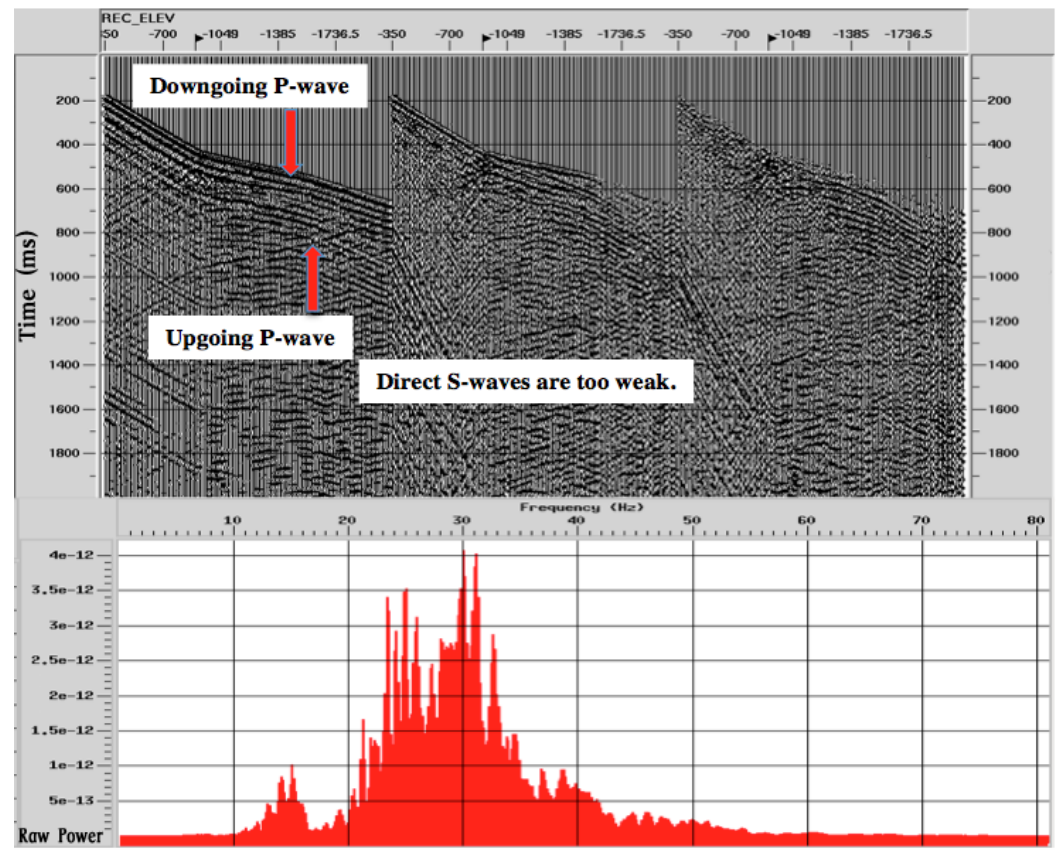

(b)

Figure 4.6: Raw zero-offset VSP data (a) before and (b) after rotation. 


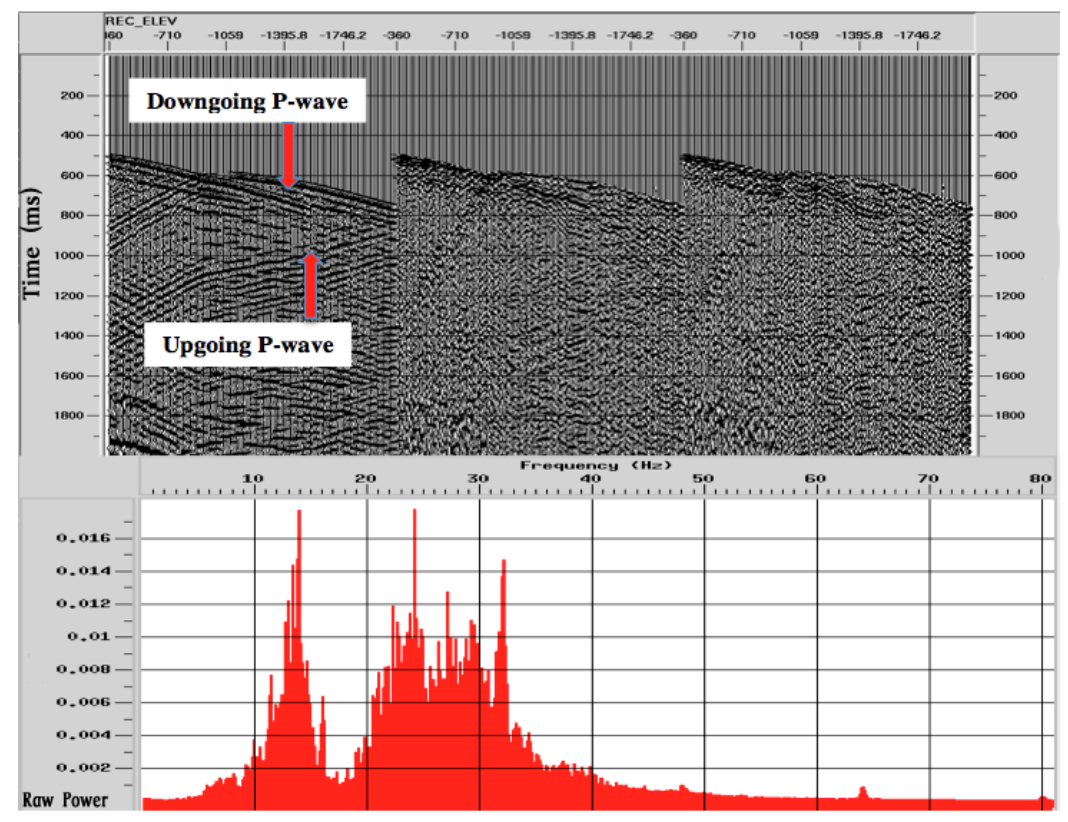

(a)

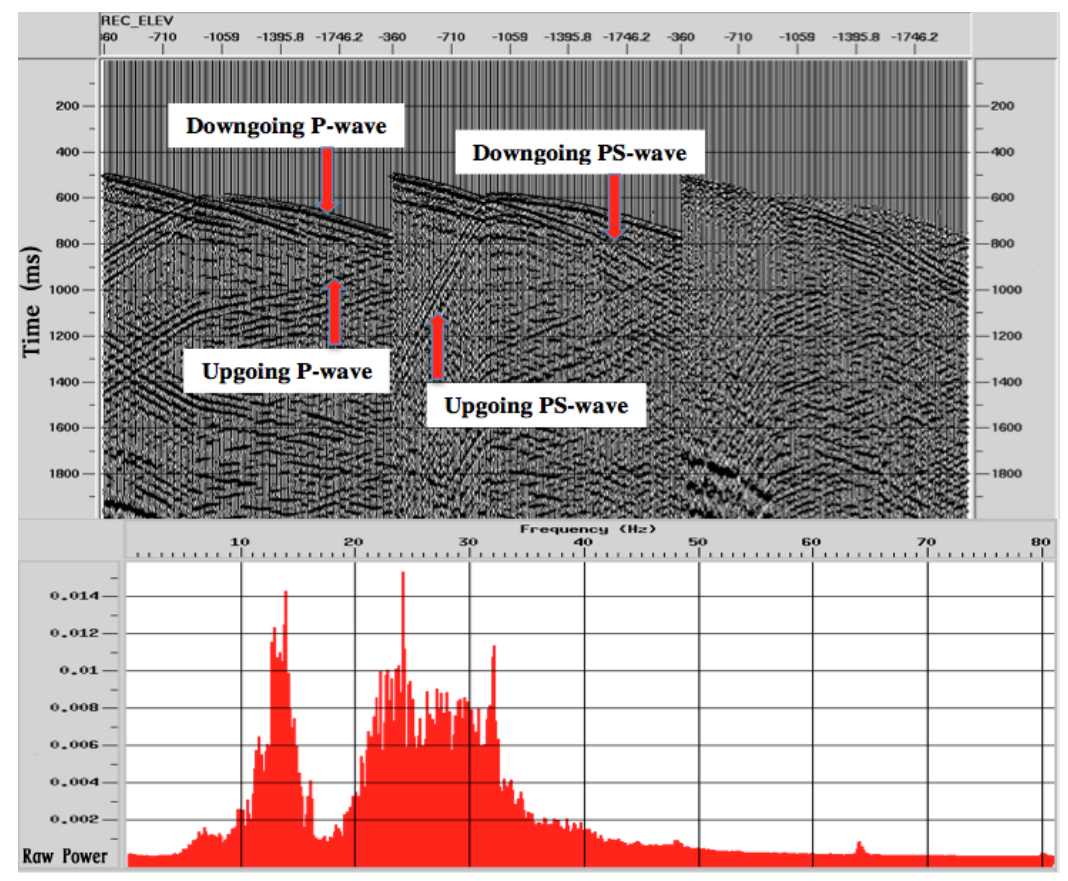

(b)

Figure 4.7: Raw offset VSP data (a) before and (b) after rotation. 


\subsubsection{Zero-offset VSP}

There are eight major steps for processing the zero-offset VSP data. They are explained in detail as below.

\section{Define VSP geometry}

Two geophones are used for acquiring the VSP data in this case. Channels 11 and 14 are two vertical components. Channels 9, 10, 12 and 13 are four horizontal components. For zero-offset VSP, the actual offset is $89 \mathrm{~m}$. The receiver depth is from $360 \mathrm{~m}$ to $2000 \mathrm{~m}$. The geometry of the zero-offset VSP survey is displayed in Figure 4.5. After properly building the geometry, we can display the 3C VSP raw data in ProMAX (see Figure 4.8).

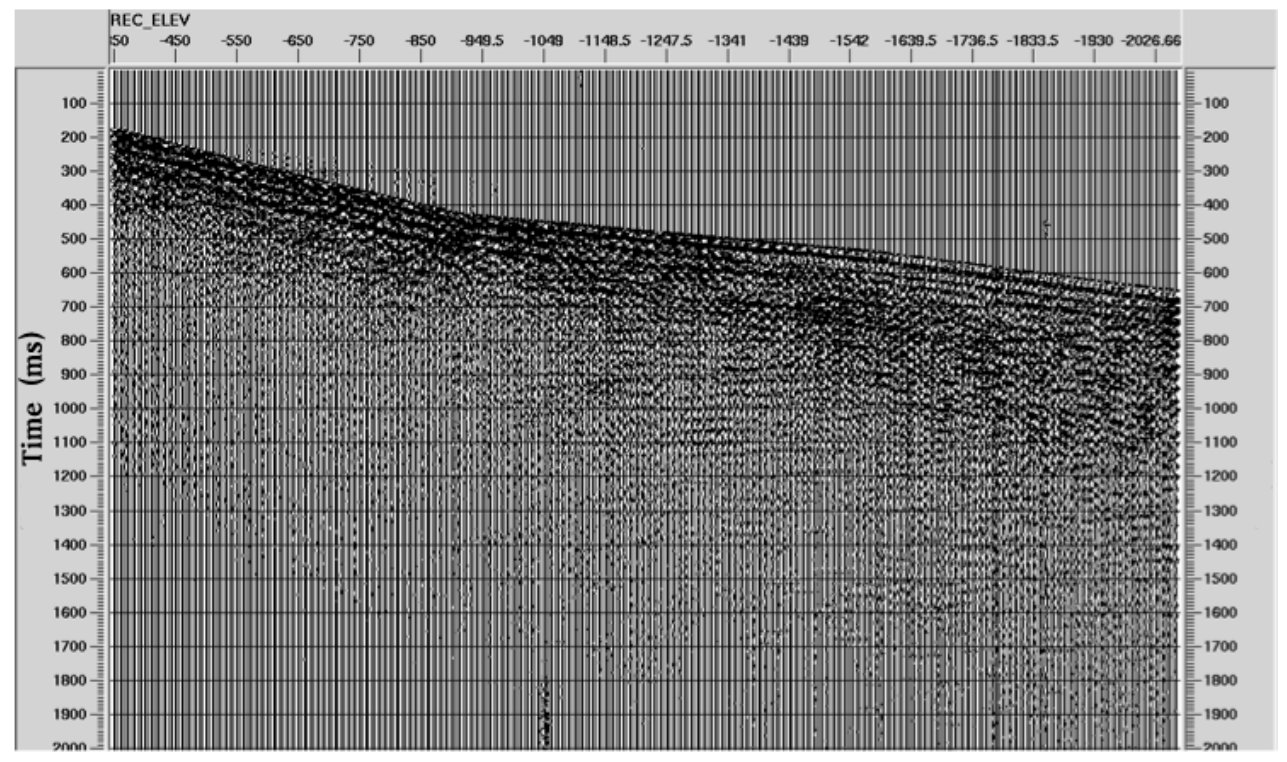

Figure 4.8: 3C zero-offset VSP raw data.

2. Keep vertical component trace 
Although VSP data are recorded using 3C geophones, the vertical component is the major channel used for data processing. In this step, I sort the input data and only keep the vertical component. Figure 4.9 shows the separated three components from the raw data. For the vertical component, the downgoing direct $\mathrm{P}$-wave and upgoing reflections are strong. We can easily pick the first break times of direct $\mathrm{P}$-wave to estimate the $\mathrm{P}$-wave velocity.

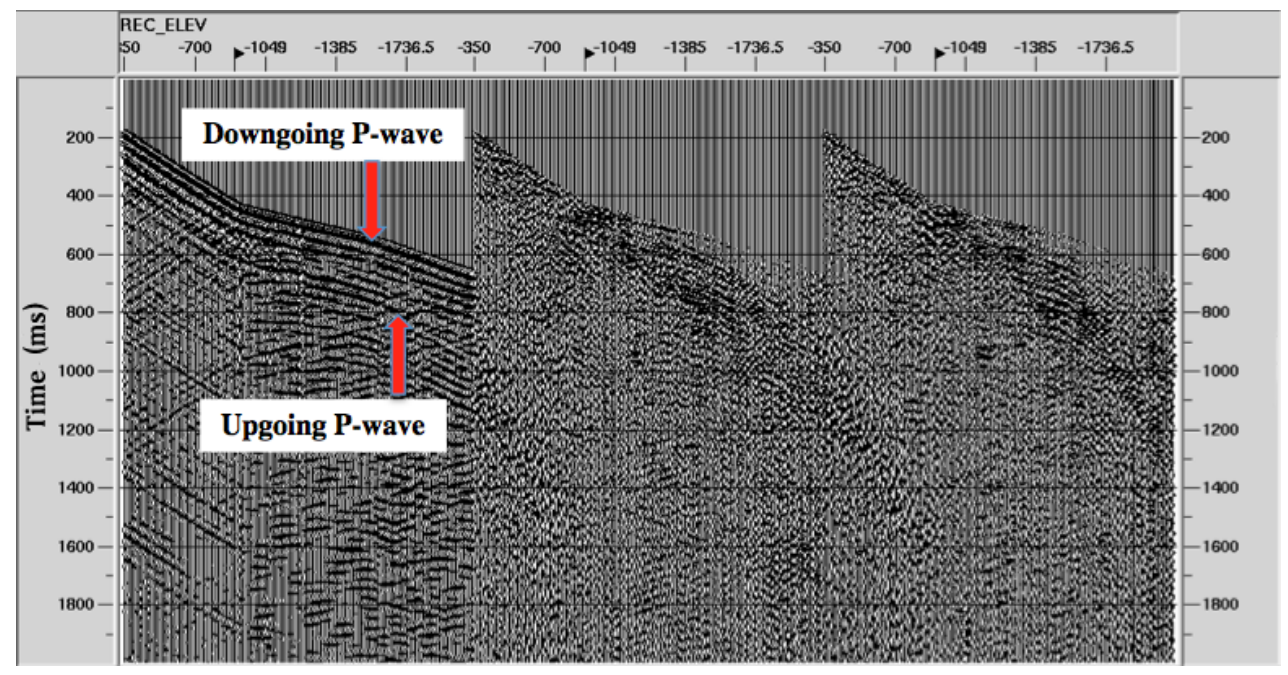

Figure 4.9: Separated vertical (left) and two horizontal (middle and right) components of the zero-offset VSP.

3. Pick first break times

First break times picking is an integral part of the VSP data processing. By using the picked traveltimes of the $\mathrm{P}$-wave, we can estimate the $\mathrm{P}$-wave velocity, interval velocity, and time-depth relationship. But the direct S-wave in the zero-offset VSP is too weak because of the dynamite source. It cannot be used to estimate the S-wave velocity. Figure 4.10 displays the picked first break times of direct P-wave. The blue curve shown in Figure 4.11 is the estimated 
P-wave interval velocity. The lithology changes from sandstone and mudstone to dolomite and limestone at around $910 \mathrm{~m}$, which explains the velocity jump at this depth.

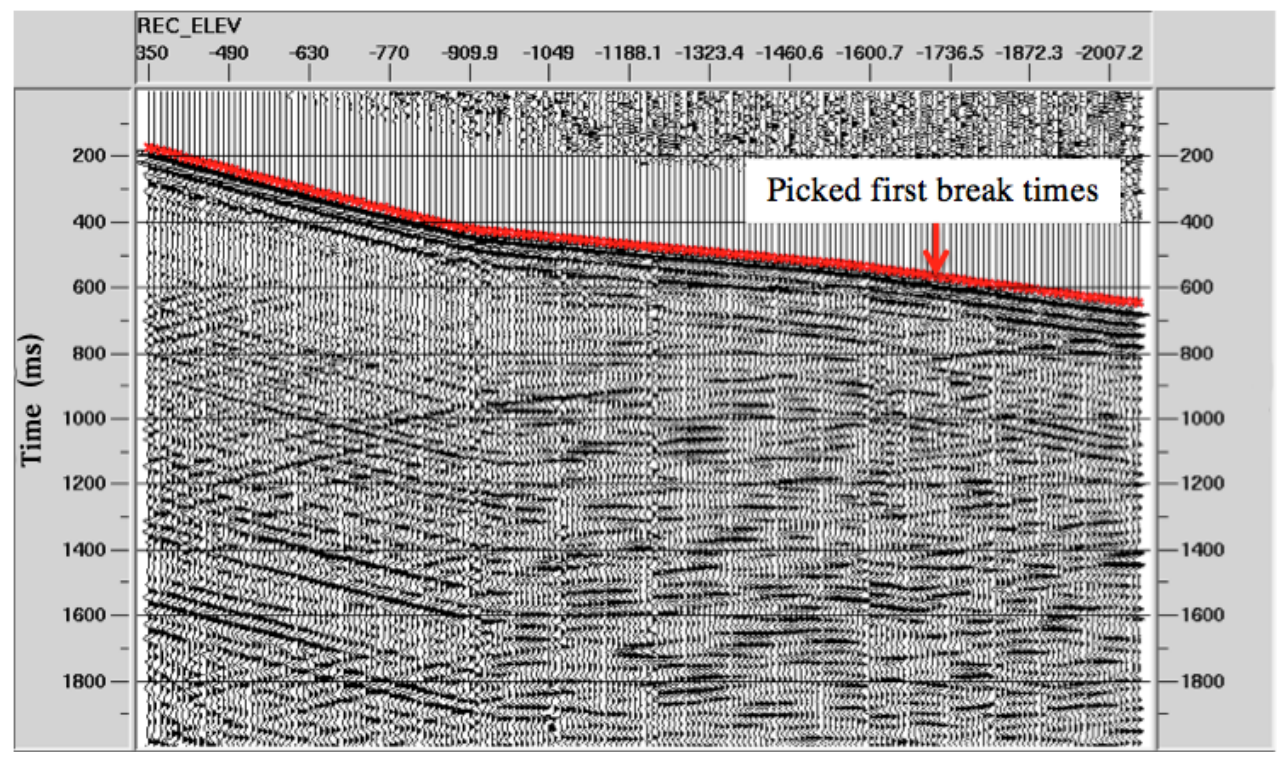

Figure 4.10: Vertical component of the zero-offset VSP data. The red line is the picked first break times of direct P-wave.

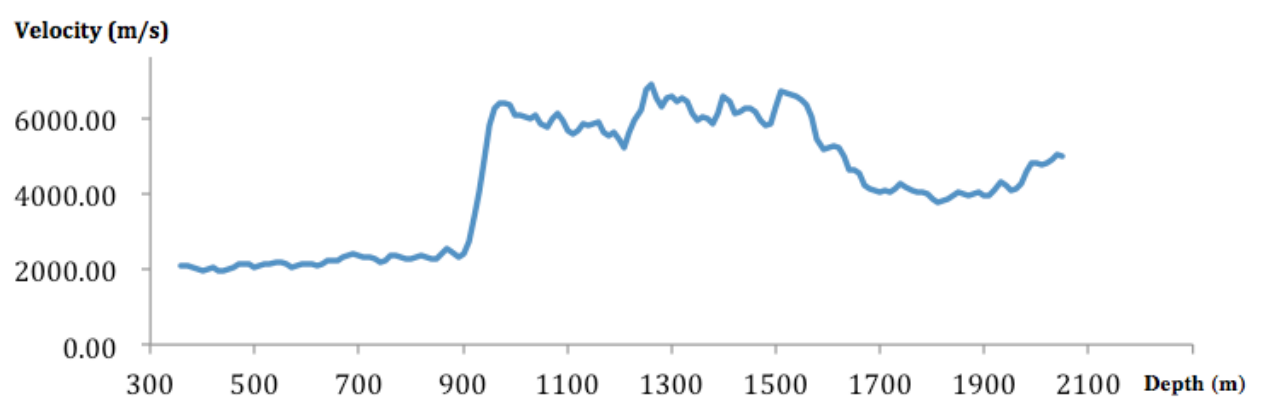

Figure 4.11: P-wave interval velocity estimated from direct P-wave traveltimes.

4. VSP true amplitude recovery

VSP data is similar as surface seismic data in that it suffers from amplitude loss 
due to spherical divergence and inelastic attenuation. One difference is that VSP data generally only travels half of the distance relative to surface data. True amplitude recovery applies a time and possible space variant gain function to traces to compensate for loss of amplitude due to wavefront spreading and attenuation. Figure 4.12 shows the VSP data before (right) and after (left) true amplitude recovery. The constant $d B / \sec$ correction is 6 .

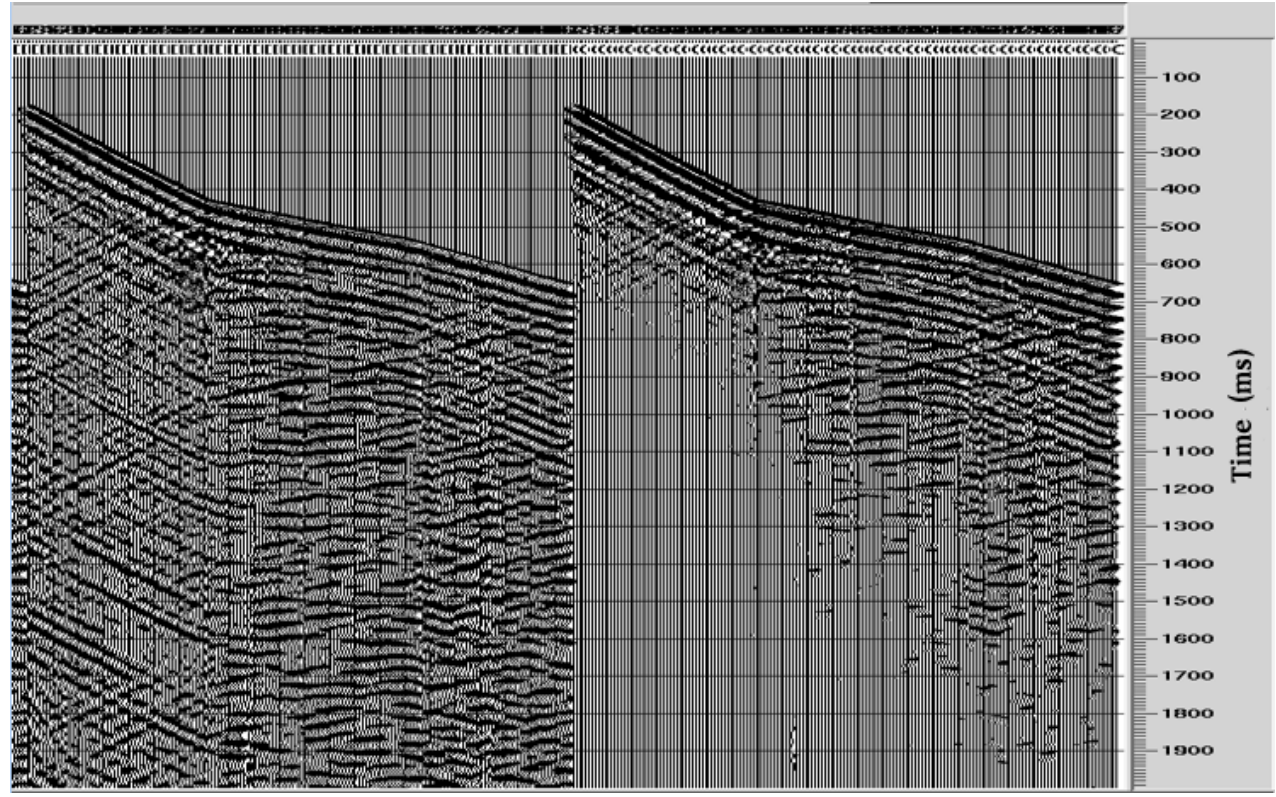

Figure 4.12: The vertical component before (right) and after (left) true amplitude recovery.

5. VSP wavefield separation

Corridor Stack, VSP-CDP transform and/or migration are the final products for most VSP processing exercises. These products usually consist of only upgoing reflected energy. The downgoing energy must be removed from the total wavefield. It is also necessary to isolate the downgoing energy to aid in 
the deconvolution process. The basic tools for wavefield separation are median filter and FK filter.

Median filter is an effective way for estimating the flattened event amplitudes by computing the median amplitude over a series of traces at constant time samples. If the input data is well flattened and the waveforms are stable, then the median filter should perform well.

FK filter is another effective way to separate the input data into various dip components. For up-down separation, FK filter is used to separate the flattened downgoing energy from the dipping upgoing energy. I use the interactive FK analysis module in ProMAX so that various FK polygons are tested to keep the appropriate upgoing and downgoing waves.
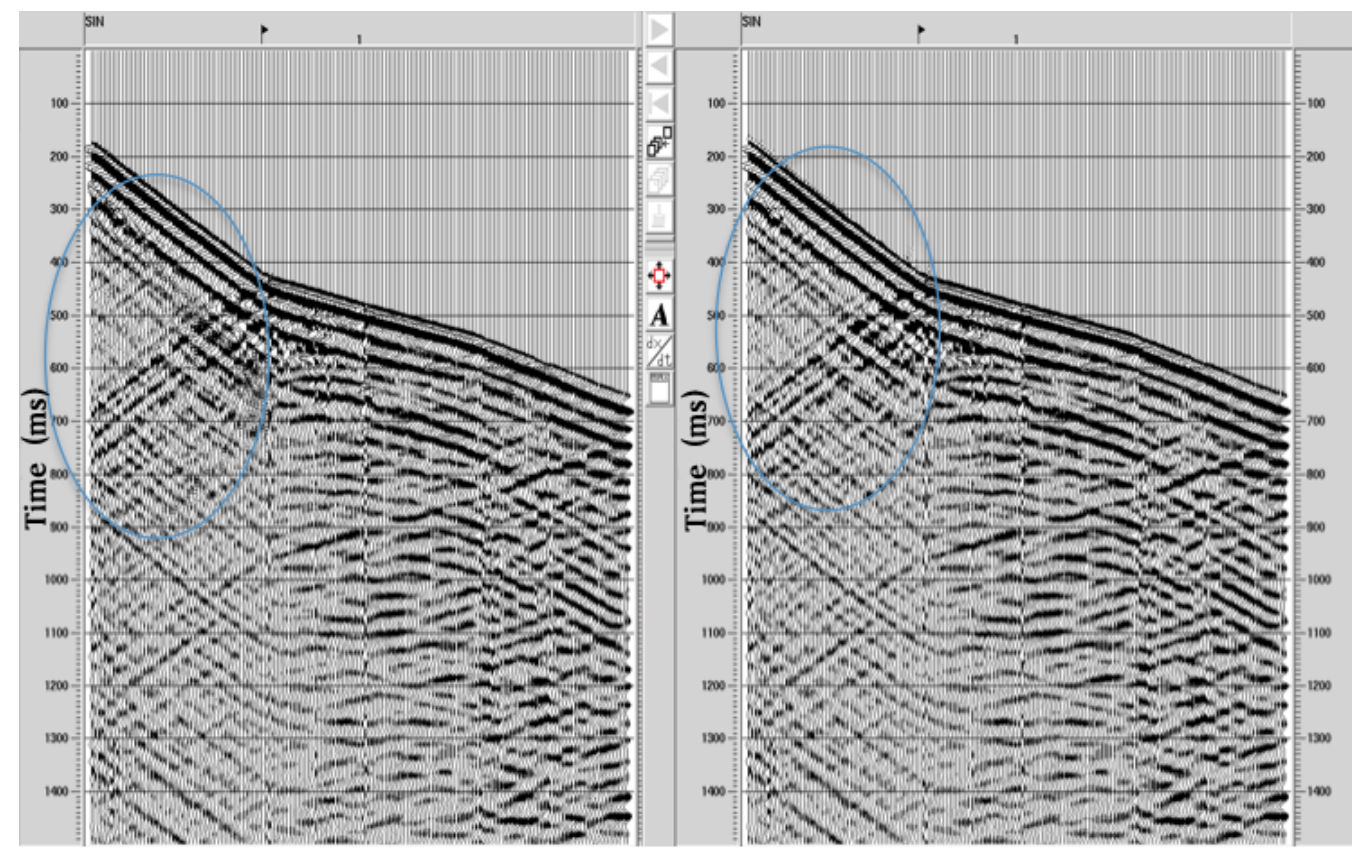

Figure 4.13: The vertical component before (left) and after (right) applying the bandpass filter. 
For separating the field data, I first use a bandpass filter to remove some high-frequency noise, then I try both FK filter and median filter to separate downgoing and upgoing waves. Figure 4.13 shows the results before (left) and after (right) filtering. Figure 4.14a shows the separated downgoing wave from the vertical component of zero-offset VSP via FK filtering. Figure 4.14b shows the separated upgoing waves. Figure 4.15 shows the wavefield separation by using the median filter.

\section{VSP deconvolution}

Deconvolution of VSP data involves the generation of an inverse filter designed to compress an input wavelet to a zero phase wavelet. The input wavelet is commonly extracted from the separated downgoing energy. This filter is then applied to the upgoing data to remove the source signature and output a zero phase wavelet.

The design gate determination is commonly performed to isolate the wavelet from which the inverse filter is designed. This design gate generally starts at zero time, envelopes the first arrivals and progresses in time for a couple of hundred milliseconds. Figure 4.16a shows the flattened VSP downgoing wavefield (left) and upgoing wavefield (right). In the red circle on the downgoing wavefield, it is obvious that multiples are within $100 \mathrm{~ms}-200 \mathrm{~ms}$. In the upgoing wavefield, the multiple reflections are within $800 \mathrm{~ms}-910 \mathrm{~ms}$ (blue circle). Figure 4.16b shows the downgoing wavefield and upgoing wavefield after deconvolution. Compared to Figure 4.16a, it is obvious that multiples are successfully collapsed. 


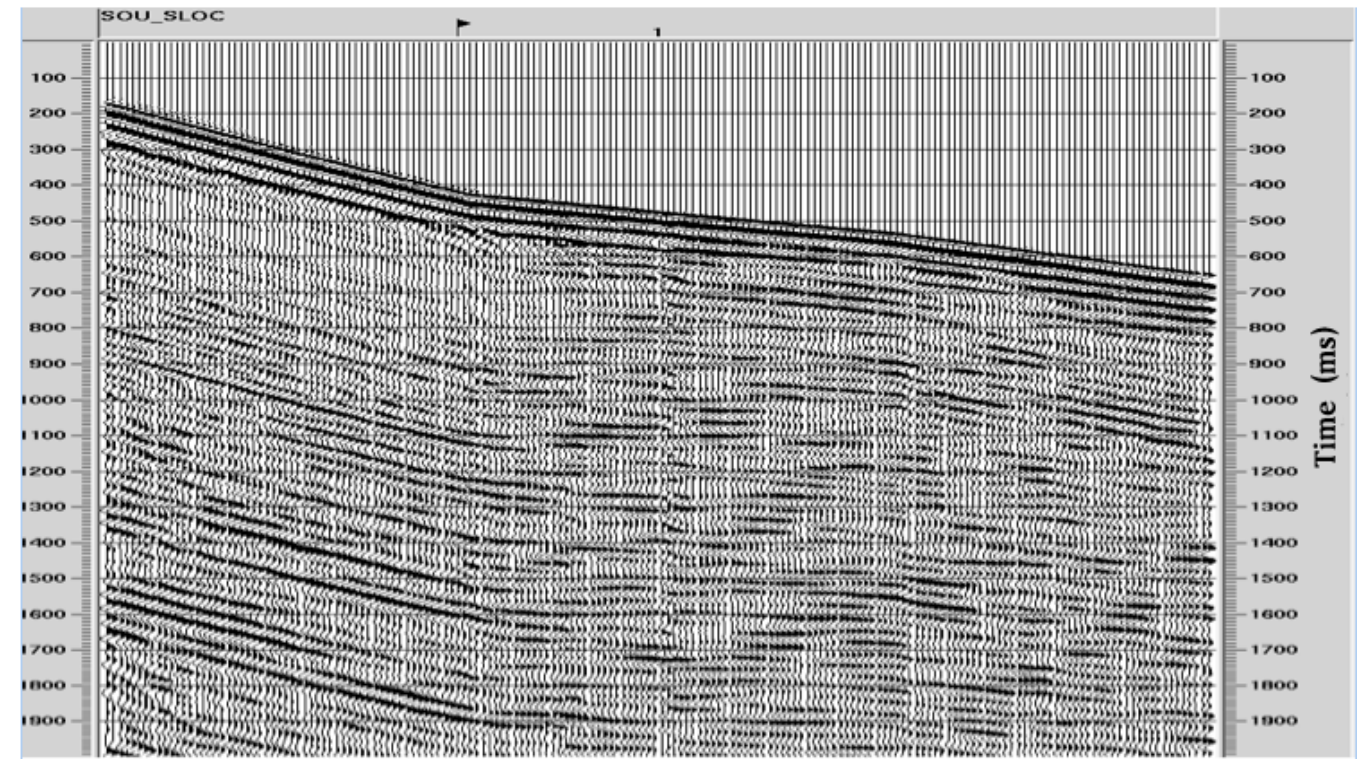

(a)

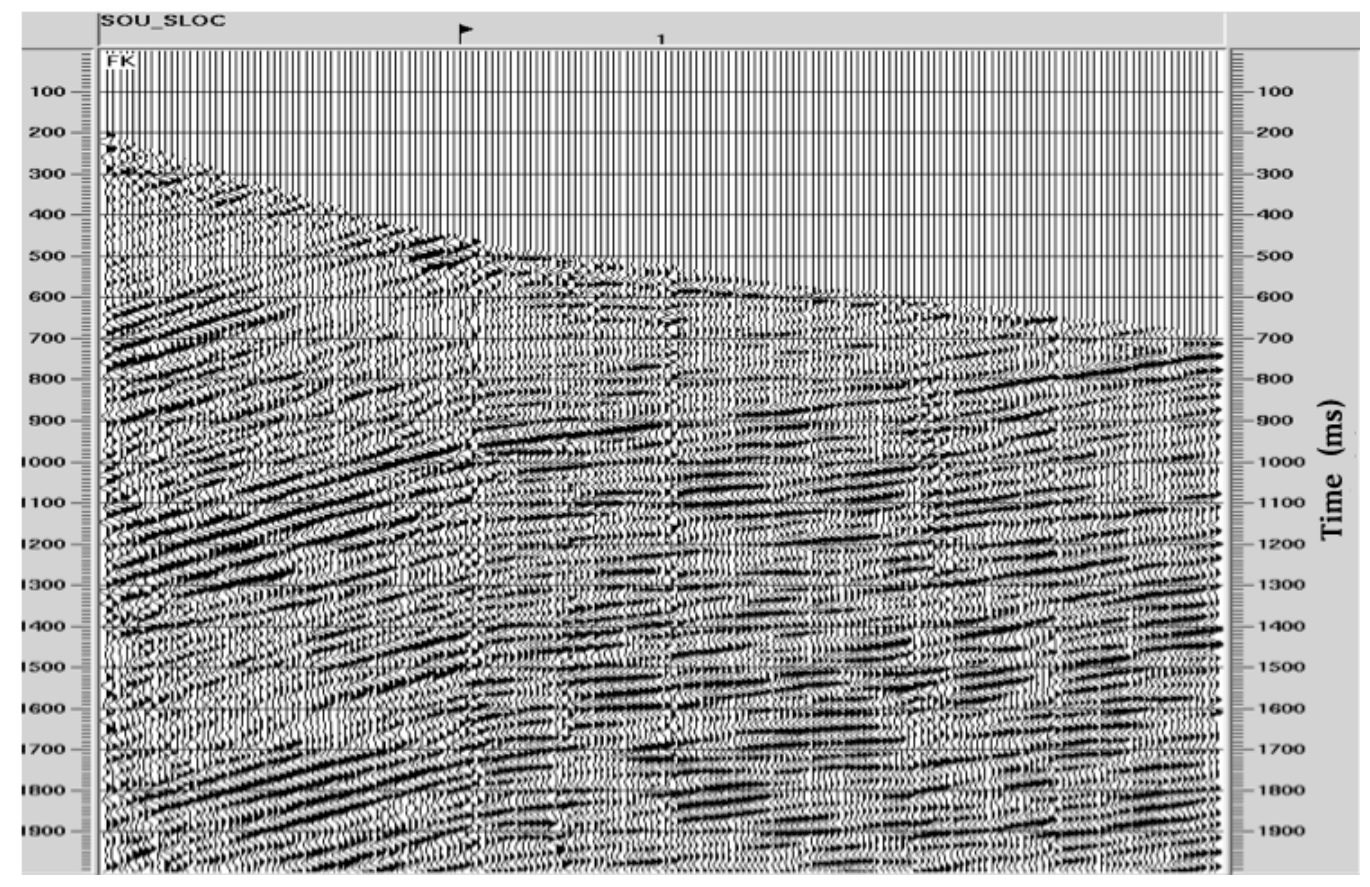

(b)

Figure 4.14: Wavefield separation results using the FK filter. (a) is the separated downgoing waves and (b) is the upgoing waves. 

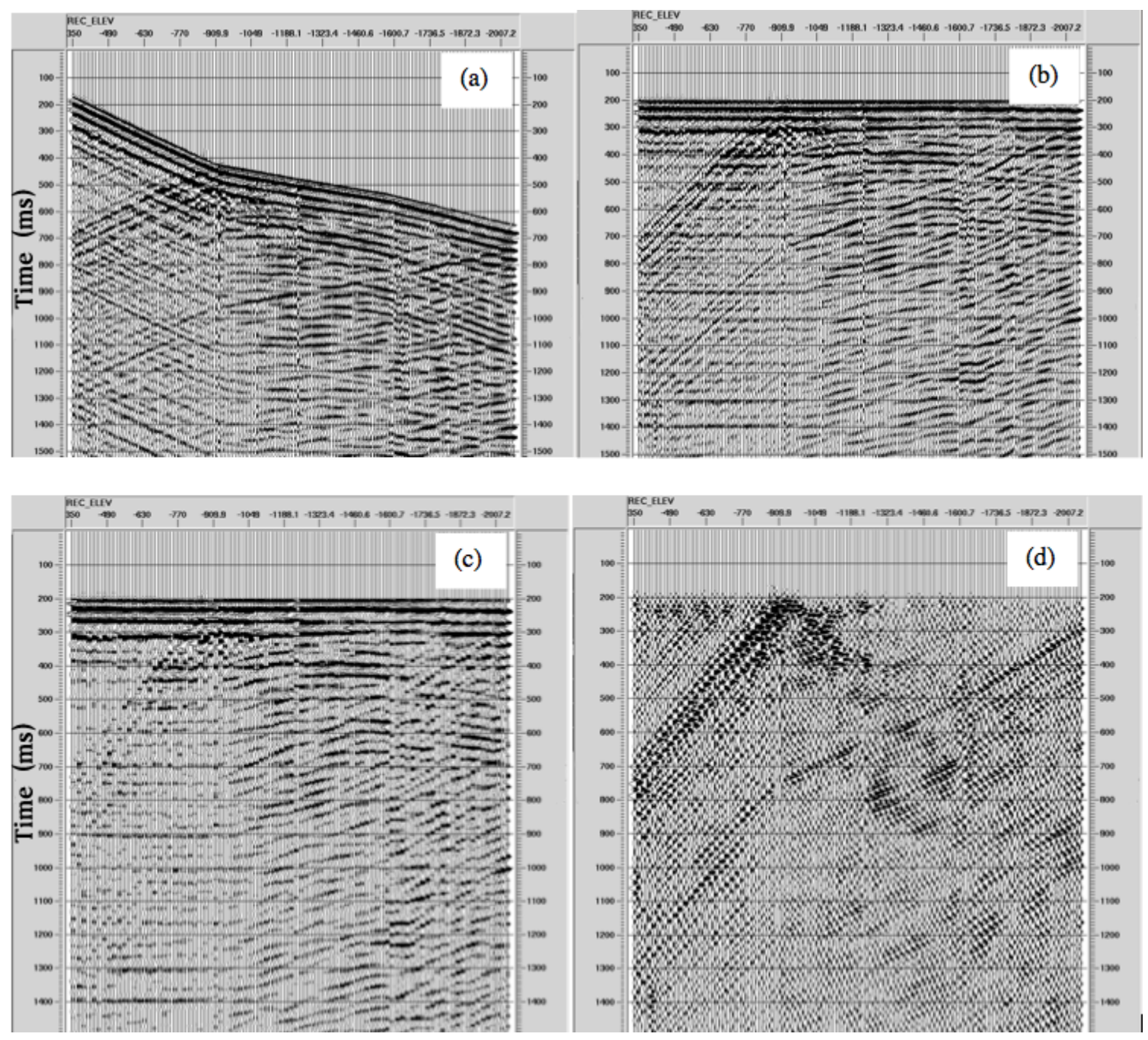

Figure 4.15: Result after applying the median filter. (a) shows all waves; (b) is the flattened waves; (c) is separated downgoing waves; and (d) is the upgoing waves. 


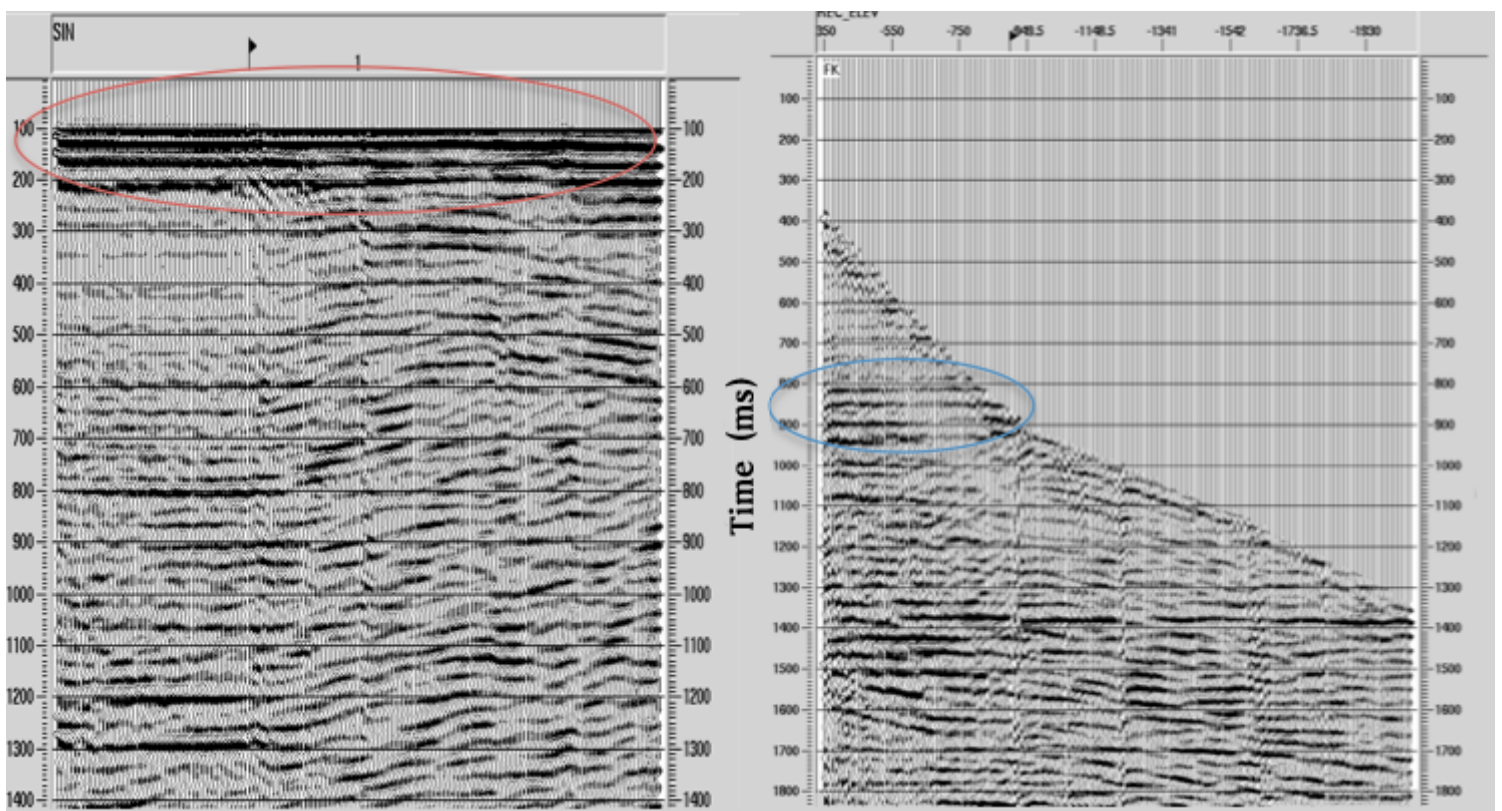

(a)

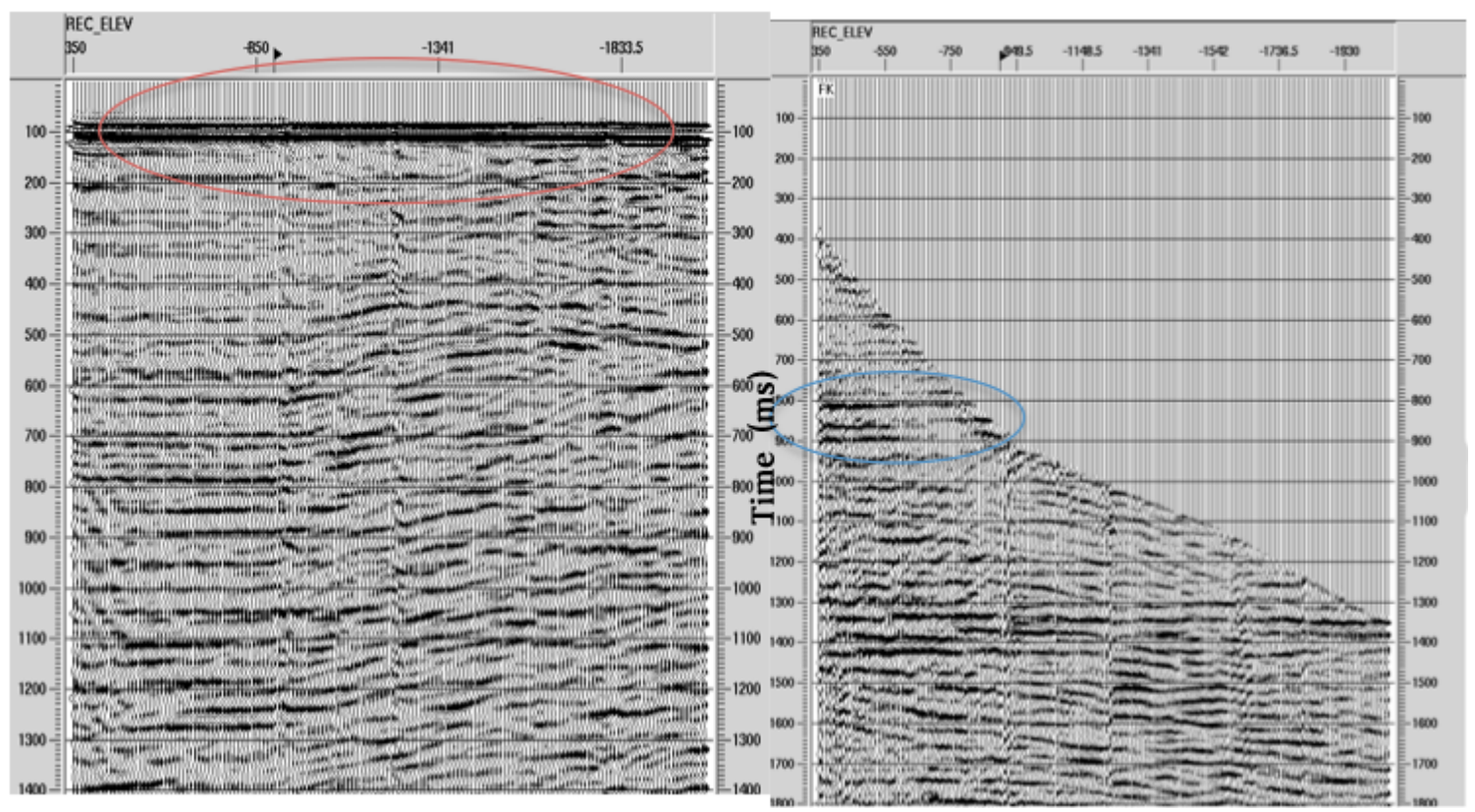

(b)

Figure 4.16: (a) Flattened downgoing waves (left) and upgoing waves (right). (b) Deconvolved downgoing waves (left) and upgoing waves (right). 
7. Corridor mute and corridor stack

Corridor stack is one of the common final products for zero-offset VSP data processing. It can be tied to surface seismic sections or synthetic traces. It can also be used to help processors and interpreters to identify key the geologic horizons at known depths. Besides, the corridor stack can be used to help drillers to predict the deeper depth of the borehole. Figure 4.17 shows the corridor mute and corridor stack of the zero-offset VSP .

8. QC

In order to make sure the processing workflow as well as parameters for processing are suitable, the quality control (QC) is done after each step.

For traveltime picking, I compare the derived velocity from VSP with the acoustic log and Figure 4.18 shows the comparison.

For wavefield separation, I test both FK filter and median filter. Because of the complicate geology in this area, the reflection layers are not flat, and median filter is not effective in this case. So I choose FK filter to separate the upgoing and downgoing wavefield.

For VSP deconvolution, I design different deconvolution operators, and the best one is applied to the downgoing wavefield to collapse multiples.

For corridor stack, I compare the VSP corridor stack with the surface seismic section. Figure 4.19 shows the zero-offset VSP corridor stack overlaid on the surface seismic profile at the well location. It is clear that various formation boundaries are well matched (between $1400 \mathrm{~ms}$ and $1900 \mathrm{~ms}$ ). 


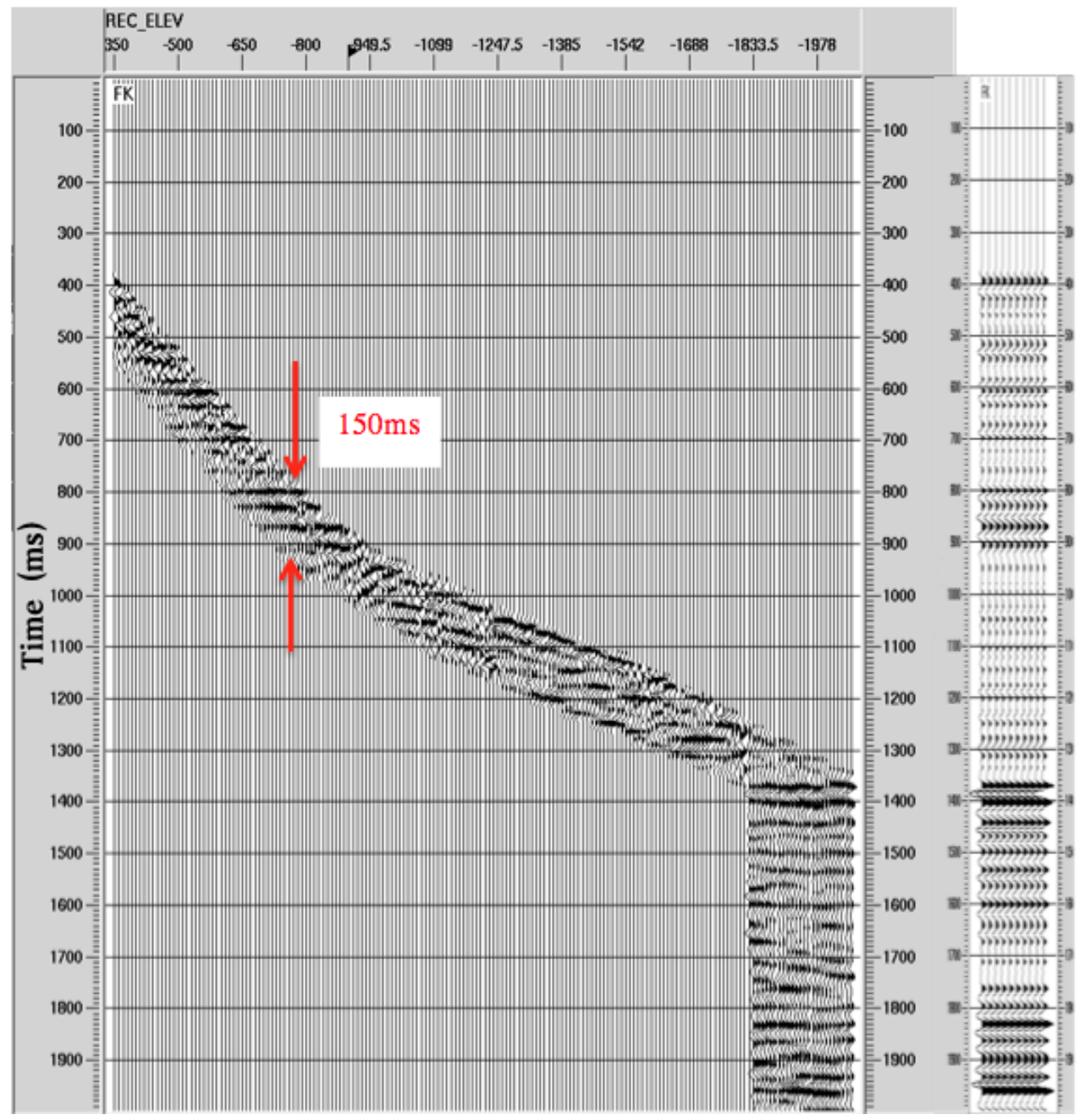

Figure 4.17: Corridor mute (left) and corridor stack (right) of the zero-offset VSP. 


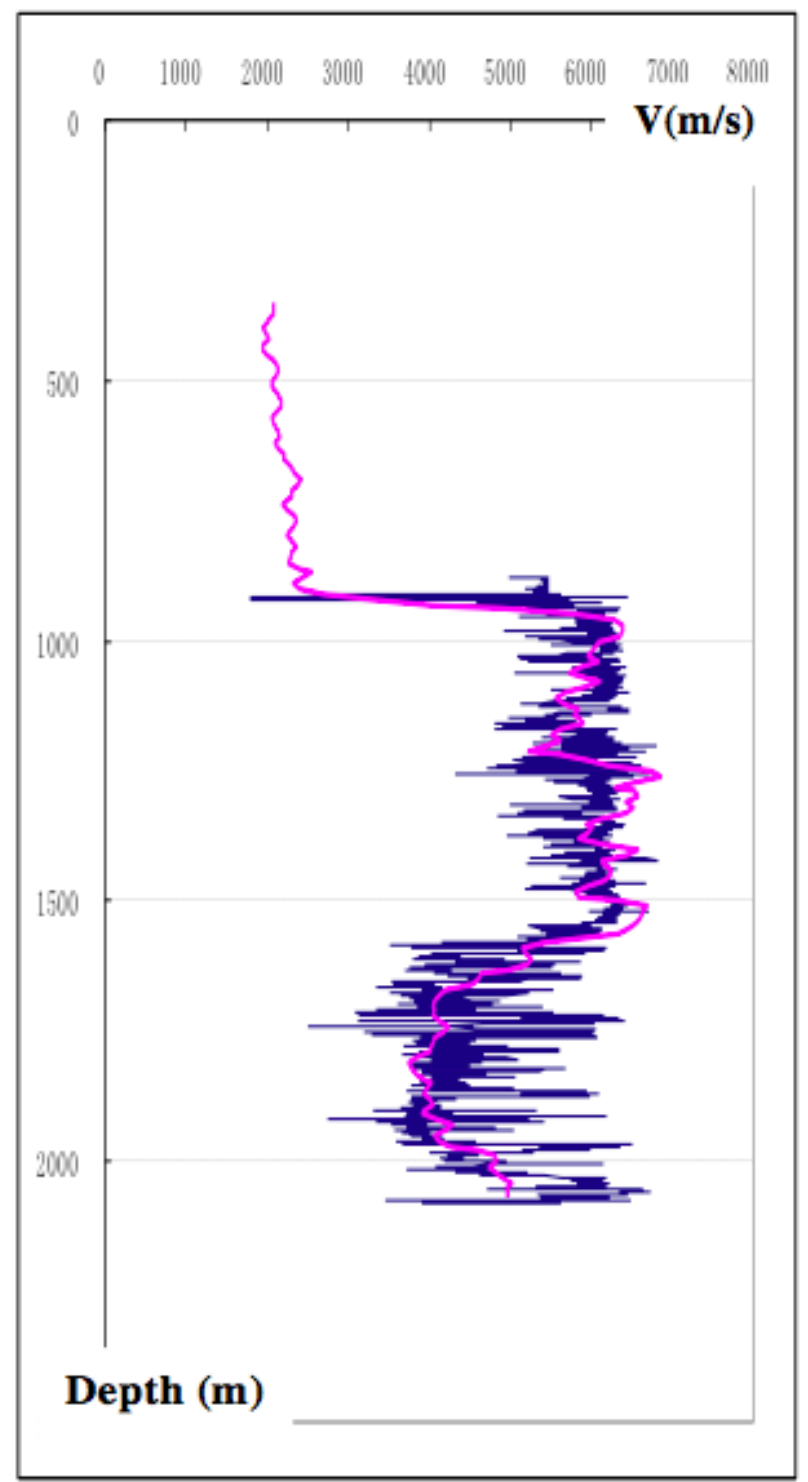

Figure 4.18: Interval velocity (pink) of zero-offset VSP compared with the acoustic velocity (blue). 


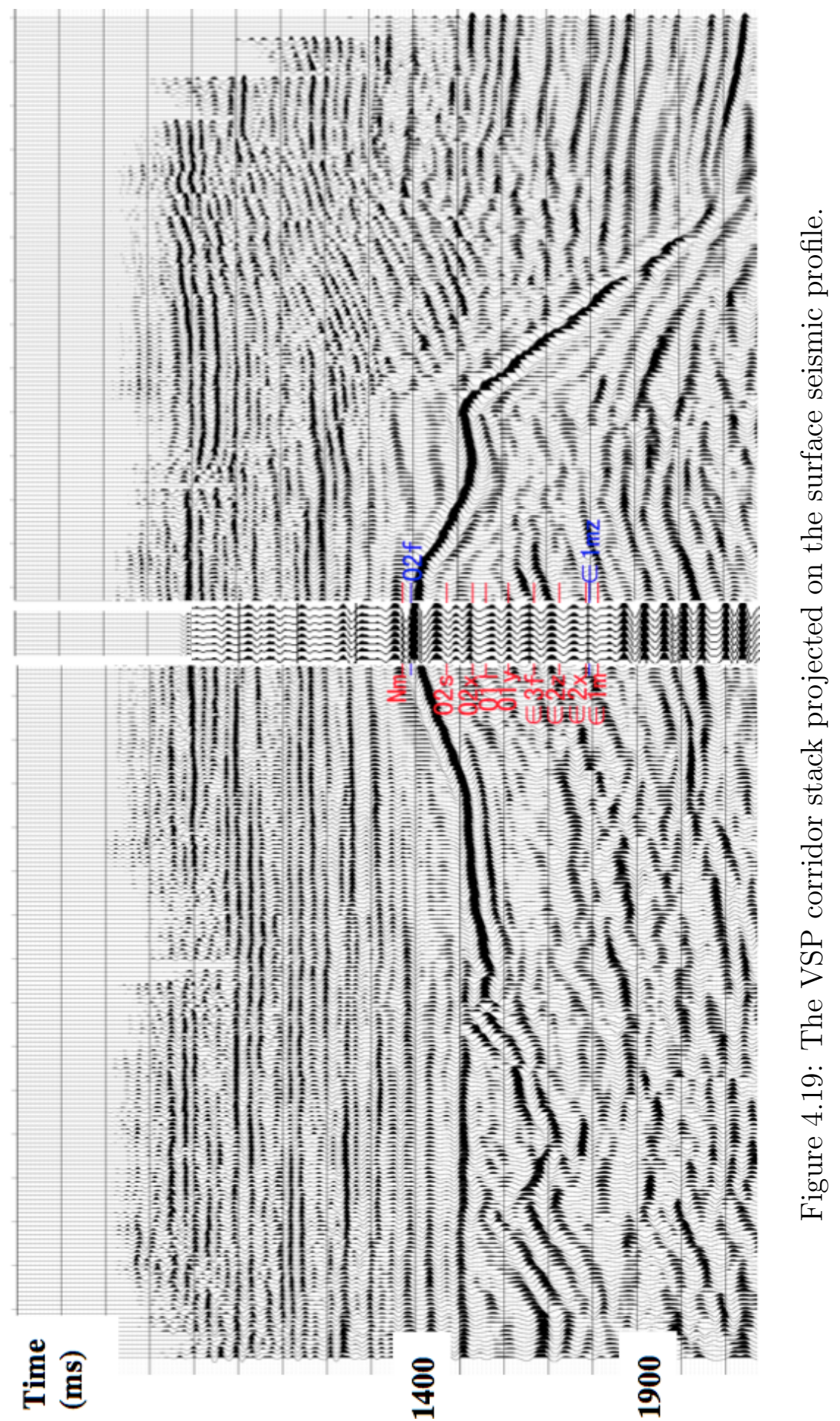




\subsubsection{Offset VSP}

The steps for processing offset VSP are similar to those for zero-offset VSP processing. In this section, I only focus on the $3 \mathrm{C}$ rotation, wavefield separation for offset VSP, VSP deconvolution, and P-wave to converted wave mapping.

\subsubsection{3C rotation}

Most modern downhole seismic recording tools consist of one or more sets of geophones in a string. In this VSP case, two geophones are used for VSP data acquisition. Each of these sets is typically a group of 3C tool ( a vertical geophone and two horizontal geophones) which is oriented perpendicular to one another. For this VSP case, I need to get not only the P-wave image but also the PS-wave image. Therefore, the $3 \mathrm{C}$ rotation is an important step for obtaining converted waves.

Figure 4.20 shows the rotation results. Before $3 \mathrm{C}$ orientation, the converted wave energy in the horizontal component is not strong. After orientation, the converted waves become more obvious.

\subsubsection{Wavefield separation}

For offset VSP wavefield separation, the FK filter is used. Figure 4.21a shows the separated upgoing P-wave from vertical component of the offset VSP. At around $910 \mathrm{~m}$ depth, there is a strong primary reflection follow by multiples. Figure $4.21 \mathrm{~b}$ shows the separated upgoing PS-wave from the horizontal component. The strong 

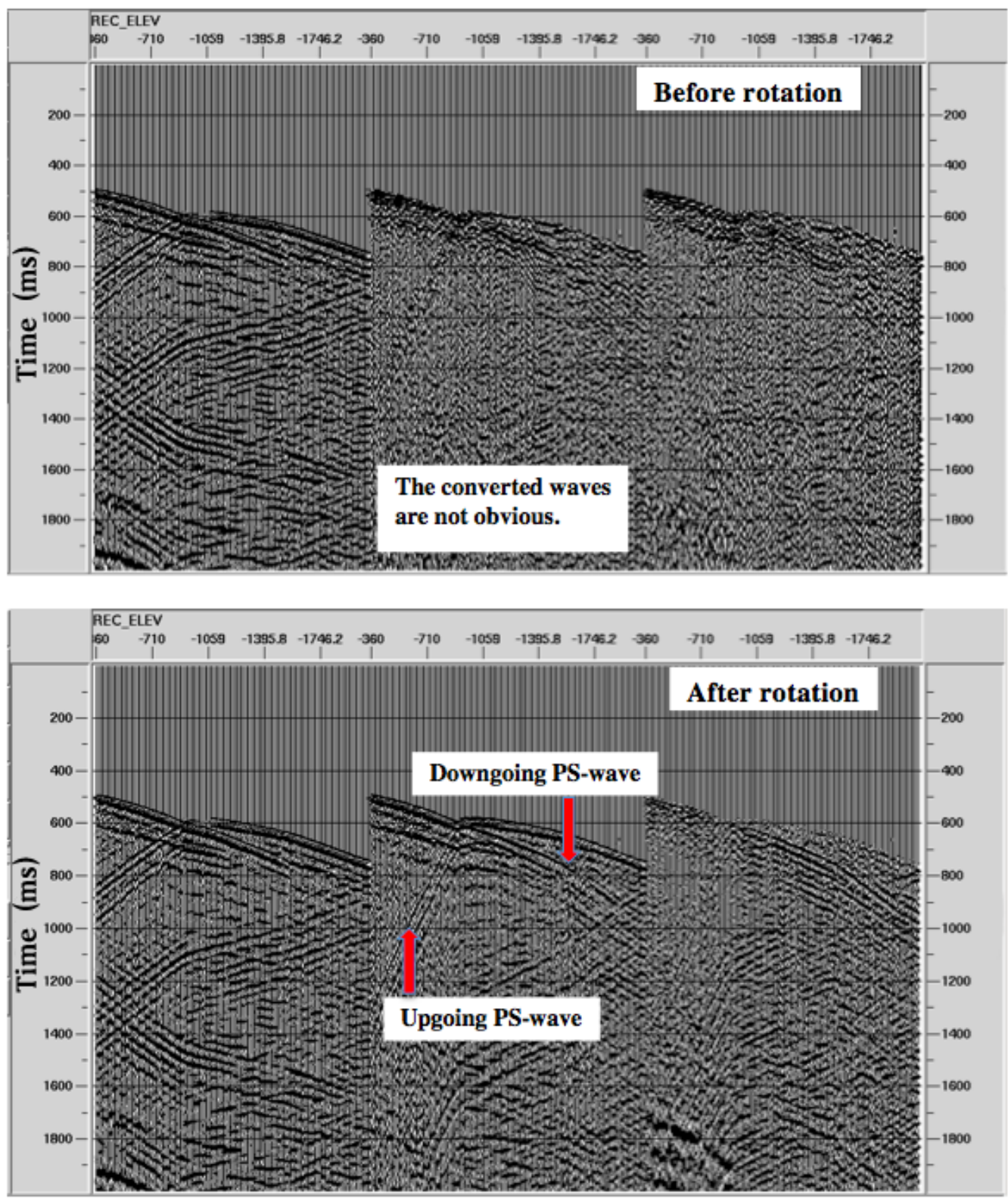

Figure 4.20: 3C offset VSP before and after rotation. 
converted PS-waves are generated from the reflector at around $910 \mathrm{~m}$ depth.

\subsubsection{Deconvolution}

Figure 4.21 shows that the separated wavefields contain multiple reflections; therefore the deconvolution is needed to get rid of these multiples. The steps for offset VSP deconvolution are similar to those with zero-offset VSP deconvolution. First, design a deconvolution operator from the downgoing waves, and use it to shape the downgoing waves. Second, the deconvolution operator is applied to the upgoing P-waves and converted PS-waves. Figure 4.22 shows the result of upgoing P-waves before and after deconvolution. In the red circle, we can see that the multiple reflections have been suppressed. I then apply the same deconvolution operator to the converted waves, and Figure 4.23 shows the deconvolution result of the upgoing PS-waves.

After wavefield deconvolution, the upgoing waves are used for VSP commondepth-point (CDP) mapping and VSP common-conversion-point (CCP) mapping. Before mapping, we need to know the S-wave velocity. In the next section, I discuss how to estimate the S-wave velocity from the 3C VSP field data.

\subsection{S-wave velocity estimation}

In this VSP case, the direct S-waves in the zero-offset VSP are too weak, so it is difficult to pick the first break times of direct S-waves. But the converted PS-waves are strong, and they could possibly be used to estimate the S-wave velocity. The 


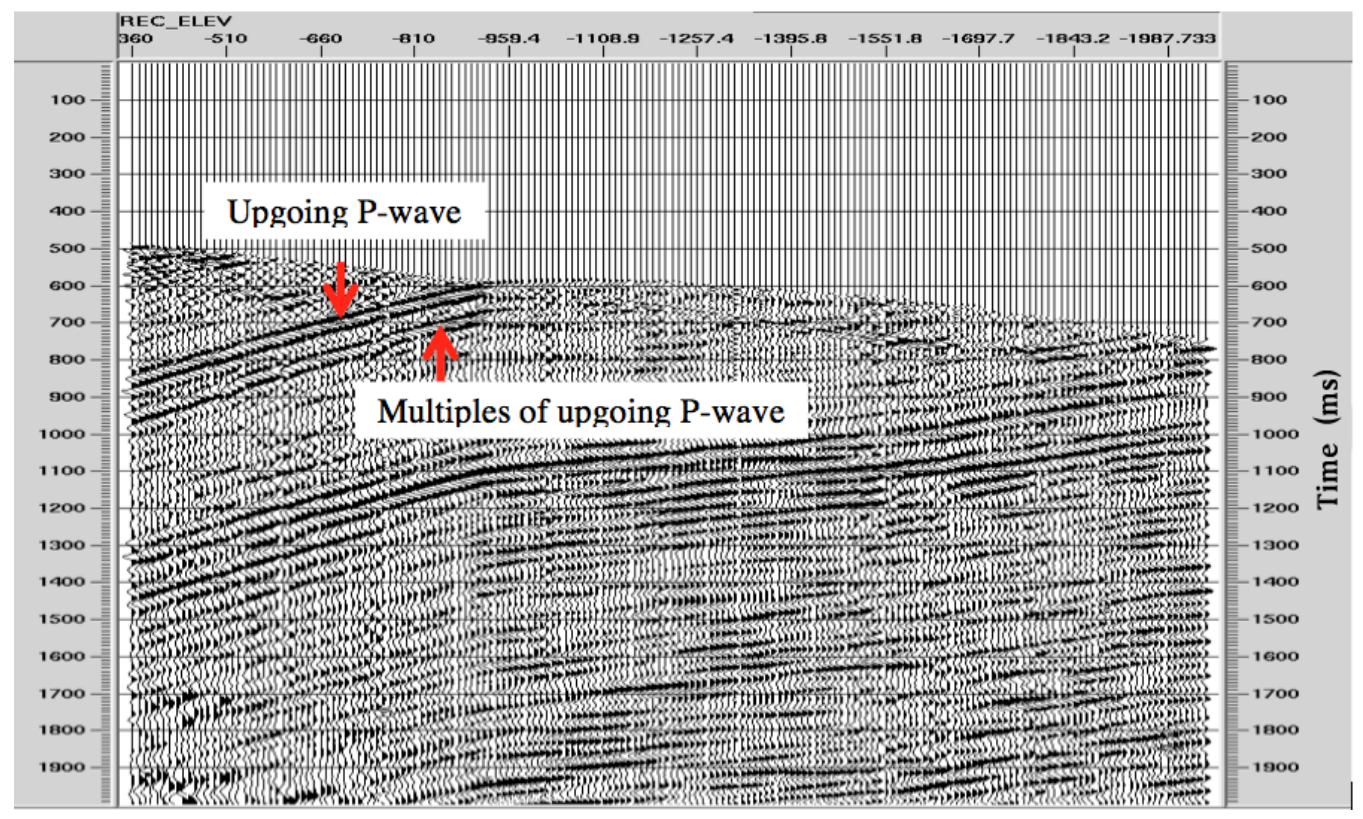

(a)

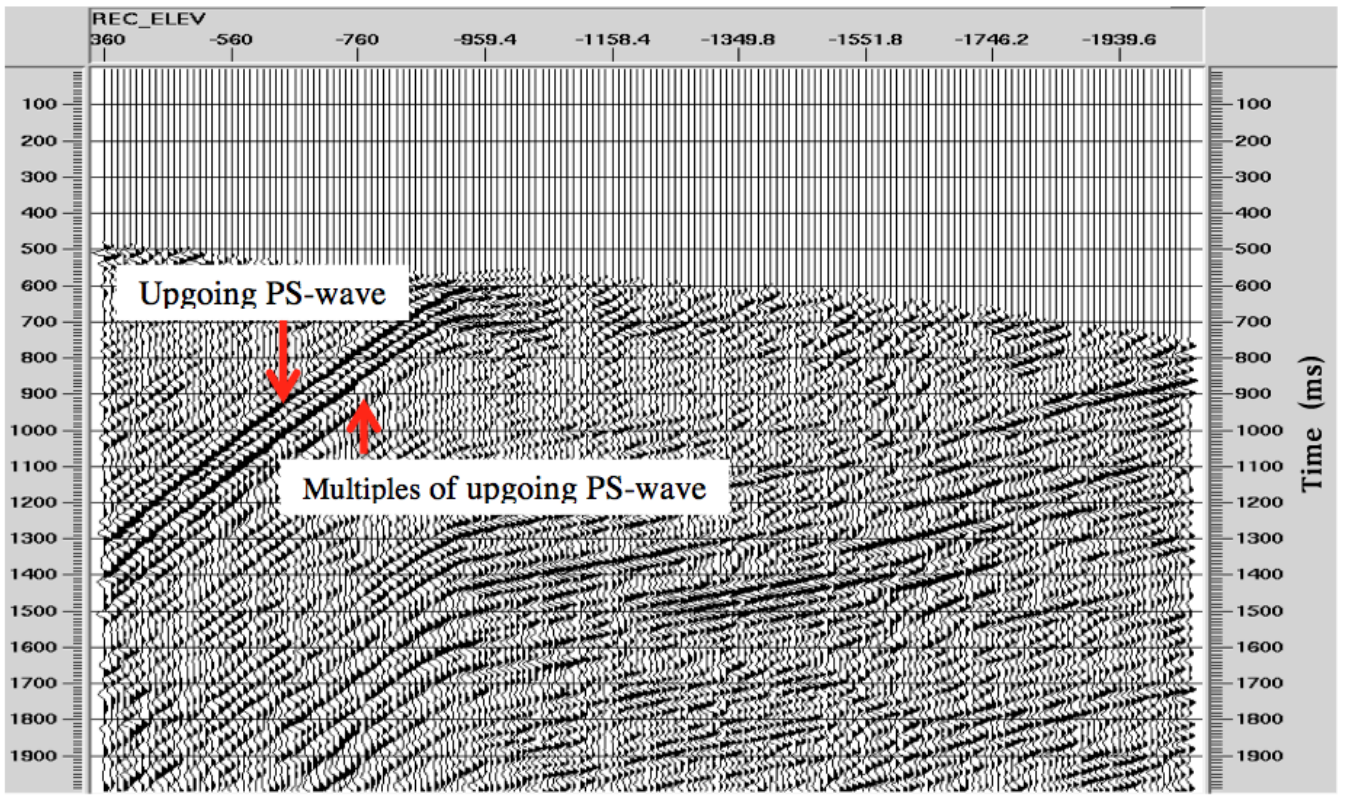

(b)

Figure 4.21: (a) Separated upgoing P-waves. (b) Separated upgoing PS-waves. 


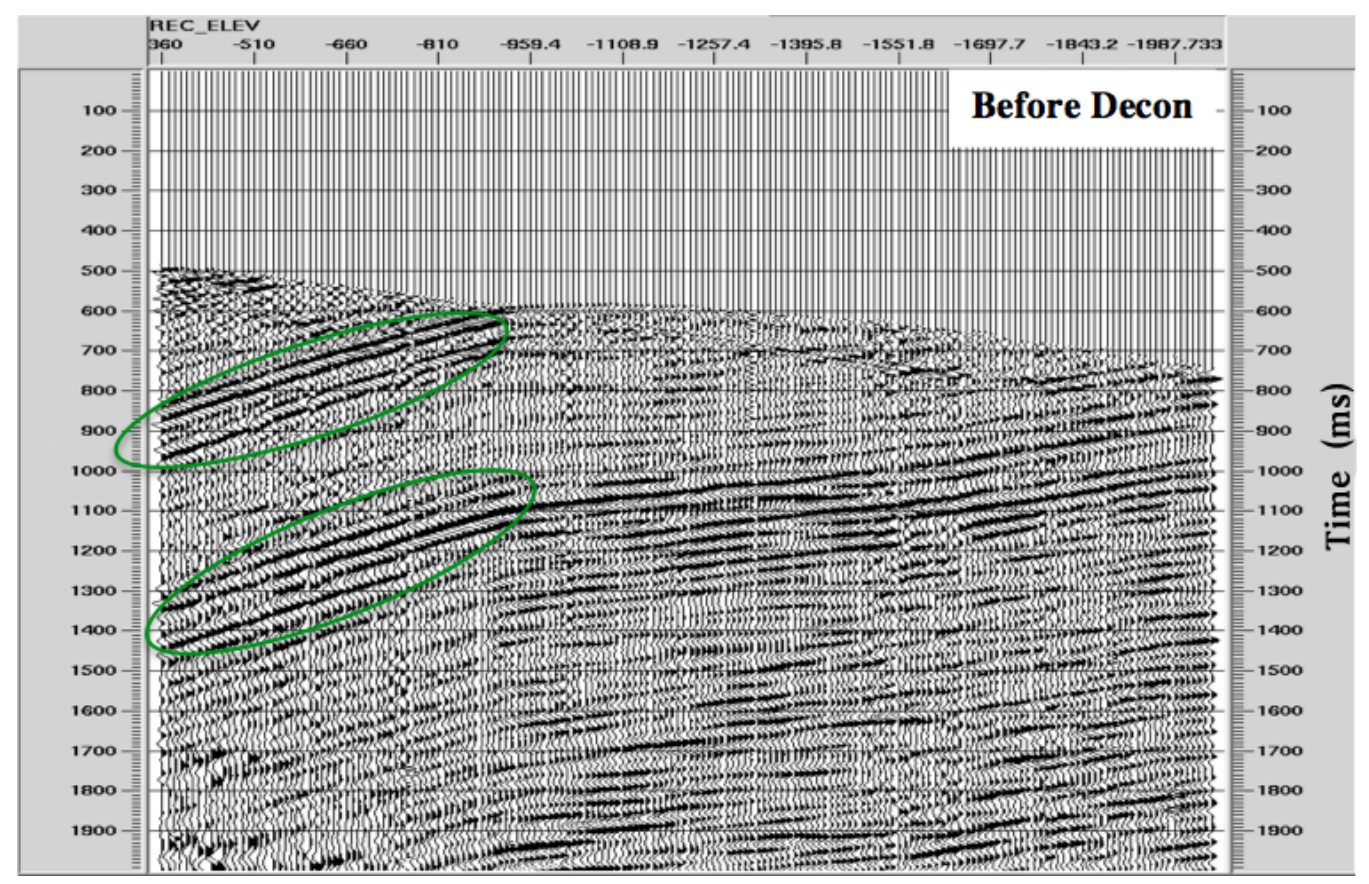

(a)

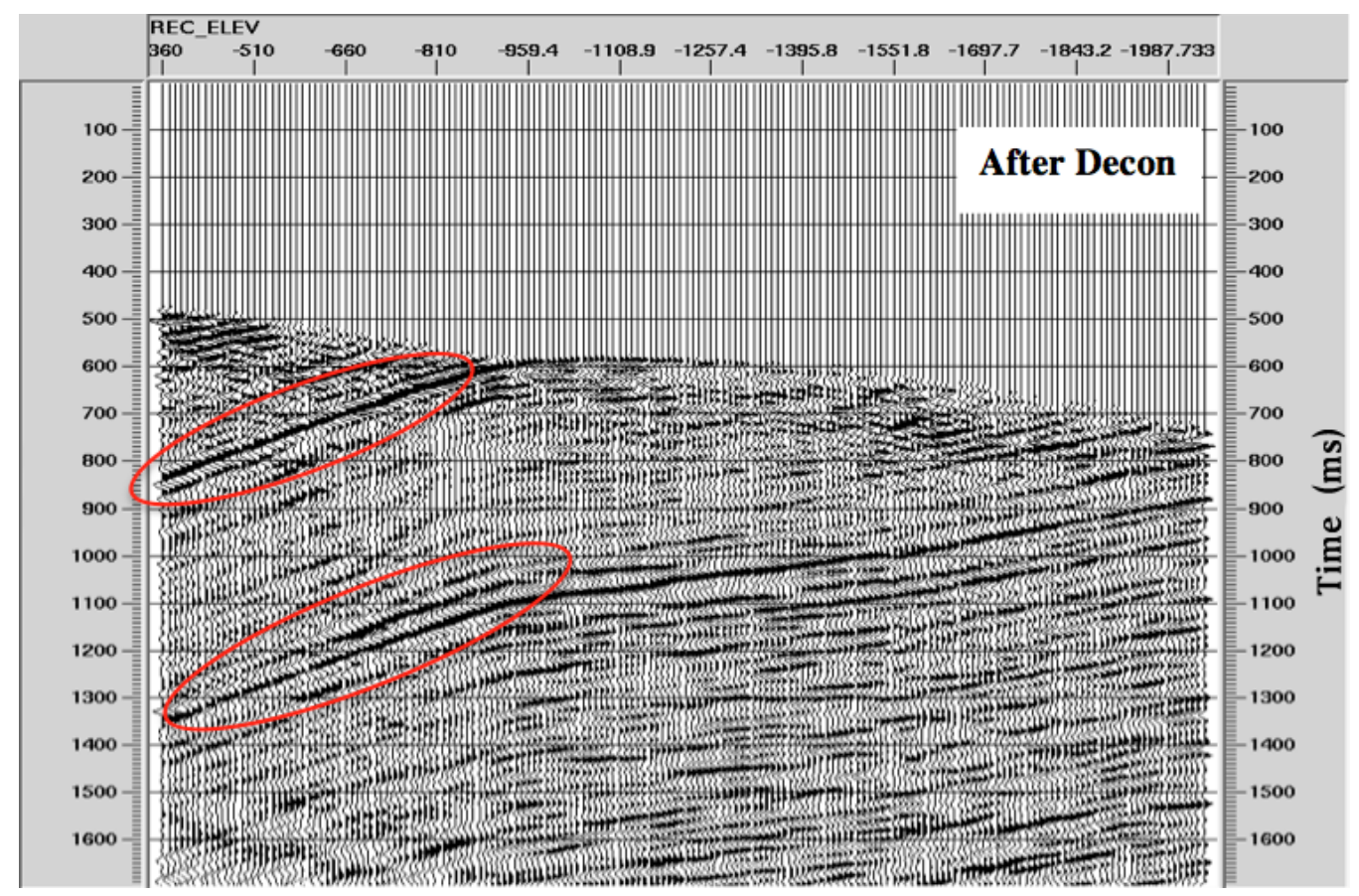

(b)

Figure 4.22: Upgoing P-waves (a) before and (b) after deconvolution. 


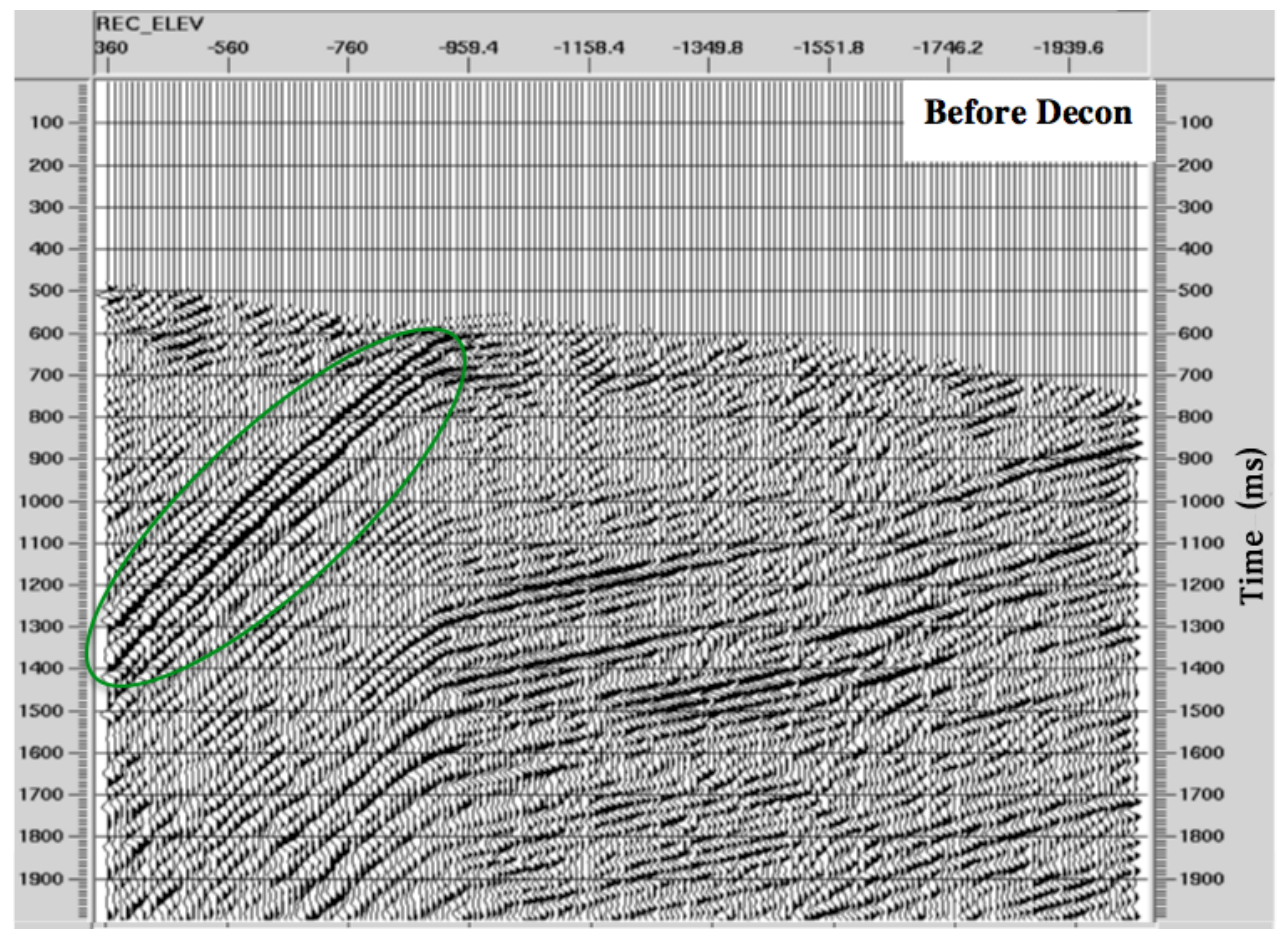

(a)

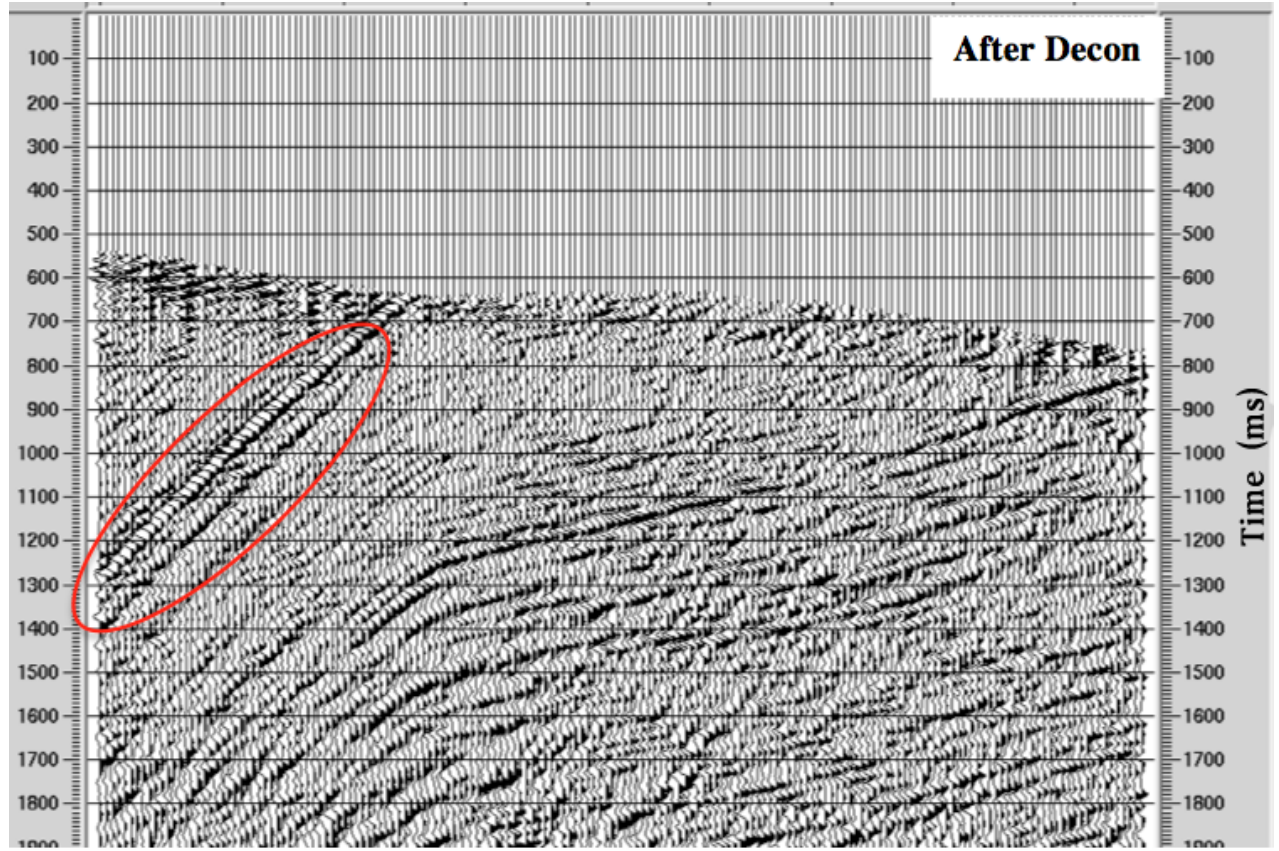

(b)

Figure 4.23: Upgoing PS-waves (a) before and (b) after deconvolution. 
workflow of using converted waves to estimate S-wave velocity is discussed below.

1. Pick converted wave traveltimes

The synthetic test in Chapter 3 showed that traveltime errors will influence the inverted results. To reduce picking errors, I choose the strongest converted PS-wave and pick its traveltimes. Figure 4.24 displays the picked traveltimes (red line) of converted upgoing waves.

2. Build initial velocity model

In this method, we need to provide both $\mathrm{P}$-wave and $\mathrm{S}$-wave velocities to calculate the converted PS-wave traveltimes. The P-wave velocity can be easily estimated from the picked first break times of direct P-wave. For the S-wave velocity, I use Castagna's $V_{p}$ and $V_{s}$ relationship (Castagna et al., 1985) to derive the initial S-wave velocity. Figure 4.25 shows the calculated P-wave velocity (blue curve) and the initial guess of S-wave velocity (red curve).

3. Ray tracing for P-to-S waves

According to the method, the converted wave traveltimes are calculated based on the PS-wave ray paths. Figure 4.26a shows the ray tracing of upgoing converted PS-wave for depth $360 \mathrm{~m}$ to $910 \mathrm{~m}$. The red lines are ray paths of upgoing converted PS-waves, and the black lines are ray paths of downgoing P-waves. Figure 4.26b shows the ray tracing of upgoing converted PS-wave for all depths.

4. Invert S-wave velocity 


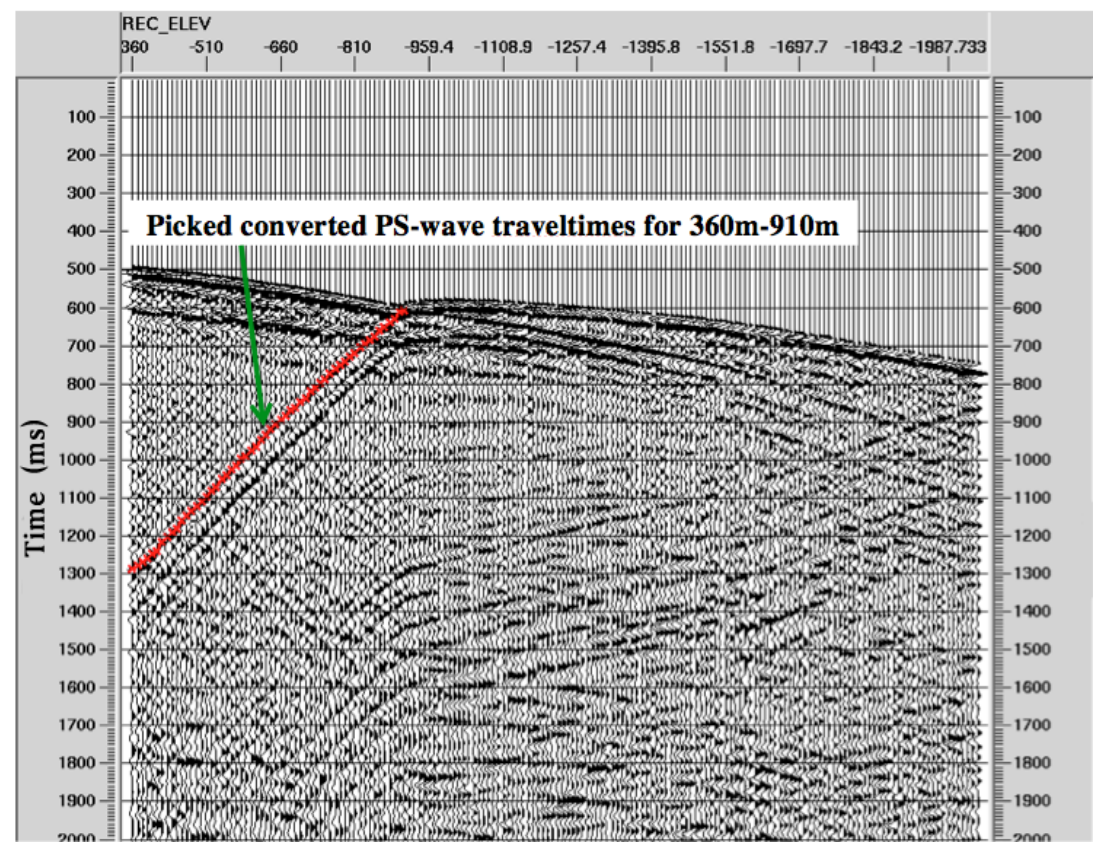

(a)

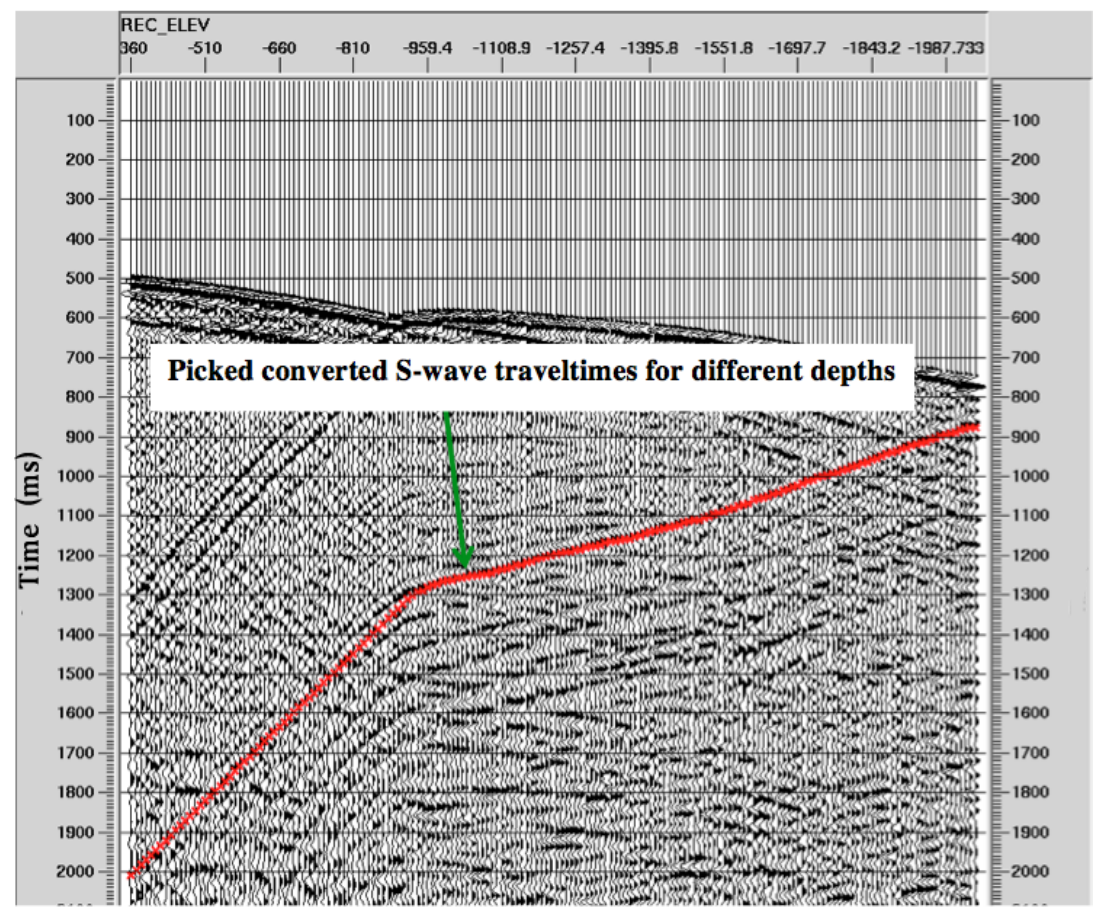

(b)

Figure 4.24: Picked converted PS-wave traveltimes (a) for depth $360 m-910 m$ and (b) for all depths. 


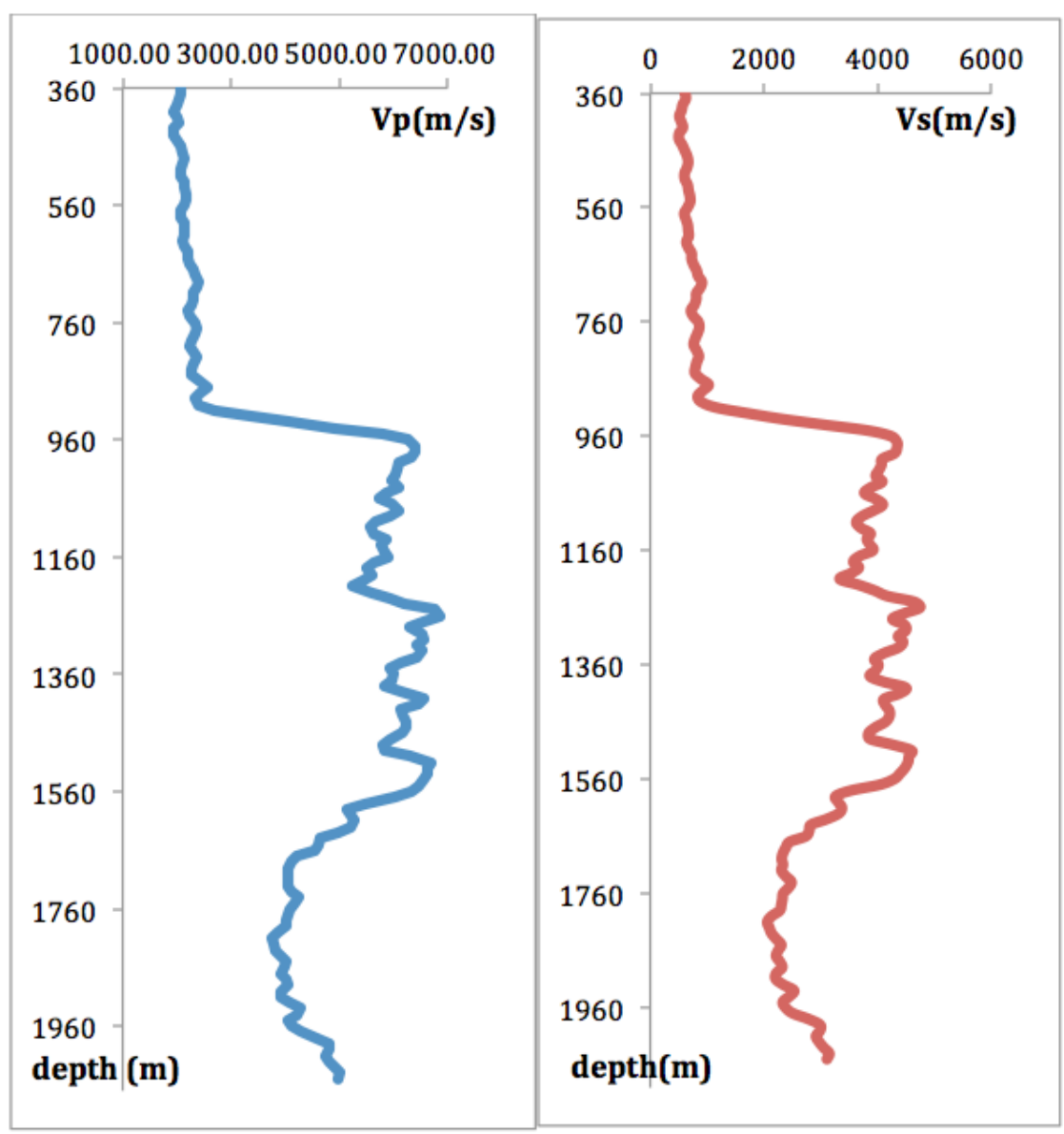

Figure 4.25: P-wave velocity (blue curve) and initial S-wave velocity (red curve).

The inversion steps are listed as below.

- Step 1: Iteration starts from the $n t h$ receiver and S-wave velocity of the $n t h$ layer is estimated first.

- Step 2: Compare the calculated PS-wave traveltimes with the picked PS-wave traveltimes, and update the S-wave velocity iteratively using the traveltime difference.

- Step 3: When the traveltime difference is less than a given criterion, stop the 


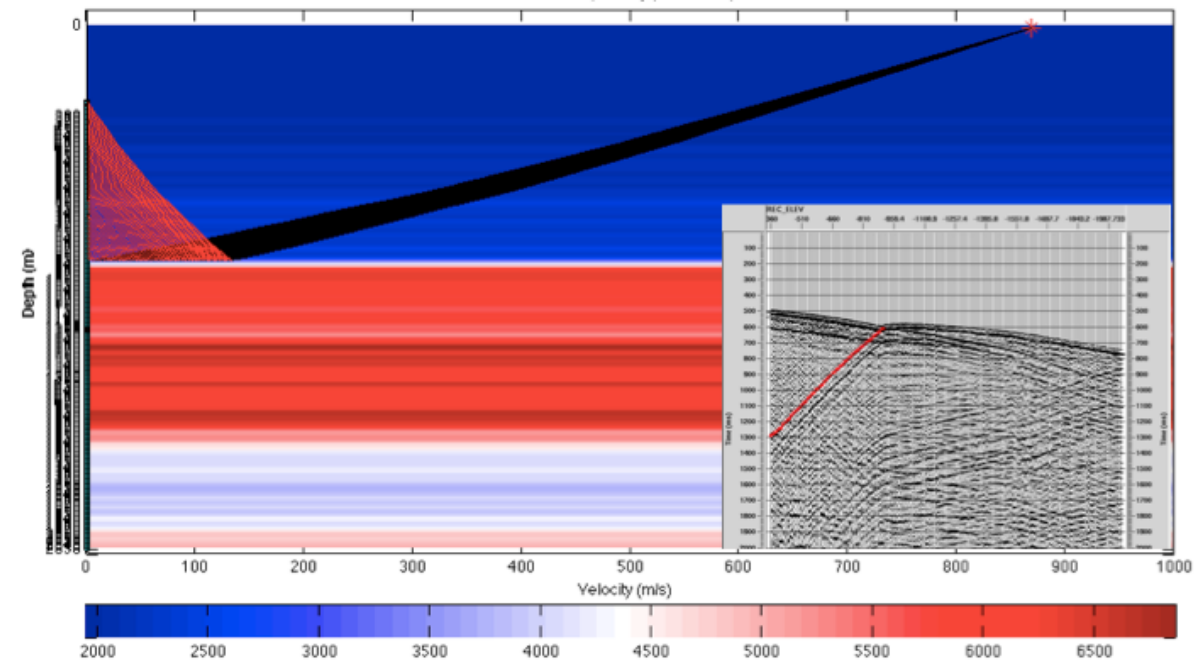

(a)

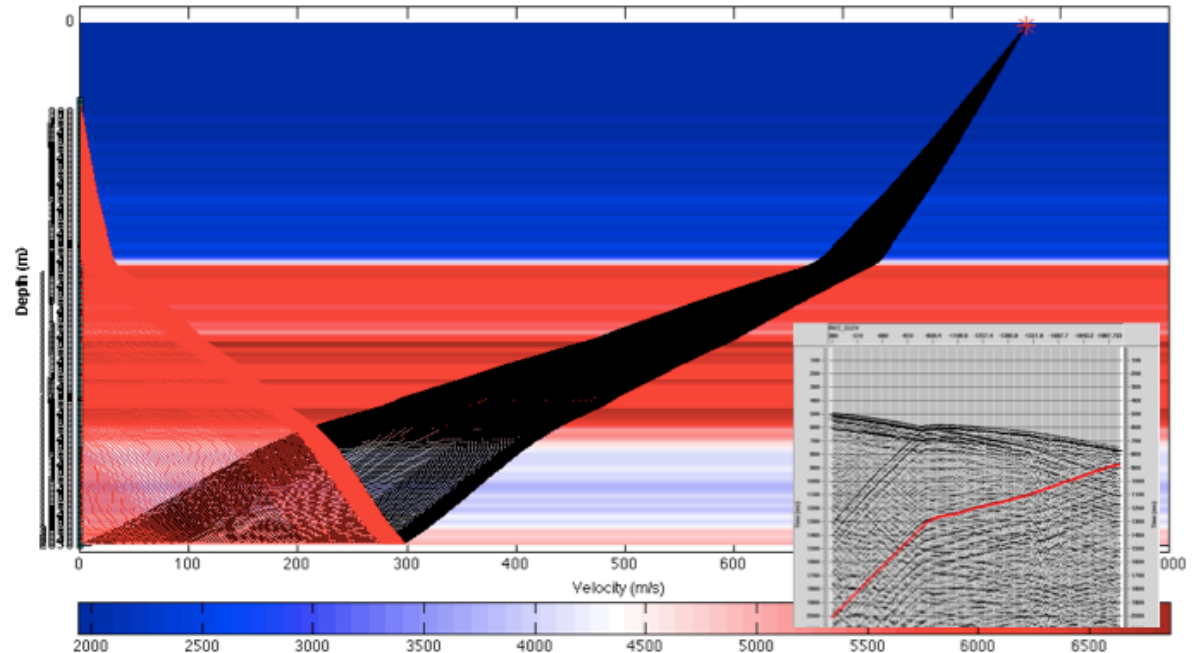

(b)

Figure 4.26: Ray tracing for converted PS-waves (a) for depth $360 m-910 m$ and (b) for all depths. 
iteration and save the current result as the $n t h$ layer S-wave velocity.

- Step 4: Go back to step 1, calculate the PS-wave traveltimes of the $(n-1) t h$ receiver using the $n t h$ layer S-wave velocity obtained from previous iteration. Once all receivers are iterated, the S-wave velocity for all depths is achieved.

\section{Inversion results}

Figure 4.27 a shows the inverted S-wave velocity from depth $360 \mathrm{~m}$ to $910 \mathrm{~m}$. The picked traveltimes and PS-wave ray paths are shown on the top left and top right of the figure. Figure $4.27 \mathrm{~b}$ shows the inverted results for all depths. The picked traveltimes and PS-wave ray paths are are also displayed in the same

figure. Figure 4.28 shows the ratio of inverted $V_{p} / V_{s}$, where $V_{p}$ is estimated from picked traveltimes of direct $\mathrm{P}$-wave; and $V_{s}$ is inverted by using converted wave traveltimes.

\subsection{VSP-CDP mapping and VSP-CCP mapping}

For offset VSP data processing, the final pre-migration product is the VSP-CDP (VSP common-depth-point) image. In multi-component VSP cases, the finial product may include the converted wave VSP-CCP (VSP common conversion point) image. In this section, I show the VSP-CDP mapping of $\mathrm{P}$-waves as well as the VSP-CCP mapping of converted waves.

To interpret the offset VSP data in the form of a vertical cross section, it requires transformation of the reflection data from the depth one-way time domain to the 


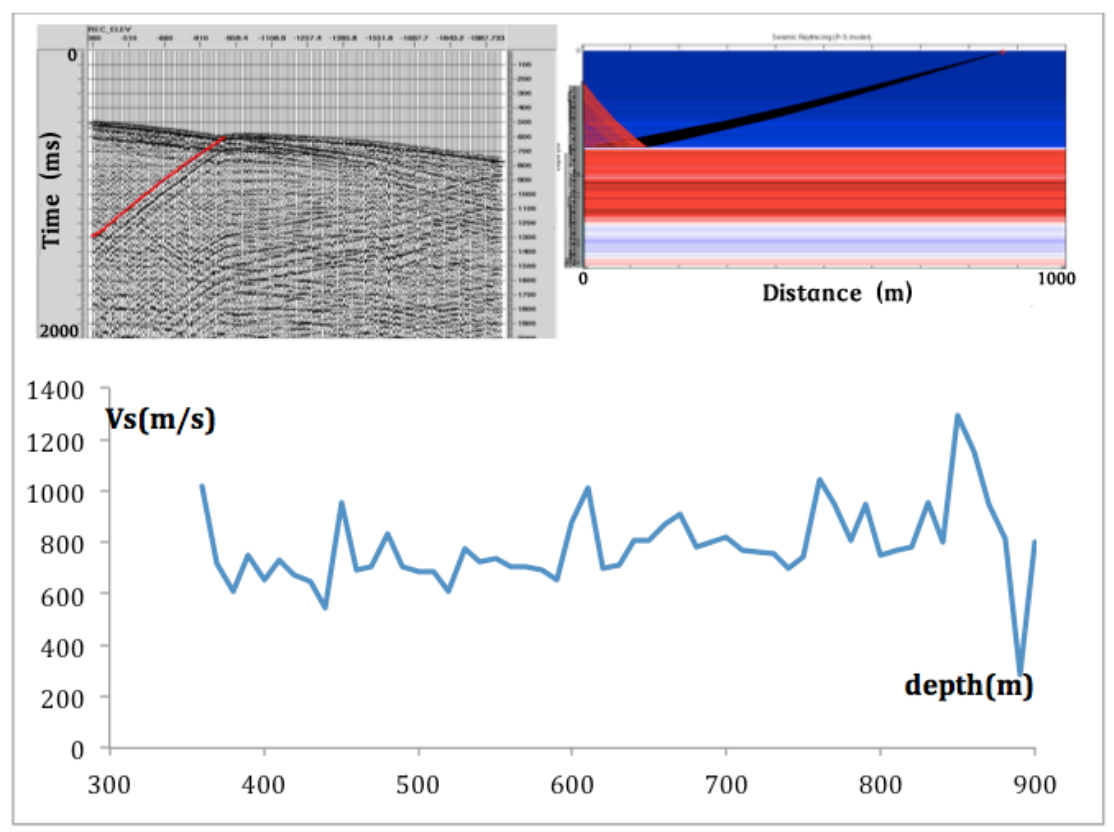

(a)

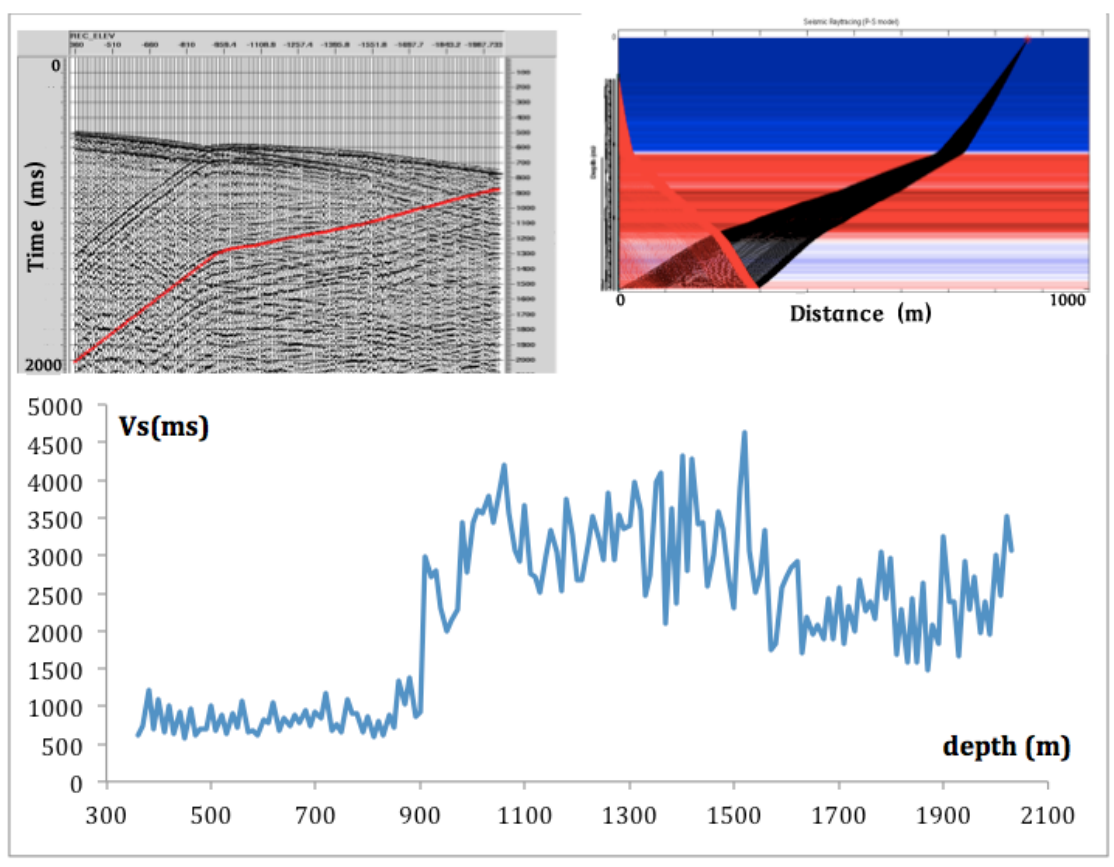

(b)

Figure 4.27: Inverted S-wave velocity (a) for depth $360 m-910 m$ and (b) for all depths. 


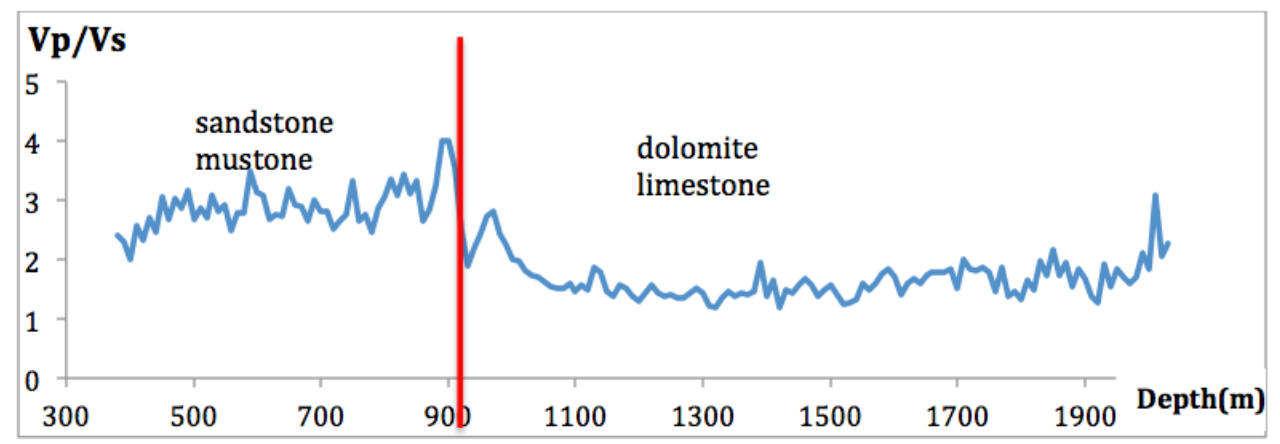

Figure 4.28: The ratio of $V_{p} / V_{s}$.

depth two-way time domain. This can be accomplished by VSP-CDP mapping. The converted PS-wave image can be generated by VSP-CCP mapping. For each sourcereceiver pair, each ray will have a traveltime associated with it as well as a reflection point in depth and offset. By interpolation, each time sample on each trace can be associated with a point in the model. Therefore, the image can be built by mapping the seismic amplitude from the receiver traces to their spatial location in the model.

Converted PS-wave reflection point is different compared with pure P-wave reflection point. Figure 4.29 shows the difference. The P-wave reflection point only relates with $\mathrm{P}$-wave velocity, and the converted point of PS-wave relates with both P-wave velocity and S-wave velocity. Notice that the conversion point of PS-wave is shifted toward the receiver. This is because the S-wave velocity is slower than the P-wave velocity.

Figure 4.30 shows the VSP-CDP map compared to the VSP-CCP map. And Figure 4.31 shows the composite plot consisting of the P-wave velocity, upgoing waves in two-way time, corridor stack, VSP-CDP map and VSP-CCP map. Both the P-wave section and PS-wave section correlate well at the wellhead. And the deep 


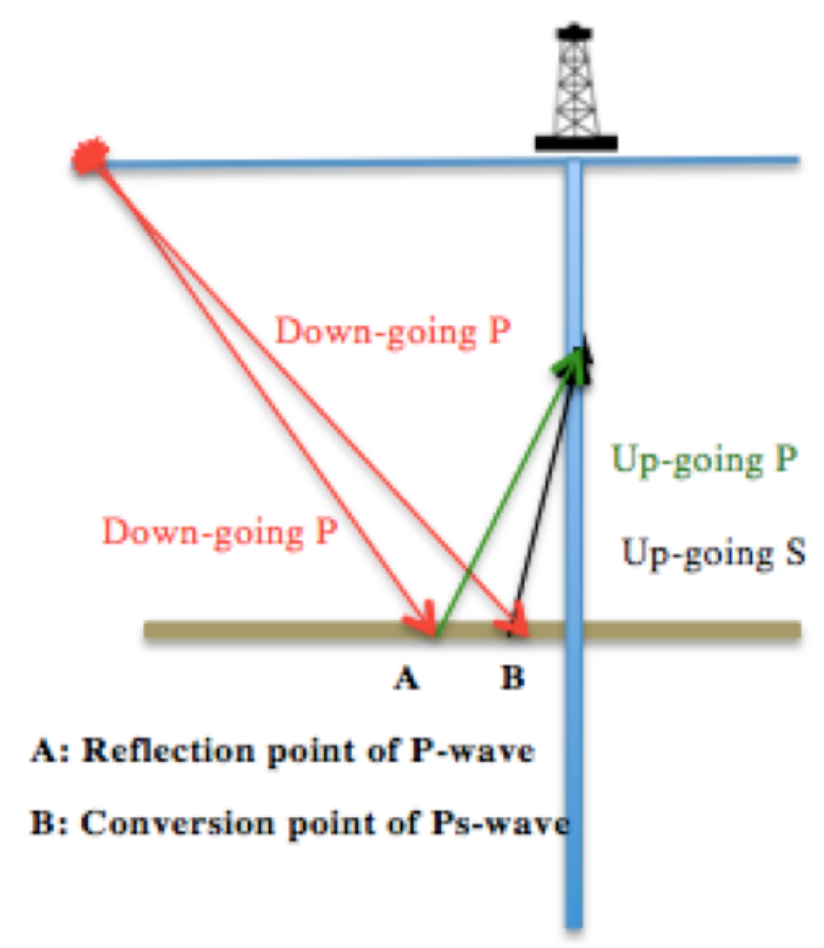

Figure 4.29: Illustration of P-wave reflection point and PS-wave conversion point. section of the PS-wave image is better resolved compared to the P-wave image. It is observed that there is no strict one-to-one correspondence between events of the two sections. This could be due to different responses of P-wave and PS-wave to subsurface elastic properties. After getting the VSP-CDP image and the VSP-CCP image, the 3C VSP data processing work is done.

\subsection{Summary}

The 3C VSP field data is processed first. Then the PS-wave traveltimes are picked from the data and used to estimate the S-wave velocity. Because the true S-wave 
velocity of the studying area is not available, it is not possible to compare the inverted S-wave velocity with the true S-wave velocity. But based on the synthetic tests discussed in Chapter 3), this method can be used to build S-wave velocity and provide a reference value of $V_{p} / V_{s}$ that is useful for VSP-CCP mapping. 


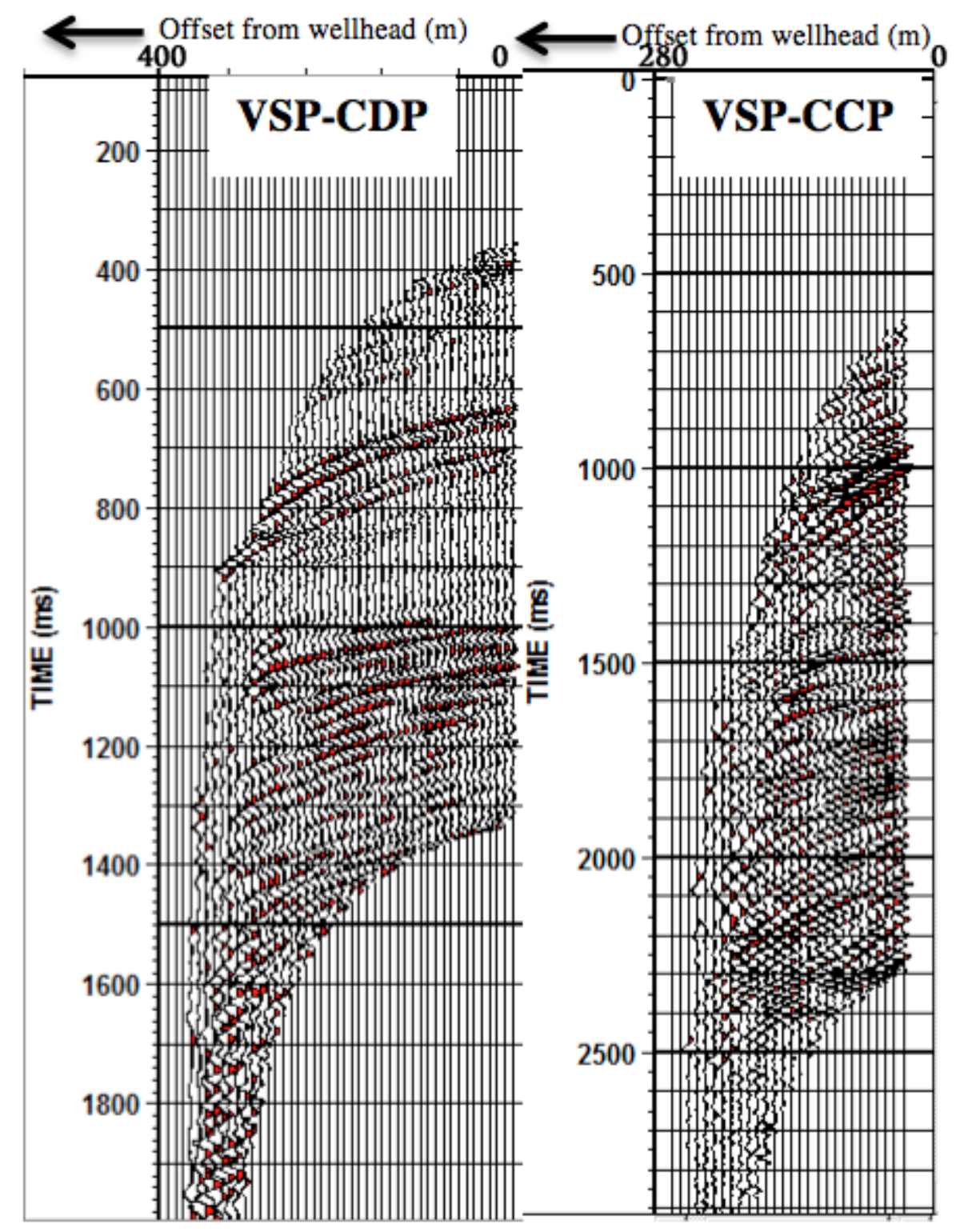

Figure 4.30: VSP-CDP map of P-waves versus VSP-CCP map of converted waves. 


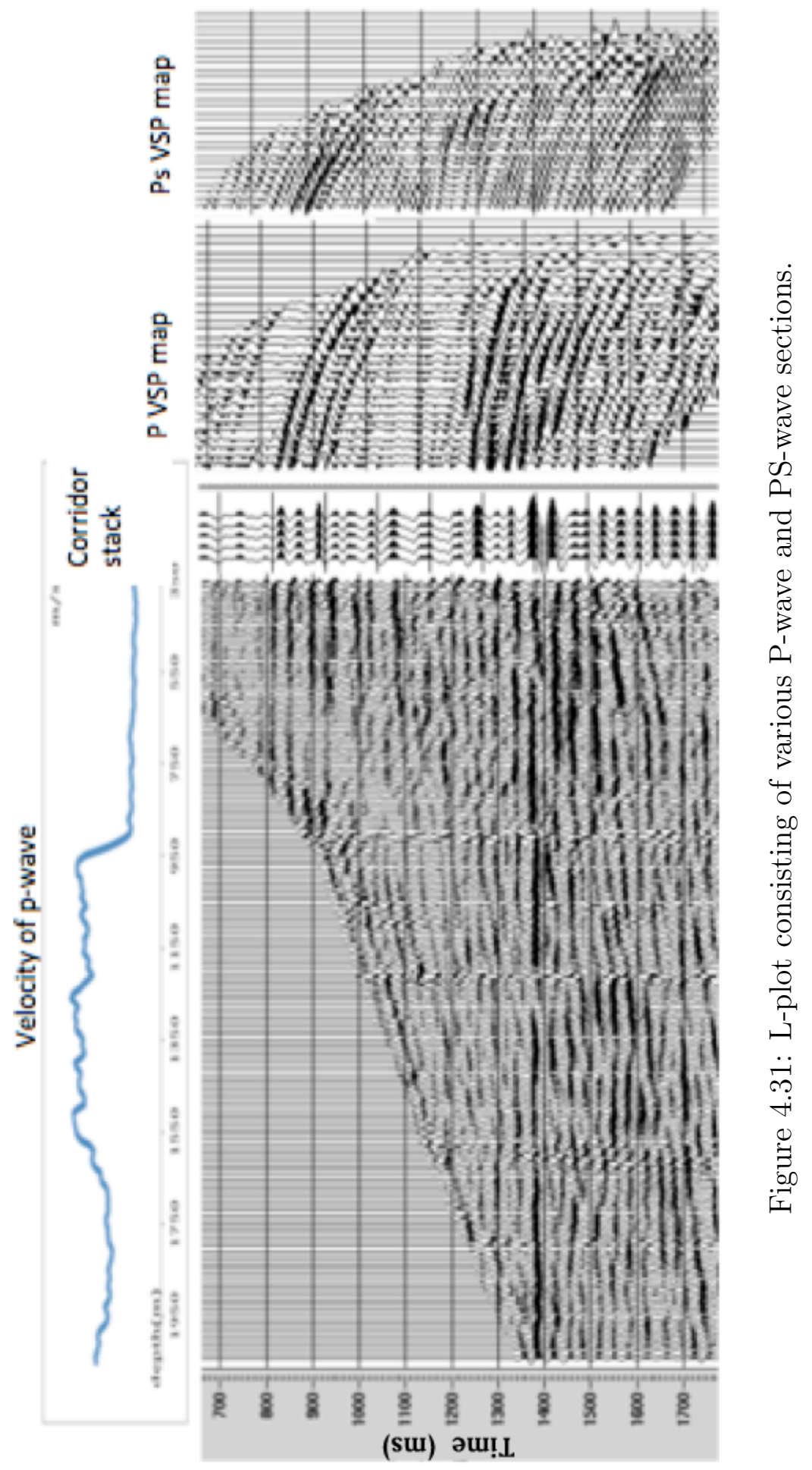




\section{Chapter 5}

\section{Conclusion}

\section{$5.1 \quad$ Summary}

The following conclusions are made based on the discussion of previous chapters.

1. In this thesis, two matrix expressions (equations 2.3 and 2.8) are formulated to calculate converted PS-wave traveltimes.

2. A new S-wave velocity estimation method using converted PS-waves in VSP data is developed. Two synthetic models are tested to validate the method. Results show that this method is effective for S-wave velocity estimation.

3. A practical VSP data processing workflow is demonstrated via the use of the 3C VSP field data. The workflow includes the processing of P-wave, converted wave, zero-offset and offset VSPs. 
4. A novel technology of processing groundroll as zero-offset VSP is presented in Appendix A. With this new technique, groundroll now turns to be a useful signal instead of its old role as noise.

\subsection{Future research work}

The work presented in this thesis can be extended in the following directions.

1. Attenuation tomography using VSP converted waves. The attenuation quality factor Q can be inverted in a similar way as estimating the S-wave velocity.

2. Inversion of VSP converted waves for anisotropy parameters. Transversely isotropic (TI) subsurface models with a vertical (VTI), horizontal (HTI), and tilted (TTI) symmetry axis are also achievable via traveltime-based converted wave inversion techniques. 


\section{References}

Castagna, J., M. Batzle, and R. Eastwood, 1985, Relationships between compressionalwave and shearwave velocities in clastic silicate rocks: Geophysics, 50, 571-581.

Dillon, P. B., H. Ahmed, and T. Roberts, 1988, Migration of mixed-mode VSP wavefields: Geophysical Prospecting, 36, 825-846.

Dillon, P. B., and R. C. Thomson, 1984, Offset source VSP surveys and their image reconstruction: Geophysical Prospecting, 32, 790-811.

Gaiser, J., R. Ward, and J. DiSiena, 1982, Three component vertical seismic profiles: Polarization measurements of P-wave particle motion for velocity analysis: SEG Technical Program Expanded Abstracts, 162-165.

Geis, W., R. Stewart, M. Jones, and P. Katapodis, 1990, Processing, correlating, and interpreting converted shear waves from borehole data in southern Alberta: Geophysics, 55, 660-669.

Hardage, B. A., M. V. DeAngelo, P. E. Murray, and D. Sava, 2011, Multicomponent seismic technology: Society of Exploration Geophysicists.

Hou, A., W. Geng, W. Zhang, W. B., H. Wu, W. Wang, and N. Lei, 2008, Walkaway VSP data imaging using a multilayered anisotropic model: SEG Technical Program 
Expanded Abstracts, 533-537.

Jones, I. F., 2010, Tutorial: Velocity estimation via ray-based tomography: First Break, 28, 45-52.

Li, Y., X. Zhao, R. Zhou, D. Dushman, and P. Janak, 2005, 3C VSP tomography inversion for subsurface P- and S-wave velocity distribution: SEG Technical Program Expanded Abstracts, 2625-2628.

Luo, Y., Q. Liu, Y. Wang, and M. AlFaraj, 2006, Imaging reflection-blind areas using transmitted PS-waves: Geophysics, 71, S241-S250.

Stewart, R., J. Gaiser, R. Brown, and D. Lawton, 2002, Converted-wave seismic exploration: Methods: Geophysics, 67, 1348-1363.

— , 2003, Converted-wave seismic exploration: Applications: Geophysics, 68, $40-57$. 


\section{Appendix A}

\section{Groundroll analysis using}

\section{VSP-type processing}

In traditional seismic data processing, groundroll is considered as noise and has to be removed. In this appendix, I demonstrate a technique that processes groundroll as a zero-offset VSP, which turns groundroll into useful signals.

Figure A.1 shows the physical model used for generating the groundroll data. It includes two materials: Plexiglas and aluminum. For Plexiglas, the P-wave velocity is $2740 \mathrm{~m} / \mathrm{s}$, and the S-wave velocity is $1380 \mathrm{~m} / \mathrm{s}$. For aluminum, the P-wave and S-wave velocities are $6300 \mathrm{~m} / \mathrm{s}$ and $3100 \mathrm{~m} / \mathrm{s}$, respectively.

Figure A.2a shows the geometry of the physical modeling. The source is at $-380 \mathrm{~m}$ from the fault and the first receiver is at $-260 \mathrm{~m}$. The total number of 


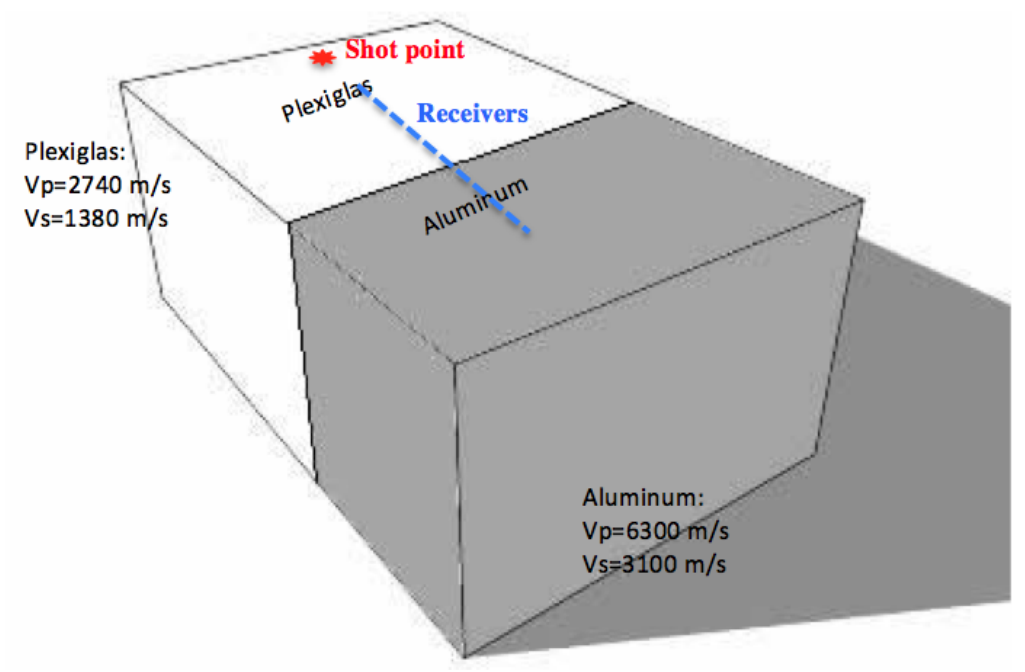

Figure A.1: Physical model that includes two materials: Plexiglas and aluminum.

receivers is 100 with an interval of $5 \mathrm{~m}$. I rebuild the geometry as if it was a zerooffset VSP survey, and Figure A.2b shows the virtual VSP geometry.

\section{A.1 Workflow of groundroll processing}

After transferring the physical modeling data into a virtual zero-offset VSP data, I design a new workflow (see Figure A.3) for processing the groundroll based on routine VSP workflow.

\section{A.1.1 Raw data analysis}

Figure A.4a shows the raw groundroll data after being transferred to VSP geometry from surface geometry. The receiver depth ranges from $120 \mathrm{~m}$ to $620 \mathrm{~m}$. The red 


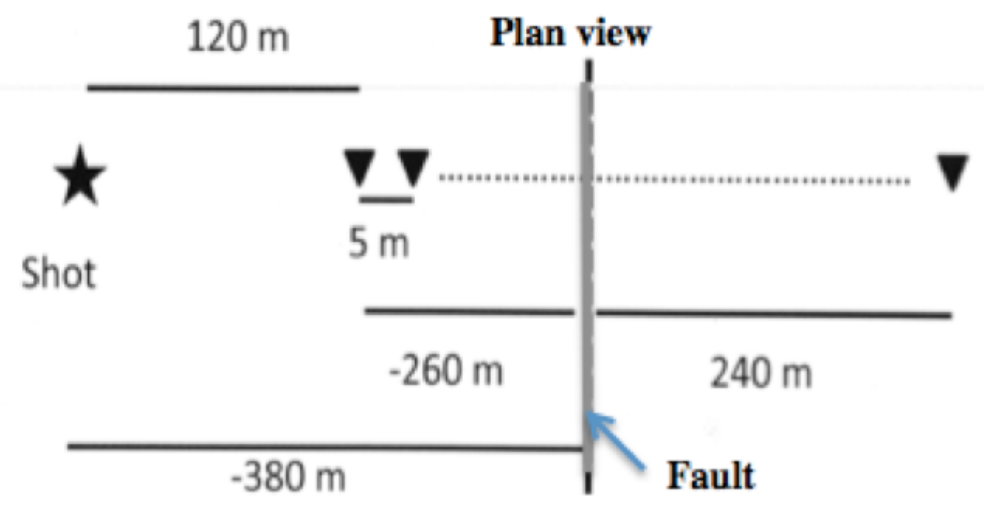

(a)

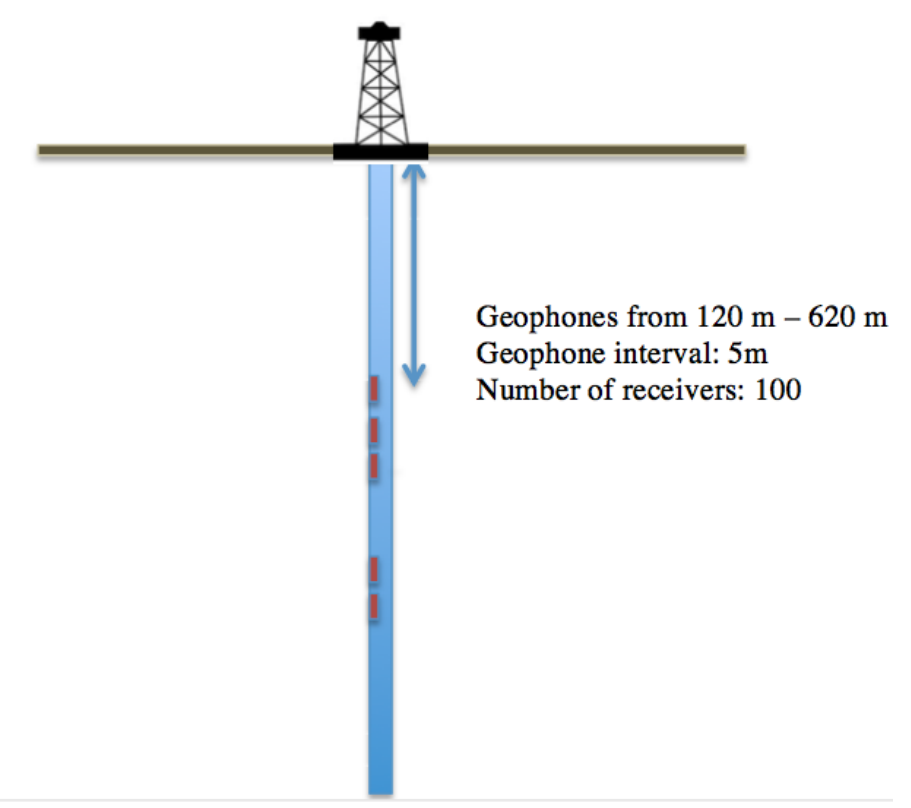

(b)

Figure A.2: (a) Surface geometry used for modeling the groundroll. (b) Virtual zero-offset VSP geometry transferred from the actual surface geometry. 


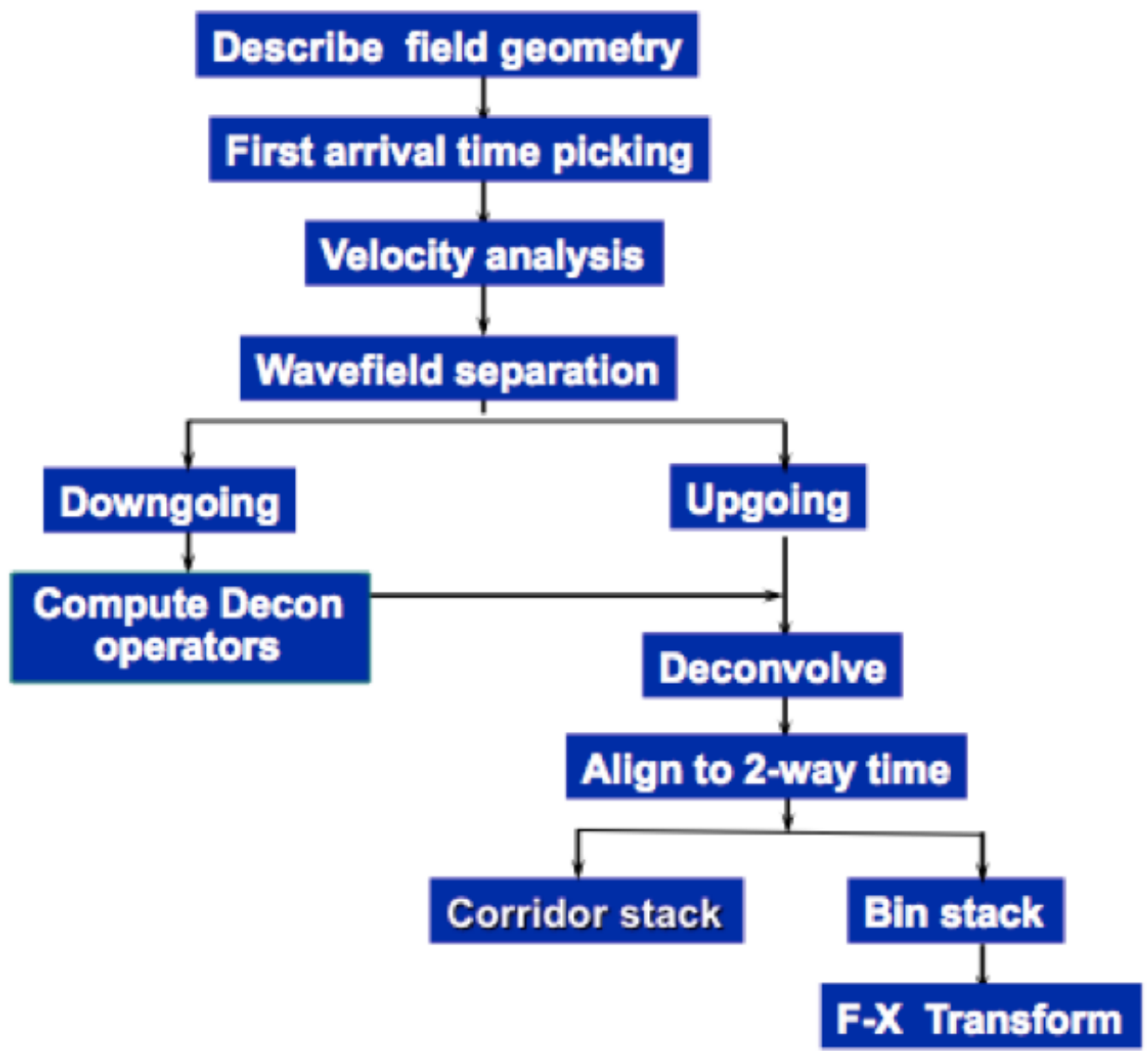

Figure A.3: Workflow of processing groundroll as a virtual zero-offset VSP.

arrows show the strong upgoing and downgoing groundroll, and the green arrow shows the downgoing $\mathrm{P}$-wave.

\section{A.1.2 Traveltime picking and velocity analysis}

Figure A.4b shows the picked first break times of groundroll (red line). The small box on the bottom right is the zoom view.

Figure A.5 shows the average velocity and the interval velocity of the groundroll. 


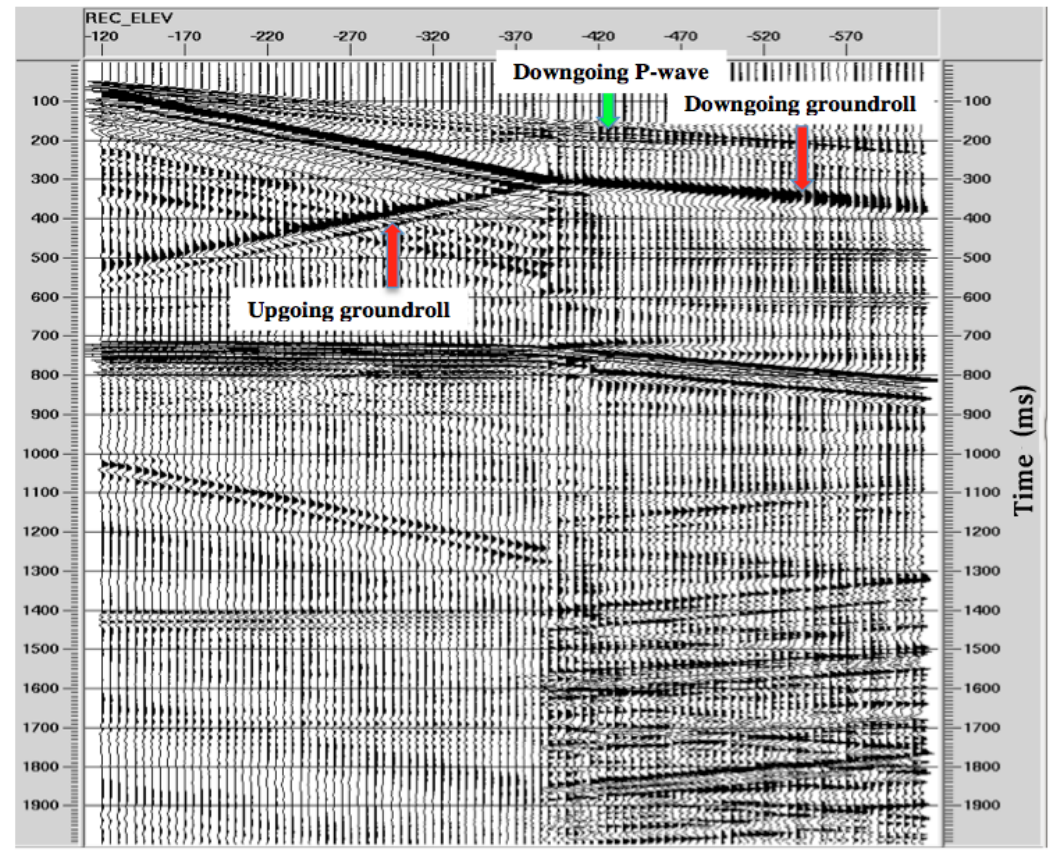

(a)

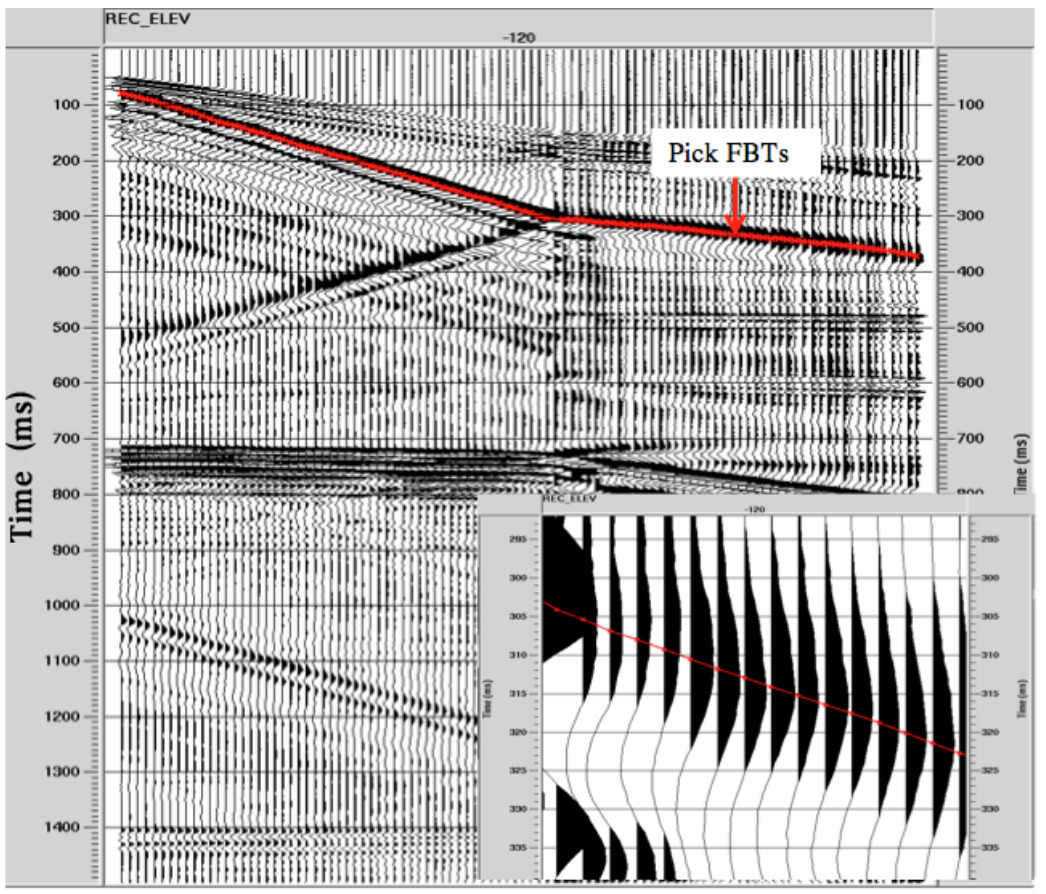

(b)

Figure A.4: (a) Raw groundroll data transferred to virtual zero-offset VSP data. (b) Picked first break times of groundroll and a zoom view of it. 
The estimated interval velocity is around $1200 \mathrm{~m} / \mathrm{s}$ for Plexiglas, which is close to the true modeling velocity $(1380 \mathrm{~m} / \mathrm{s})$. For the aluminum, the estimated velocity is around $3300 \mathrm{~m} / \mathrm{s}$, which is also close to the true one $(3100 \mathrm{~m} / \mathrm{s})$.

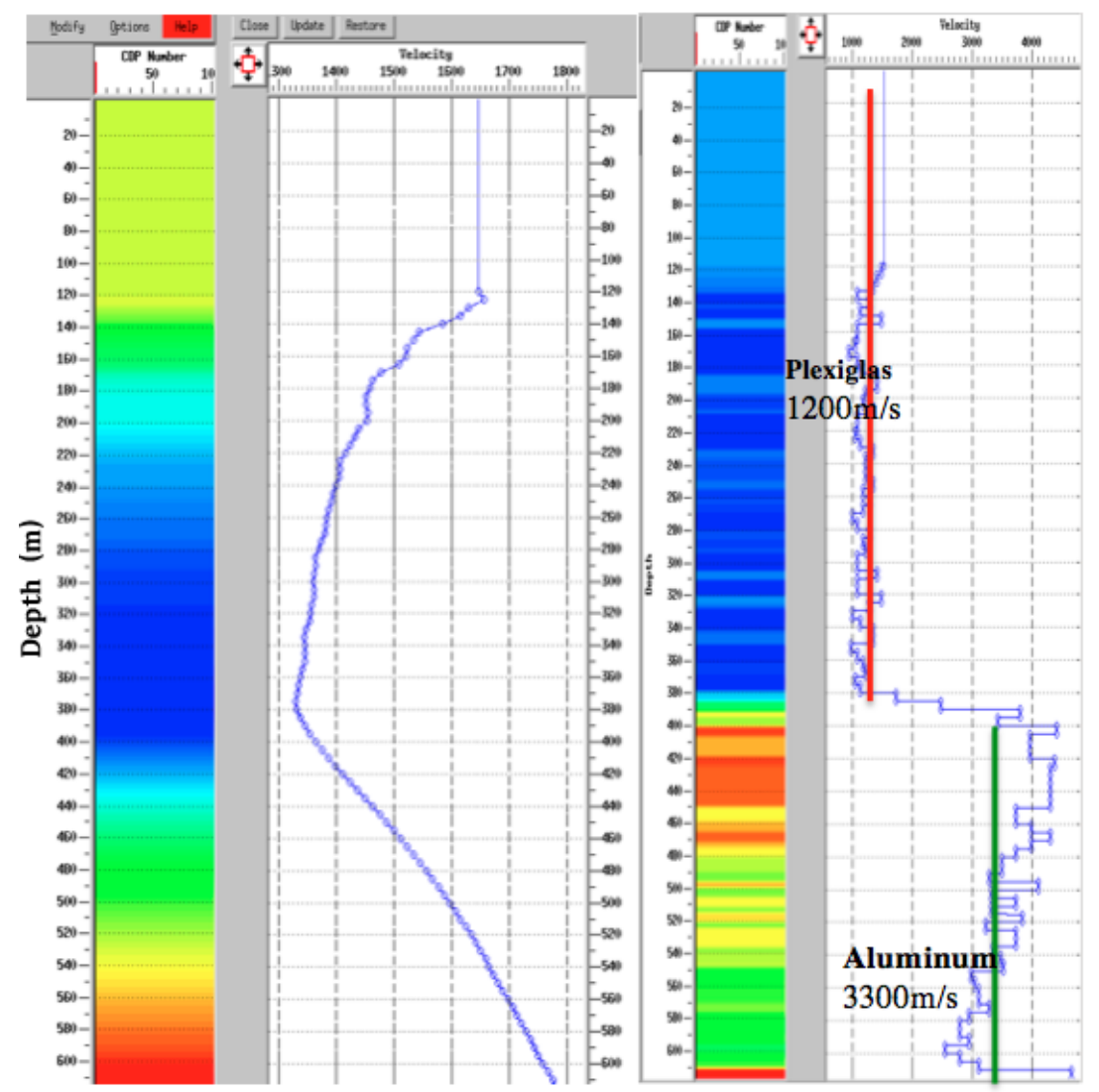

Figure A.5: Average velocity (left) versus interval velocity (right).

\section{A.1.3 Wavefield separation}

Both median filter and FK filter are tested for wavefield separation. Figure A.6 shows the flattened downgoing and upgoing waves that are separated by FK filtering. Figure A.7 shows the separated waves by using median filter. Figure A.7(a) displays 
the raw data that contains both downgoing and upgoing waves. Figure A.7(b) shows the flattened data. Figures A.7(c) and A.7(d) are the separated downgoing and upgoing waves, respectively.

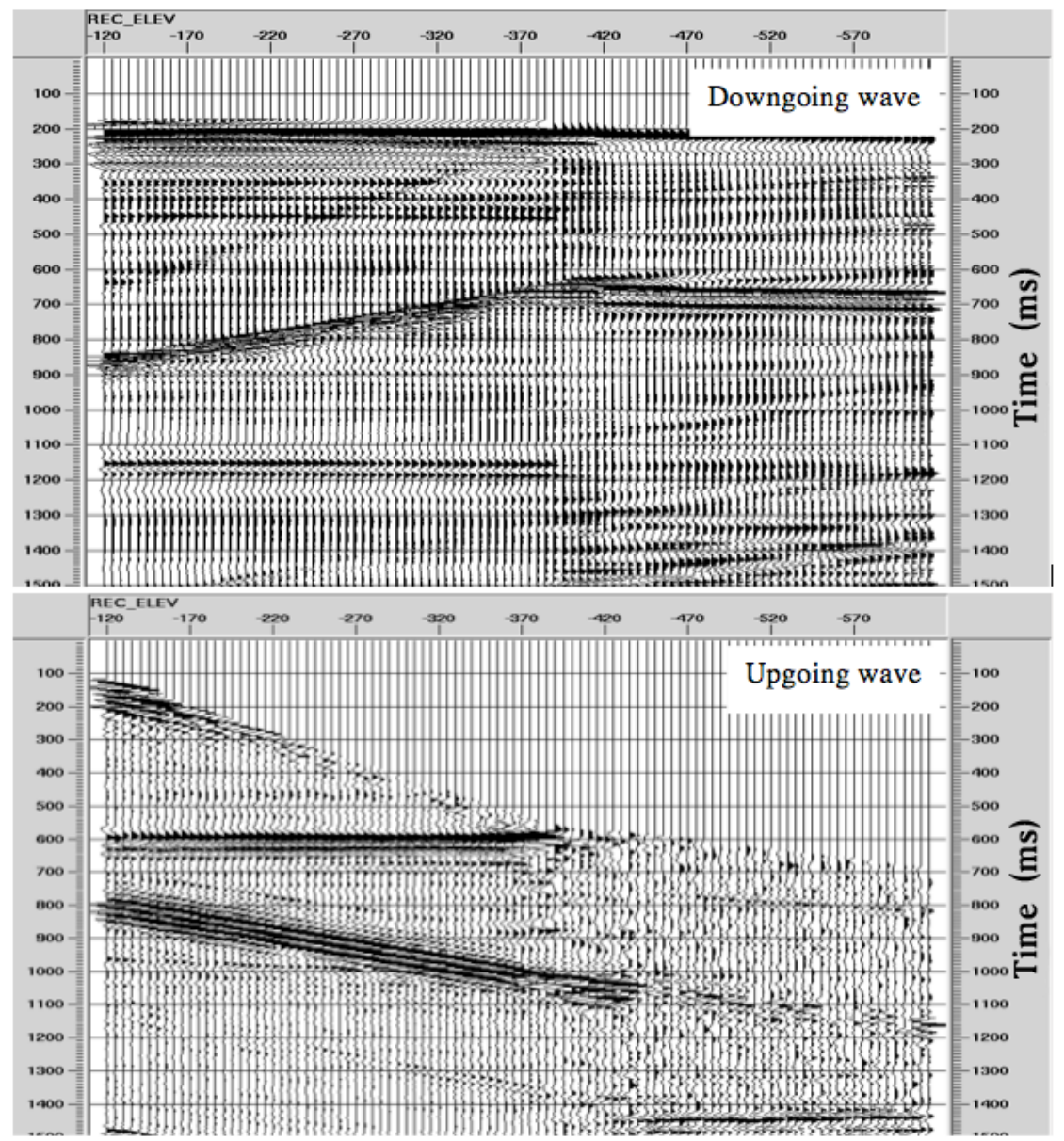

Figure A.6: Downgoing and upgoing waves separated by FK filtering.

\section{A.1.4 Deconvolution}

First, a deconvolution operator is designed by analyzing the downgoing wave. It is then used to shape the downgoing wave. Figure A.8a shows the downgoing groundroll 

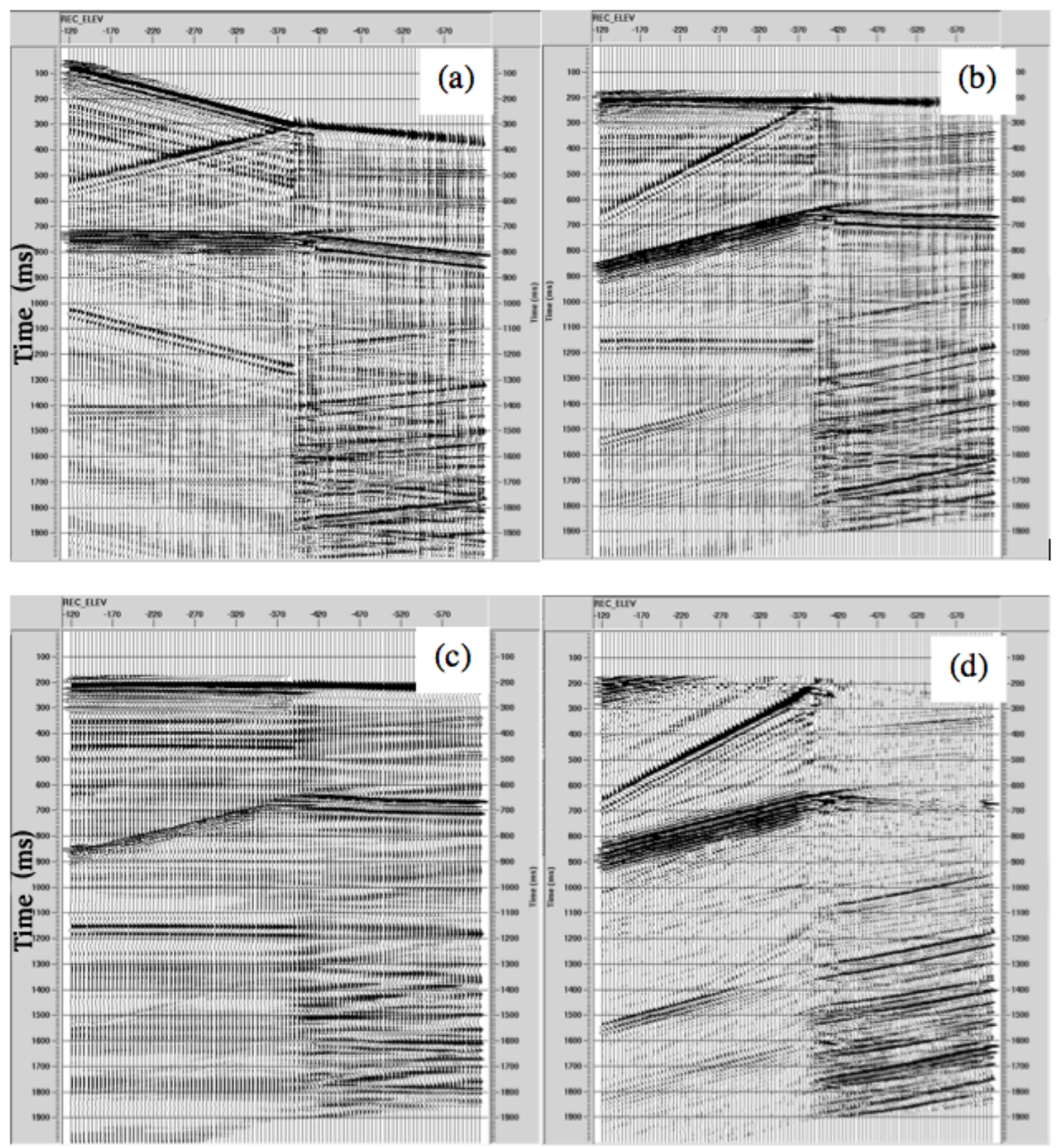

Figure A.7: Separated downgoing and upgoing waves via the use of median filter. 
before and after deconvolution. The same deconvolution operator is applied to the upgoing wave. Figure A.8b shows the upgoing groundroll before and after deconvolution.

\section{A.1.5 Corridor mute and corridor stack}

After deconvolution, the next step is corridor mute and corridor stack. Figure A.9 shows the results. The input of corridor mute is the deconvolved upgoing groundroll.

\section{A.1.6 Bin stack and F-X transform}

Figure A.10 shows the bin stack (left) and its FX transform (right). I choose ten traces in a bin and stack them into a single trace. The total trace number of traces is 100 , after bin stack the number of traces reduces to 10 . The bin stack is then Fourier transformed into $1 / f-x$ domain.

After corridor stack and bin stack, the groundroll processing as zero-offset VSP is completed. 


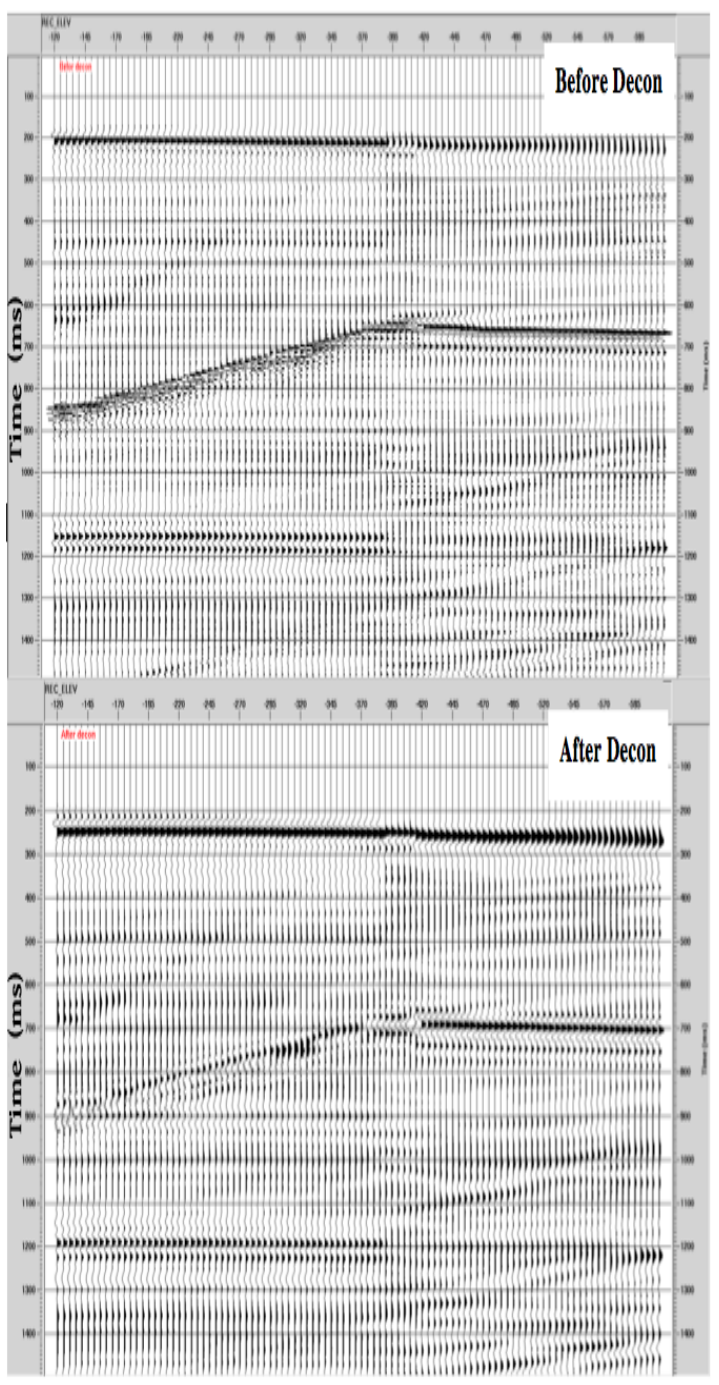

(a)
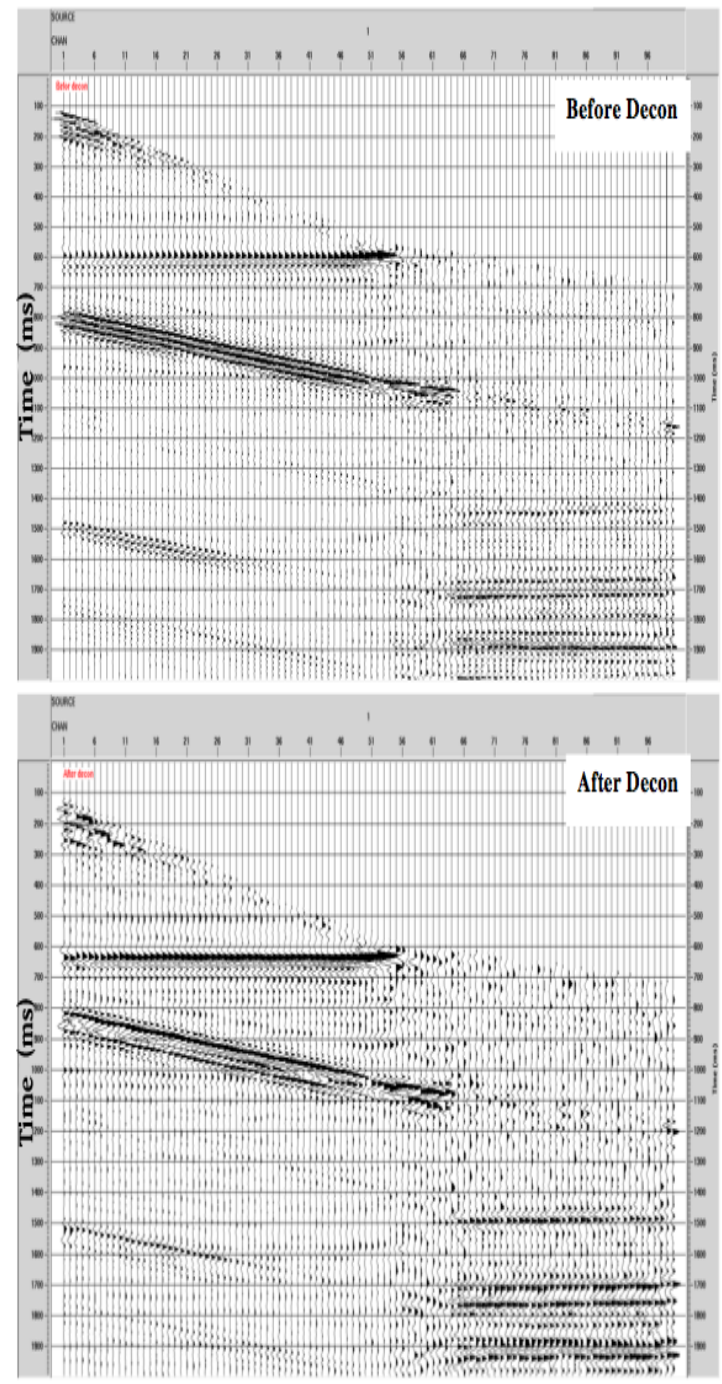

(b)

Figure A.8: (a) Downgoing groundroll before and after deconvolution. (b) Upgoing groundroll before and after deconvolution. 


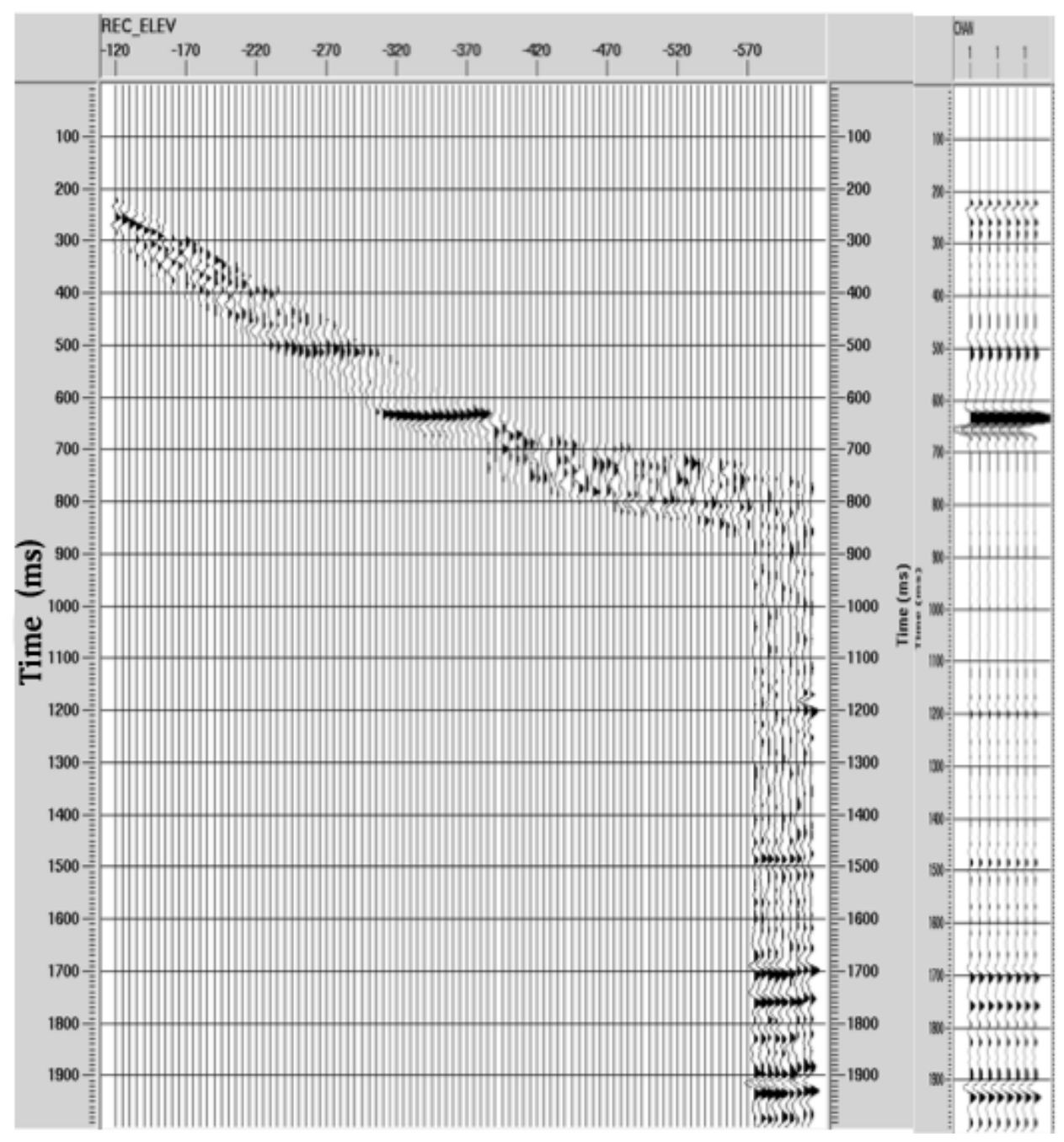

Figure A.9: Corridor mute (left) and corridor stack (right). 


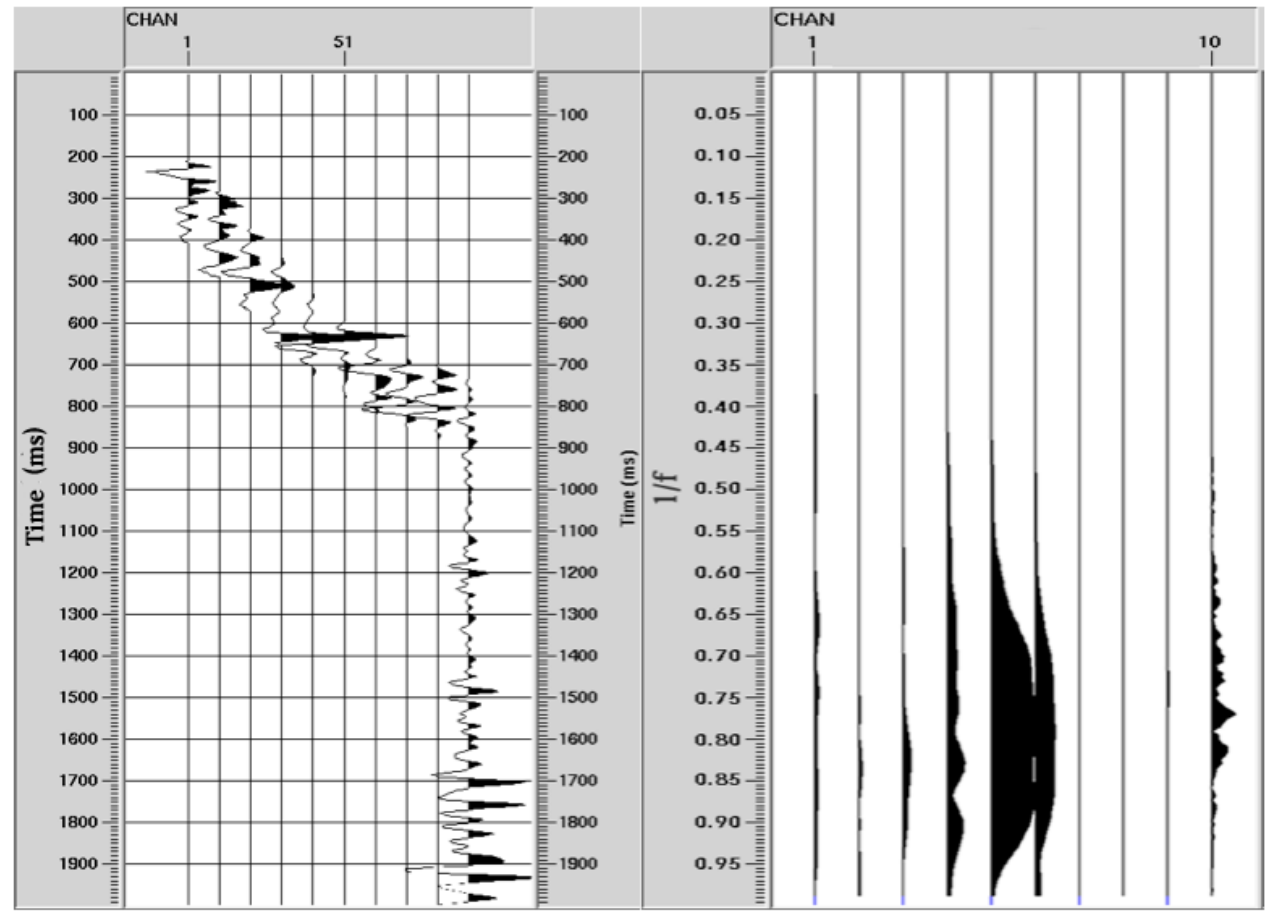

Figure A.10: Bin stack and its FX transform. 